Microelectronics System Design Research Group

School of Electrical and Electronic Engineering

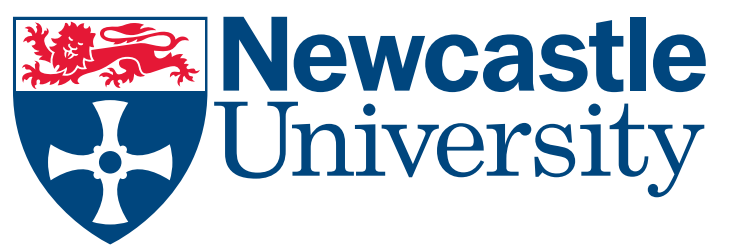

\title{
Redressing Timing Issues for Speed-Independent Circuits in Deep Sub-micron Age
}

\author{
Yu Li
}

Technical Report Series

NCL-EEE-MSD-TR-2012-180

July 2012 
Contact: yu.li1@ncl.ac.uk

NCL-EEE-MSD-TR-2012-180

Copyright (C) 2012 Newcastle University

Microelectronics System Design Research Group

School of Electrical and Electronic Engineering

Merz Court

Newcastle University

Newcastle upon Tyne, NE1 7RU, UK

http: //async.org.uk/ 


\title{
Redressing Timing Issues for Speed-Independent Circuits in Deep Sub-micron Age
}

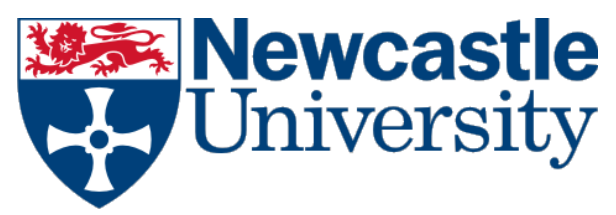

\author{
$\mathrm{Yu} \mathrm{Li}$ \\ Faculty of Science, Agriculture and Engineering \\ Newcastle University
}

A thesis submitted for the degree of

Doctor of Philosophy

2012 July 
1. Reviewer:

2. Reviewer:

Day of the defence:

Signature from head of $\mathrm{PhD}$ committee: 


\begin{abstract}
With continued advancement in semiconductor manufacturing technologies, process variations become more and more severe. These variations not only impair circuit performance but may also cause potential hazards in integrated circuits (IC). Asynchronous IC design, which does not rely on the use of an explicit clock, is more robust to process variations compared to synchronous design and is suggested to be a promising design approach in deep-submicron age, especially for low-power or harsh environment applications.

However, the correctness of asynchronous circuits is also becoming challenged by the shrinking technology. The increased wire delays compared to gate delays and threshold variations could bring glitches into the circuit.

This work proposes a method to generate a set of sufficient timing constraints for a given speed-independent circuit to work correctly when the isochronic fork timing assumption is lifted into a weaker timing assumption. The complexity of the entire process is polynomial to the number of gates. The generated timing constraints are relative orderings between the transition events at the input of each gate and the circuit is guaranteed to work correctly by fulfilling these constraints under the timing assumption.

The benchmarks show that both the number of total constraints and the constraints that are only needed to eliminate strong adversary paths are reduced by around $40 \%$ compared to those suggested in the current literature, thus claiming the weakest formally proved conditions.
\end{abstract}




\section{Acknowledgements}

I would like to express my gratitude to my supervisor, Prof. Alex Yakovlev, for his tremendous help during my Ph.D study. He gave me freedom when new ideas entered my mind and guided me to the right way when I was frustrated. His rigorous attitude to research and diligence to work gave me great encouragement not only in my Ph.D study but also in my future career.

I would like to thank Dr. Terrence Mak for his education on research in both technique aspect and non-technique aspect.

I would like to thank Dr. Fei Xia, Dr. Andrey Moknov and Dr. Danil Sokolov for reading through my thesis and helping me with my English.

I would like to thank Chao Jin and Liuyang Li for their inspiring discussion.

I would like to thank my whole group for their help on my life and for the joy they brought to me.

Especially, I would like to give thanks to my parents. For the incomputable hardships they conquered when bring me up. 


\section{Contents}

List of Figures vi vi vis

List of Tables $x$

1 Introduction 1

1.1 Synchronous and asynchronous circuits . . . . . . . . . . . . 1

1.2 The data path and control path ............. 6

1.3 Significance of the thesis . . . . . . . . . . . . 7

1.4 Organization of thesis . . . . . . . . . . . . . . 9

1.5 Publications . . . . . . . . . . . . . . . . . 10

2 Background 11

2.1 Gate . . . . . . . . . . . . . . . . . 11

2.2 Delay models and types . . . . . . . . . . . . . . . 13

2.3 Signal and Circuit . . . . . . . . . . . . . . . . 14

2.4 Operation modes . . . . . . . . . . . . . . . . 15

2.5 Asynchronous control circuits . . . . . . . . . . . . . . . . 16

2.5.1 Asynchronous design paradigms . . . . . . . . . . 16

2.6 Discussions on the delay model of SI circuits _ . . . . . . . . . 20

3 Speed-independent Circuits 23

3.1 Why SI design . . . . . . . . . . . . . . . . . . . . . . 23 


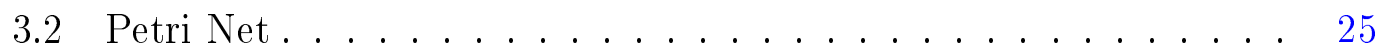

3.3 Signal Transition Graph . . . . . . . . . . . . . . . . . 28

3.4 State Graph . . . . . . . . . . . . . . . . . 30

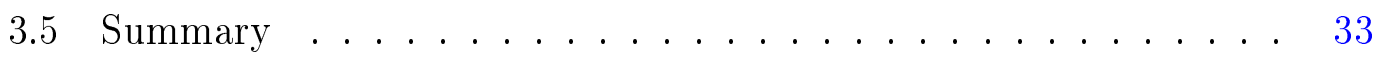

4 Timing Issues in SI Circuits $\quad 34$

4.1 Timing assumptions in SI circuits . . . . . . . . . . . . . . 34

4.2 Existing research on isochronic fork reliability . . . . . . . . 35

4.2 .1 Input negations . . . . . . . . . . . . . 36

4.2.2 Threshold variations . . . . . . . . . . . . . . 37

4.2 .3 Buffer insertion . . . . . . . . . . . . . . . . . . 39

4.3 Recent research on isochronic fork relaxation . . . . . . . . . . . . 41

4.4 Summary . . . . . . . . . . . . . . . . . . 46

5 Hazard checking method 48

5.1 Introduction . . . . . . . . . . . . . . . . . 49

5.1 .1 Overall flow . . . . . . . . . . . . . . 50

5.2 Deriving the Local STG . . . . . . . . . . . . . . . . 52

5.2.1 Decomposition of a free-choice STG into MGs . . . . . . 53

5.2.2 Projecting MG components on operator signals . . . . . 55

5.3 Timing ordering relaxation . . . . . . . . . . . . . 56

5.3.1 Classification of arcs in the local STG . . . . . . . . 58

5.3 .2 Arc relaxation algorithm . . . . . . . . . . . 59

5.3.3 Removing redundant arcs . . . . . . . . . . 68

5.4 Local STG relaxation and hazard criterion . . . . . . . . . 73

5.4.1 Four relaxation cases . . . . . . . . . . . . 73 
5.5 Optimal relaxation order f. . . . . . . . . . . . . 81

5.6 Top level algorithm . . . . . . . . . . . . . . . . . 84

5.6 .1 Complexity analysis ................ 85

5.6.2 Proof of correctness . . . . . . . . . . . 86

5.7 Delay padding to fulfill timing constraints . . . . . . . . . 88

5.8 Summary . . . . . . . . . . . . . . . . . . 90

6 OR-causality Decomposition $\quad 91$

6.1 OR-causality ................................ 91

6.1.1 OR-causality in relaxation case $2 \ldots \ldots . \ldots 92$

6.1.2 OR-causality in relaxation case $3 \ldots \ldots$. . . . . . . 97

6.2 Decomposition of OR-causality _. . . . . . . . . . 100

6.2.1 Generating the solution group . . . . . . . . 106

6.2.2 Decomposition according to the solution group . . . . . . 110

6.3 Summary . . . . . . . . . . . . . . . . . . . . 117

$\begin{array}{llr}7 & \text { Results } & 119\end{array}$

7.1 Design example . . . . . . . . . . . . . . . . . . . . . . . . 119

7.2 Simulation and Analysis . . . . . . . . . . . . . . . . 124

7.3 Benchmarks . . . . . . . . . . . . . . . . . . . . 128

7.3.1 Description of the Tool . . . . . . . . . . . . . 128

7.3 .2 Results. . . . . . . . . . . . . . . . 131

7.4 Summary . . . . . . . . . . . . . . . . . 134

8 Conclusion and Future Work 135

8.1 Conclusion . . . . . . . . . . . . . . . . 135

8.2 Future work . . . . . . . . . . . . . . . . . 137 
8.2.1 Non-free-choice place . . . . . . . . . . . . . . . 137

8.2 .2 Not pure SI circuits . . . . . . . . . . . . . . . . . 138

$\begin{array}{lr}\text { References } & 141\end{array}$

$\begin{array}{lr}\text { Index } & 152\end{array}$ 


\section{List of Figures}

1.1 A synchronous circuit and the equivalent asynchronous circuit . . 2

2.1 A logic gate . . . . . . . . . . . . . . . . 12

2.2 Pure delay and inertial delay . . . . . . . . . . . . . . . . 14

2.3 The Huffman style asynchronous circuit and the Muller style asynchronous circuit . . . . . . . . . . . . . . . . 18

2.4 A 2-input C-element . . . . . . . . . . . . . . . . . . 19

2.5 Glitches with respect to delay models . . . . . . . . . . . . 21

3.1 A PN example. . . . . . . . . . . . . . . . 26

3.2 PN properties . . . . . . . . . . . . . . . . . . 28

3.3 An SI circuit with its $S T G_{\text {spec }}$ and $S T G_{i m p} \ldots \ldots . \ldots 30$

3.4 An STG with its $\mathrm{SG} \ldots \ldots \ldots 32$

4.1 Glitches caused by inverter delay . . . . . . . . . . . . . . 36

4.2 Glitch caused by threshold variation . . . . . . . . . . . . . 38

4.3 Timing difference caused by buffer insertion . . . . . . . . . . . . 40 
4.4 (a) An SI circuit, (b) the STG corresponding to (a), (c) The SI circuit with all wires explicitly denoted by signals and (d) the STG corresponding to (c) when the isochronic fork assumption is removed 43

4.5 Intra operator fork timing assumption . . . . . . . . . . . . . 44

4.6 A counter-example . . . . . . . . . . . . . . . . . 44

5.1 The entire flow of this work . . . . . . . . . 50

5.2 A live and safe free-choice PN (a), and its MG components (b)-(d) 54

5.3 Projection of an STG segment on $X$ and $t \notin X \ldots \ldots 6$

5.4 An $\overline{S R}$-latch with its local STG . . . . . . . . . . . . . 59

5.5 Demonstration of a type $(4)$ arc . . . . . . . . . . . 60

5.6 relaxation of arc $x * \Rightarrow y *$ in the most general case . . . . . . . 61

5.7 un-safeness caused by relaxation . . . . . . . . . . . . . 63

5.8 possible unsafe places after relaxation . . . . . . . . . . . 63

5.9 If two cycles have a common vertex $l \ldots \ldots$. . . . . . . 64

5.10 Shape of MG that will have an unsafe place $<x *, k *>, k * \in y *^{\triangleright}$ after relaxing $x * \Rightarrow y * \ldots \ldots \ldots \ldots 6$

5.11 Shape of the MG that will have an unsafe place $<j *, y *>, j * \in^{\triangleleft}$ $x *$ after relaxing $x * \Rightarrow y * \ldots \ldots \ldots 6$

5.12 Gate $o$ has a redundant literal $p \ldots \ldots$. . . . . . . . 67

5.13 redundant arcs due to the relaxation . . . . . . . . . . 68

5.14 Property of shortcut places . . . . . . . . . . . . . . . . 70

5.15 Check for shortcut places using Dijkstra's algorithm . . . . . . . . 72 
5.16 Illustration of timing conformance. (a) the gate and (b)-(d) local STGs and the corresponding SGs . . . . . . . . . . . 74

5.17 Relaxation case $1 \ldots \ldots 75$

5.18 Relaxation case $2 \ldots \ldots \ldots 76$

5.19 Relaxation case $3 \ldots \ldots 77$

5.20 Relaxation case $4 \ldots \ldots$. . . . . . . . . . . . . . . 78

5.21 Arc modification in case 2. (a) without OR-causality and (b) with

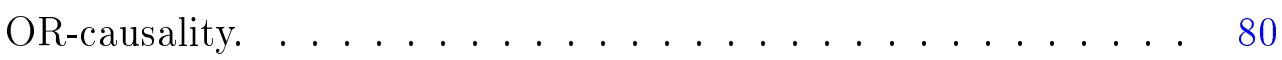

5.22 Preventing the gate entering hazardous state $s \ldots \ldots 82$

5.23 Different timing constraints due to different relaxation ordering . 83

5.24 Calculate the weight of an arc from $S T G_{i m p} \ldots \ldots$. . . . . 84

5.25 Padding positions . . . . . . . . . . . . . . . . 89

6.1 OR-causality in relaxation case 2. (a) the gate and (b)-(f)relaxation

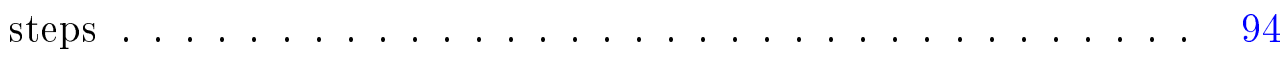

6.2 Candidate clause and transition for OR-causality in relaxation case 2. (a) the gate, (b) and (c) two different STG segments . . . . . 96

6.3 OR-causality in relaxation case 3. (a) the gate, (b) and (c) two different STG segments . . . . . . . . . . . . . . . . . . . 98

6.4 Candidate clause and transition for OR-causality in relaxation case 3. (a) the gate, (b) and (c) two different STG segments . . . . . . 100

6.5 OR-causality decomposition example. (a) the gate, (b) STG segment before decomposition and (c)-(g)resulting subSTG segments 102 
6.6 An OR-causality relation in case 2. (a) the gate and (b) its local STG segment . . . . . . . . . . . . . . . . . . . . . 114

6.7 Decomposition results for the OR-causality relation in Figure 6.6.

(a)-(e) resulting subSTG segments . . . . . . . . . . . . . 115

6.8 An OR-causality relation in case 3. (a) the gate and (b) its local STG segment . . . . . . . . . . . . . . . 116

6.9 Decomposition results for the OR-causality relation in Figure 6.8.

(a)-(d) resulting subSTG segments . . . . . . . . . . . . 117

7.1 The block diagram and STG specification of FIFO . . . . . . . 120

7.2 The implementation STG and circuit diagram of FIFO . . . . . 121

7.3 The STG relaxation procedure of the gate_0 . . . . . . . . 122

7.4 The current starved delays. (a) controlled delay for rising transition and (b) controlled delay for falling transition . . . . . . . 125

7.5 The trend of error rate as the technology shrinks . . . . . . . . . 126

7.6 The trend of error rate as the scale increases . . . . . . . . . . . 127

7.7 The delay penalty . . . . . . . . . . . . . . . . . . . . . 128

8.1 A non-free choice STG and its equivalent free-choice STG . . . . 139 


\section{List of Tables}

7.1 List of timing constraints . . . . . . . . . . . . . . . 124

7.2 Comparison of the timing constraints . . . . . . . . . 133 


\section{Chapter 1}

\section{Introduction}

As the process shrinks, the traditional synchronous design faces great challenges. Meanwhile, the asynchronous design exhibits advantages in many important aspects, such as the tolerance to process variation and reduced power consumption. This chapter briefly compares the advantages and disadvantages of synchronous and asynchronous designs to illustrate why the asynchronous design suggests a promising design approach in the near future.

\subsection{Synchronous and asynchronous circuits}

Digital circuits can be partitioned into combinational logic, in which the output signals depend only on the current input signals, and sequential logic, in which the output depends both on current input and the past history of inputs(state of the circuit). Sequential logic is combinational logic with storage components (latches). 


\subsection{Synchronous and asynchronous circuits}

In synchronous circuits all latches change according to the same periodic global signal, called the clock. Inputs to latches must be stable before clock events arrive and all latches change simultaneously when the clock events arrive. Clock is used to synchronize the data transferring between combinational logic blocks and filter out unexpected transient events (called glitches) before the circuit becomes stable. In contrast, asynchronous circuits do not use the clock. Operation on one latch is triggered by the events coming from its controller, which communicates with other controllers by handshake protocols.

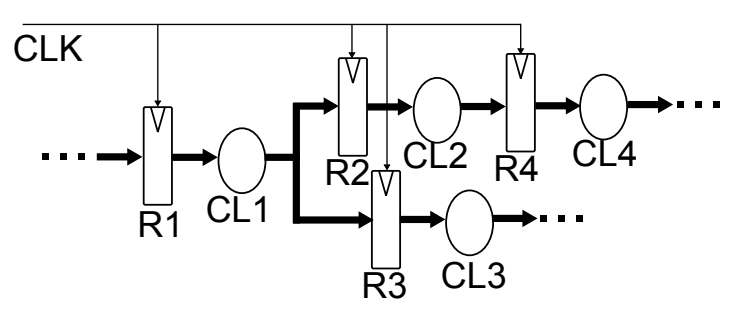

(a)

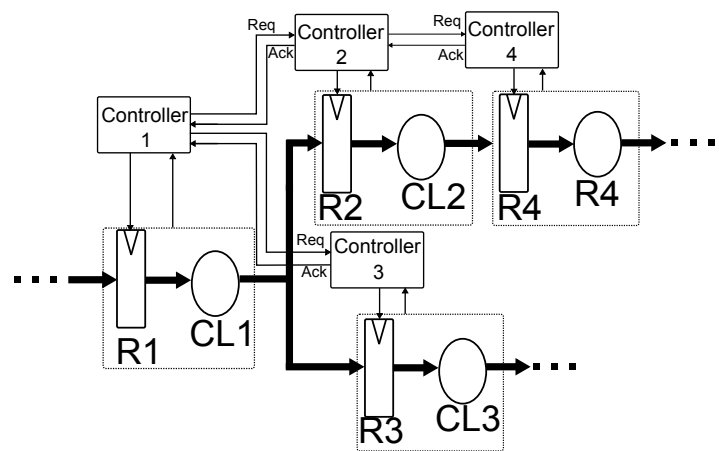

(b)

Figure 1.1: A synchronous circuit and the equivalent asynchronous circuit -

The schematic diagram of a synchronous circuit is shown in the diagram (a) in Figure 1.1 with the schematic diagram of the equivalent asynchronous circuit shown in diagram (b). In the synchronous circuit, four registers (R1-R4) are controlled by the clock signal (CLK). The clock cycle period must be greater than the worst delay of all combinational logic blocks in the circuit and all combinational logic blocks (CL1-CL4) will be synchronized by the end of each clock cycle. However, in the asynchronous circuit, the clock is not used. The transformations 


\subsection{Synchronous and asynchronous circuits}

on the data are synchronized by the handshake signals. Request (Req) will be sent to the controller of the sink of the data from the source controller when the data from the source is ready and the acknowledge (Ack) will be sent back to indicate the completion of the operation.

Thanks to the Moore's law[1], the complexity of the integrated circuit doubles every 18 months. Nowadays, a single chip could contain more than one billion transistors. Certain problems, which were not quite severe in the last few decades, are becoming critical today or will be critical in the near future. The synchronous design, which introduces a global clock to mask glitches and divide the combinational and sequential logics, has been the mainstream in the digital integrated circuit community. However, the weakness of synchronous design is exposed when the semiconductor technology shrinks.

Performance and power:

It is very costly to distribute a global clock signal on a multi-billion transistor chip. Clock signal skews along the large distribution tree. As the number of transistors increases and the delay of transistor decreases, the clock skew problem becomes more and more severe. Additional area or clock magnitude needs to be sacrificed in order to guarantee the correctness of the circuit. In addition, the power consumption related to the distribution clock signal consumes the largest proportion in a synchronous circuit. Currently, up to $40 \%$ of total power is consumed by the clock distribution network [2] and this situation becomes worse as the complexity and the frequency of the circuit grows [3].

Meanwhile, although the clock could prevent glitches from causing errors in synchronous circuits, glitches do dissipate energy. Glitches are useless transi- 


\subsection{Synchronous and asynchronous circuits}

tions, which could propagate in the combinational logics and cause additional transitions. As reported in [4], the power dissipation related to glitches in CMOS technology consumes up to $15 \%$ of total power.

As the feature size of technology shrinks, the process variations become a new important factor that influences the performance of digital circuits. In order to get an acceptable yield, synchronous design needs to set its clock period conservatively.

Unlike the synchronous design in which glitches could be filtered out by the clock, asynchronous circuits are usually vulnerable to glitches. The handshake protocols cannot distinguish between a real transition and a glitch. Any glitch could be considered by some logic as a premature transition and causes hazards. Designers of asynchronous circuits usually put quite a lot of effort to avoid dangerous races in the circuit. This always results in that asynchronous design consumes more area and efforts compared to the synchronous design. Due to the simplicity, synchronous design dominates the integrated circuit market during past decades. But, as the mobile electronics devices become the mainstream of the consumer electronics, the performance and energy dissipation become the two most concerned aspects for industry designs. In contrast, the area now becomes a less concerned aspect. All those above indicate that asynchronous circuits suggest a promising design paradigm, which offers a high performance and low power consumption solution in the coming decades for both the technical and commercial reasons.

\section{Modularity :}

The circuit design trends to compose a powerful system by many small com- 


\subsection{Synchronous and asynchronous circuits}

putational modules or intellectual property (IP) cores, which communicate with each other through protocols to achieve higher energy efficiency. Directly connecting multiple synchronous modules together is very difficult if not impossible. The asynchronous handshake suggests a promising approach to be the interface protocol to connect sub-modules. As expected by ITRS [5], by 2022, up to $45 \%$ signals of a design will be driven by handshake.

Without doubt, asynchronous circuits will attract more designers' attention in the coming decades. The inherent request and acknowledgement mechanism can avoid the clock skew and distribution problem. Also, this mechanism will automatically shut down unused parts in a circuit and avoid generating the unneeded transitions and thus reduces the power consumption. Different modules could be easily connected together under protocol based scheme. The strict glitch-free requirement and the conservative delay assumption makes asynchronous circuits have much stronger variation tolerance ability compared to the synchronous counterpart.

However, the asynchronous design paradigm also meets challenges.

Without the clock to filter out glitches, asynchronous circuits suffer from race hazards[6], which means that circuits might exhibit glitches or even go into the wrong state depending on differences in delay of elements in circuits. In order to avoid race hazards, the synthesis of asynchronous circuits needs to fulfill additional requirements. These requirements make asynchronous circuits more difficult to design compared with synchronous circuits. Also, the automatic synthesis of asynchronous circuits usually needs to explore the entire state space to fulfill the hazard-free requirement and optimize the logic. Asynchronous circuits 
usually exhibit highly concurrency among events. The computation complexity is in the order of $O\left(2^{n}\right)$, with respect to the number of signals $(n)$ in the circuit. This makes asynchronous circuits hard to design even in moderate size.

Asynchronous circuit design paradigm does not have an entire design flow support. The EDA tool support for asynchronous design is poor, not only because there is no uniformed design paradigm but also the real difficulty behind this matter. Also, the asynchronous circuit is not only hard to design but also to test.

Due to the problems mentioned above, designing asynchronous circuit always requires experienced designers. Therefore, the time cost for designing asynchronous circuit is usually much greater than for designing a synchronous one.

Nowadays, the semiconductor technology goes into deep sub-micron age and the design trends to many-core, low power, environment variation robust and process variation tolerance applications. These requirements just meet the characteristics of asynchronous circuits.

\subsection{The data path and control path}

A circuit is typically partitioned into two main parts, the data path and the control path. The data path usually includes the units to process the data, e.g. adders and the units for storage and communication e.g. registers. The control path usually provides signals that control the data path to work properly, e.g. operation codes and the clock signal. In Figure 1.1, the control of the two circuits includes the clock (CLK) and asynchronous controllers; the datapath includes registers and the combinational logic. This thesis focuses on controllers in asyn- 
chronous circuits (like the controllers 1-4 in diagram (b) in Figure 1.1. Circuits discussed in this thesis refer to the control circuits if not specified otherwise.

\subsection{Significance of the thesis}

Among all asynchronous design paradigms, delay-insensitive circuits, which could tolerate arbitrarily large delay variations on both gates and wires, show the strongest process variation tolerance ability. However, delay-insensitive circuits have been proved to be quite limited that only a very small set of specifications have a delay-insensitive implementation[7]. This also indicates that for almost all useful specifications, the implementations should contain some timing assumptions in them. Speed-independent circuits, which only take the isochronic fork timing assumption, are supposed to be the paradigm that imposes the weakest timing assumption. Speed-independent circuits could work correctly under many harsh situations, e.g. VDD variations and the gate delay variations. However, the other variations like the threshold variation could still cause speed-independent circuits to malfunction.

Speed-independent circuits suggest a good starting point to correctly implement circuit under unprecedented variations. Unlike the design paradigms that introduce the real time information in synthesis, speed-independent circuits only compare the arriving orders of events. They therefore redress only those timing issues needed to guarantee the required orders. This is desirable in the deep submicron age. The delay variations are quite large that estimating the exact time is difficult and unreliable in the deep submicron age. However, orders between 
two events are much easier to predict and fix. Currently, most layout tools does not directly support relative timing constraints. This is because the synchronous design is widely adopted by the industry and synchronous tool is well developed where the numerical delay is used. However, the layout tool for the asynchronous design that supports the relative timing constraints could be developed as the asynchronous design becomes more and more important.

The verification of the timing fulfillment is a very difficult and time consuming task. The time complexity usually reaches the exponential or even double exponential order with respect to the number of signals in a circuit. This is hardly acceptable even for a moderate scale circuit.

This thesis proposes an efficient method to verify and re-synthesize speedindependent circuits. It takes a reasonably weaker timing assumption compared to the isochronic fork timing assumption and then introduces a series of algorithms to verify circuit and generate a set of sufficient timing constraints to guarantee the correctness of the circuit. The generated timing constraints could always be fulfilled.

The main contributions of this thesis are:

1) It corrects some wrong conclusions given by previous researchers about the weakest timing assumption in speed-independent circuits.

2) It introduces a hazard checking criterion for speed-independent circuits when the isochronic fork timing assumption is relaxed.

3) Most importantly, this thesis proposes a method utilizing properties of the speed-independent circuit to do the timing verification in polynomial time. This method divides the entire verification problem into smaller sub-problems and thus 
avoids exploring the full state space.

The limitation of this thesis is that in the point 3) mentioned above, one operation "projecting a Petri Net on a subset of transitions" is needed. However, this is an open question for general Petri Nets. Thus, the input signal transition graph to this technique (one kind of Petri Net) is limited to a free-choice Net, where Hack's algorithm[8] could apply.

\subsection{Organization of thesis}

This thesis is organized as follows:

Chapter 1 briefly introduces the synchronous and asynchronous design and presents the significance of this thesis.

Chapter 2 defines the terms used in asynchronous community and introduces different asynchronous design paradigms.

Chapter 3 introduces the related descriptions and models of speed-independent circuits, which will be used in the following chapters and explains why speedindependent circuits are adopted by this thesis among asynchronous design paradigms.

Chapter 4 investigates the possible situations that could cause failures of the isochronic fork and discusses the technology trends that affect these situations. Also, related research is overviewed in this chapter.

Chapter 5 presents the main method for hazard checking when the fundamental timing assumption of speed-independent circuits is relaxed.

Chapter 6 analyzes one complex problem, the OR-causality, which may occur during the hazard checking process presented in Chapter 5 in detail and proposes 
a technique to solve this problem.

Chapter 7 presents the benchmark results of the method. This chapter compares the tightness of the generated timing constraints with similar research. Also one design example is presented in detail to demonstrate the proposed method and to show the penalty introduced by eliminating the potential hazards.

Chapter 8 concludes the thesis and discusses the possible ways to break the limitations of the proposed method to make it suitable for broader range of specifications.

\subsection{Publications}

The main results of the thesis have been published in the following paper:

- "Relative Timing Applied to Asynchronous Circuit Synthesis and Decomposition" (19th UK Asynchronous Forum)

- "Conditions and Techniques for Correctness of SI/QDI Circuits Under Large Variability" (21st UK Asynchronous Forum)

- "Redressing timing issues for speed-independent circuits in deep submicron age" (DATE'11) 


\section{Chapter 2}

\section{Background}

This chapter gives the definitions of basic elements and concepts of a digital circuit and also introduces popular asynchronous design paradigms.

\section{$2.1 \quad$ Gate}

Gates are basic elements in a circuit. In this thesis, a gate is defined as an $n$ inputs and one output boolean variable. If the inputs contain its output variable, the gate is sequential, otherwise it is combinational. For every gate there is an associated logic function $f$ to compute it.

The definition of logic function in [9] is adopted.

A logic function $f$ with $n$ input variables is a mapping $f:\{0,1\}^{n} \mapsto\{0,1\}$, where $\{0,1\}^{n}$ is a binary vector over its input variables called input state. The set of input states that maps to ' 1 ' is the on-set of $f$, while that maps to ' 0 ' is its off-set. 
A literal is a variable $x$ or its complement $\bar{x}$. A cube $c$ is a set of literals on different variables, which means that literal $x$ cannot appear multiple times and $x$ and $\bar{x}$ cannot appear simultaneously in a cube. A cube $c$ represents the vertexes corresponding to the boolean product of its literals. A cube $c^{\prime}$ is covered by another cube $c^{\prime \prime}$ if $c^{\prime \prime} \subseteq c^{\prime}$, denoted by $c^{\prime} \sqsubseteq c^{\prime \prime}$.

A cube is an implicant of a logic function $f$ if it does not cover any vectors in off-set of $f$. An implicant of $f$ is called a prime implicant if it cannot be covered by any other implicants of $f$. A cover $U$ is a set of cubes, which represents the boolean sum of its cubes. A cover $U$ is an on-set cover of logic function $f$ if each cube in $U$ is an implicant of $f$ and each vertex in $f$ is covered by at least one cube in $U$. A cover $D$ is an off-set cover of logic function $f$ if $D$ is an on-set cover of $\bar{f}$, where $\bar{f}$ is a logic function obtained by exchanging the on-set and off-set of $f$. A cover $U$ is a prime cover of logic function $f$ if all its cubes are prime implicants of $f$. A cube $c$ is redundant in a cover $D$ of logic function $f$, if $D \backslash c$ is still a cover of $f$. A cover $D$ is redundant for $f$ if at least one cube in it is redundant, otherwise it is irredundant. An irredundant prime cover of logic function $f$ is denoted by $f_{\uparrow}$ and an irredundant prime cover of $\bar{f}$ if denoted by $f_{\downarrow}$. Each cube in $f_{\uparrow}$ and $f_{\downarrow}$ is called a clause. The notation $f_{a \uparrow}$ and $f_{a \downarrow}$ is used if $f$ computes gate $a$.

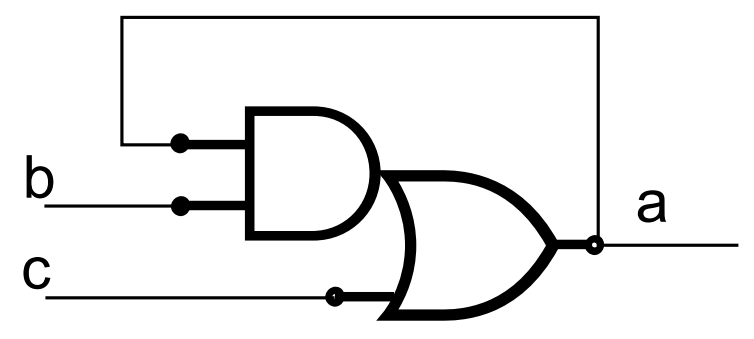

Figure 2.1: A logic gate - 
An example of a gate $a$ is shown in Figure 2.1, the gate $a$ is a sequential gate. Its inputs are $a, b$ and $c . f_{a \uparrow}=a \cdot b+c$ and $f_{a \downarrow}=\bar{a} \cdot \bar{c}+\bar{b} \cdot \bar{c}$.

\section{$2.2 \quad$ Delay models and types}

Delay is an inherent property of all electronic components. For simplicity, in digital circuits, the delays are usually abstracted out of the component and are denoted as separated elements. The original components are then assumed to be instantaneous. The property of delay is depicted by a separated delay element. For different designs and circumstances, different delays are used [6].

A delay element is a pure delay if the delay only shifts every transition for a given magnitude.

A delay element is an inertial delay if the delay not only shifts transitions but also absorbs any pulse that is narrower than the magnitude of the delay.

A delay is unbounded if the magnitude of the delay could be any positive value. A delay is bounded if the interval of the delay magnitude is given.

Figure 2.2 represents the comparison of the pure delay with the inertial delay. The delay magnitudes for these two delays are larger than $t 1$ but smaller than $t 2$.

In speed-independent circuits, every gate has a pure and unbounded delay and each wire does not have any delay. The effect of this assumption is that every gate computes its new output as soon as its inputs change and if there is a transition due to the input change, the transition will be delayed for a given time and then transmitted to its next-level simultaneously. The pure delay assumption is always much safer compared to the inertial delay assumption with respect to 


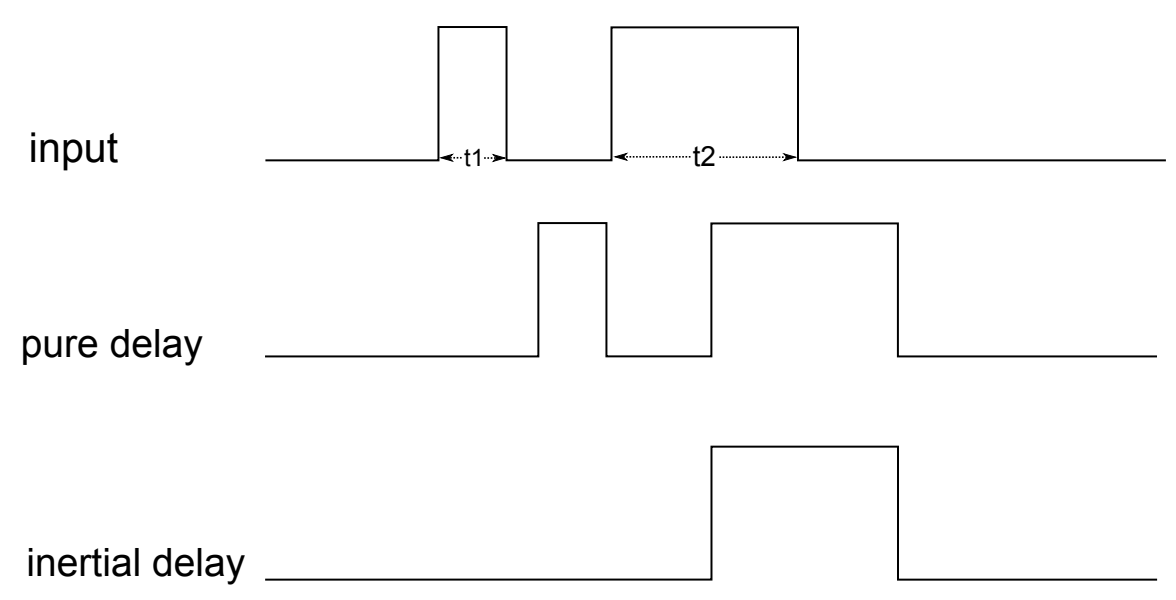

Figure 2.2: Pure delay and inertial delay -

glitch-freedom as will be analyzed in the following section.

\subsection{Signal and Circuit}

Transitions on signals represent the dynamics of a circuit. The set of signals should depict the entire reachable states of a circuit. Here, we define the signals of a circuit (denoted by the set $A$ ) to be the union of the primary input variables and gate variables. For circuits which are in context with their environment $(E N V)$, the signals coming from the primary inputs are denoted by a set $I$, the gate variables which feedback to the $E N V$ are primary outputs denoted by a set $O$ and the gate variables that are not primary outputs are internal signals denoted by a set $R$. For autonomous circuits, we have $I=O=\varnothing$ and $R=A$.

A circuit is defined as a pair $C=(A, \varphi)$, where $A$ is a set of signals and $\varphi$ is a labeling function which labels a wire between each signal $a \in I \cup R$ and each fan-out of $a$. 
In the definition above, only input signals and gate variables are used to describe the dynamics of a circuit, wires will not be shown explicitly. This is because the type of the asynchronous circuit under discussion is the speed-independent circuit, where wires could be considered to have zero delays, the dynamics of wires could be fully represented by the gate behaviors. When the isochronic fork is relaxed (as will be specified in the following chapters), we will still use techniques to avoid introducing signals to wires. The reason behind this is as follows. Firstly, the number of wires is always equal to or more than the number of gates in a circuit, so encoding using wire signals would increase the computational complexity. Secondly, introducing signals to wires will break some properties that are necessary for us to model the behavior of speed-independent circuits (will break the safeness of a $\mathrm{PN})$.

\subsection{Operation modes}

The interface mode defines how a circuit interacts with its environments. There are two classical modes [6] that adopted by different asynchronous design paradigms:

1) The fundamental mode/burst mode, when the circuit is stable, one primary input/one or more primary inputs are allowed to change. The environment cannot change inputs again unless the entire circuit becomes stable.

2) The input-output mode, the environment could change the primary inputs of the circuit as soon as it sees the expected transitions on primary outputs.

The fundamental mode requires that the environment keeps all transitions on primary input signals longer than the maximum delay in the circuit. This 
requires designer to estimate the real delays in a circuit. While input-output mode requires that every signal transition is acknowledged to make sure that when the environment sees the expected transitions on primary outputs, all internal signal transitions have happened.

\subsection{Asynchronous control circuits}

The class of asynchronous circuits is a very broad class. Different researchers often propose quite different design flows, where different specification formalisms, various assumptions, synthesis techniques and manpower are utilized. This section will focus on the introduction of popular asynchronous design methods and their trade-offs. Circuits discussed in this section refer to the control circuits if not specified otherwise. Also, methods referred in this thesis are mainly related to the design of control. The datapath usually contains a large number of signals in high concurrency. Techniques that focus on the control are usually not capable of handling datapath and the synthesized circuits would be inefficient. However, a large body of research exists for datapath circuits as well[10] [11] [12].

\subsubsection{Asynchronous design paradigms}

The Huffman style asynchronous circuits: The Huffman style asynchronous circuits are first proposed in [13], which take the bounded wire and gate delay model and operate under the fundamental mode. The schematic diagram of the Huffman style asynchronous circuits is shown in Figure 2.3 (a). 


\subsection{Asynchronous control circuits}

The specification of the fundamental mode Huffman style asynchronous circuits is often a Huffman flow table which represents an asynchronous finite state machine (ASFM). The circuit consists of primary input signals $(a, b, c$ in Figure 2.3 ), primary output signals $(x, y, z)$, state signals (feedback signals from output to input, $M, N, m, n)$ and the combinational part of the circuit. In the initial state, one input of the circuit is allowed to change. Then the combinational part of the circuit starts to compute the new output value and the next state value. When the environment receives the new output value, it cannot provide a new input transition until the circuit becomes stable after receiving the next state value.

The Huffman style asynchronous circuits, which are quite similar to the synchronous circuits, are easier to design compared to those in other design methods. But this kind of circuits has two limitations. Firstly, the operations of the circuit must follow a strict order that one input must change first followed by the state signals and the output signals. Therefore, concurrency between the input changes and output changes is not allowed (also the finite state machine cannot depict this kind of concurrency). Secondly, delays or other techniques must be used to guarantee that the next state value cannot propagate to the combinational part too early and the environment does not provide a new input transition too early.

The fundamental mode Huffman style asynchronous circuits have the limitation that only one input could change at one time. The early work related to the synthesis of fundamental mode Huffman style asynchronous circuit is presented in [13] [14]. Steven Nowick proposed a burst mode Huffman style asynchronous paradigm in [15], which expanded the concurrency to allow a constrained set of 
input signals (input burst) to change concurrently. However, the burst mode asynchronous circuits still do not allow the concurrencies between input bursts and output bursts and still require the timing constraints in the fundamental mode. The synthesis of the burst mode asynchronous circuits is automated in the tool MINIMALIST[16].

The burst mode design style is further expanded by Yun and Dill into the extended burst mode design style[17][18], which allows an input signal to change concurrently with output signal and allows control flow to depend on the input levels. With these extensions, the burst mode design style covers a wide spectrum of sequential ranging from asynchronous to synchronous. The extended burst mode specification could be synthesized by the 3D synthesis tool[19].

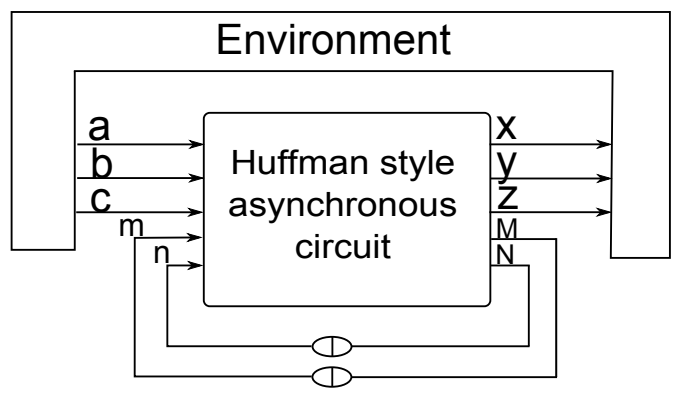

(a)

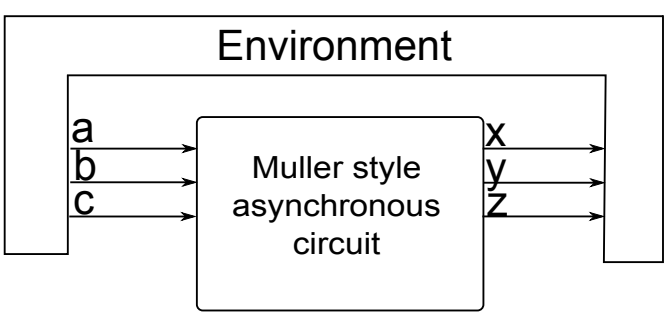

(b)

Figure 2.3: The Huffman style asynchronous circuit and the Muller style asynchronous circuit -

The Muller style asynchronous circuits: Unlike the Huffman style asynchronous circuits, the Muller style circuits do not put so many restrictions on specifications and the environment. The schematic diagram of the Muller style circuit is presented in Figure 2.3 (b). The operation of the circuit is based on the following 
protocol: the environment is allowed to provide new inputs as soon as it sees expected outputs. Also, the specification of Muller style circuits does not constraint the concurrency between signals. The design of input-output mode Muller style asynchronous circuits was introduced in [20] [21].

The Delay-Insensitive (DI) circuits:

The DI circuits are one kind of Muller style asynchronous circuits that work correctly even if every wire and gate has unbounded delay. A very important type of gate in DI circuits is a C-element. The symbol and the truth table of a 2-input C-element is shown in Figure 2.4. A more general definition for C-element is that output changes if and only if all of its inputs change. So, an inverter is also a 1-input C-element. Among all asynchronous design paradigms, only DI circuits do not have any timing assumption. So, DI circuits could tolerate arbitrary delay variations on their components.

But as was proved in [22], the DI circuits are quite limited: if an autonomous DI circuit is built of single output gates, then all gates must be C-elements. Moreover, the C-element itself does not have a DI implementation built of basic gates[23].
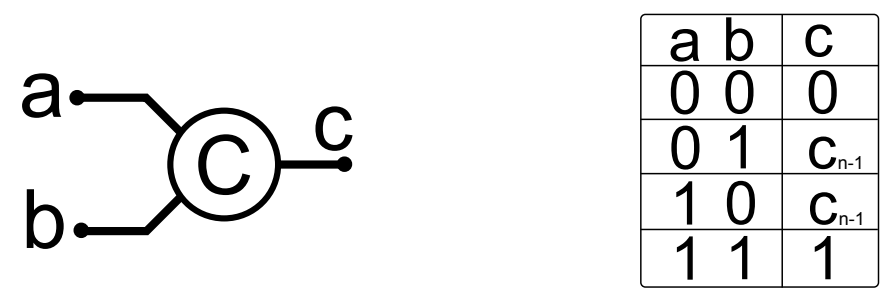

Figure 2.4: A 2-input C-element -

The Speed-Independent (SI) circuits: 


\subsection{Discussions on the delay model of SI circuits}

The DI circuits are quite limited and most practical specifications do not have DI implementations. The SI circuits are usually adopted to enlarge specifications that could be synthesized. As was proved in [24], there exists an SI implementation for any deterministic computation ${ }^{1}$. The SI circuits also allow unbounded delays on gates, but wires in a fork must have the same magnitude delay. If the delays on wires in a fork (since they have the same magnitude) are combined into the corresponding gate delay, the timing assumption behind the SI circuits is equal to say that gates in an SI could have unbounded delays but wires are instantaneous.

\subsection{Discussions on the delay model of SI circuits}

Glitches are unwanted transitions on a signal often generated by the delay variations on gates and wires. Synchronous circuits could use the clock to filter the glitches to prevent them from causing hazards. While in asynchronous circuits, especially for the circuits in input-output mode, all signals should be valid at any time and therefore any glitch could be recognized as a pre-mature transition.

The appearances of glitches are usually dependent on the delay model. The pure delay is usually considered to be a safer delay model compared to the inertial delay, because potential glitches might be absorbed by an inertial delay if they are narrow enough. But in [26], the author exemplifies that a circuit might manifest a

\footnotetext{
${ }^{1}$ Even though the Quasi Delay Insensitive (QDI) circuits [25] have different definition, specification form and synthesis flow, they behaviorally equal to the SI circuits. In this thesis, the QDI circuits are indistinguishably recognized as the SI circuits.
} 


\subsection{Discussions on the delay model of SI circuits}

hazard under inertial delay model while it would be safe under pure delay model.

One example of this situation is presented in Figure 2.5, where gate $x$ is an internal gate and gate $y$ is a primary output. There are two glitches appear on the two inputs $a$ and $b$. The output of gate $y$ is expected to stay at '1'. Assume the gate $y$ has a pure delay. If the delay model of gate $x$ is pure (case 1 in Figure 2.5), then the glitch on input $a$ will be canceled out by the glitch on gate $x$, the primary output $y$ is hazard-free. However, if the gate $x$ has an inertial delay (the gate $y$ is still under pure delay model) then the glitch on input $a$ will go through $y$ and appear at the primary output as a hazard(case 2 in Figure 2.5).

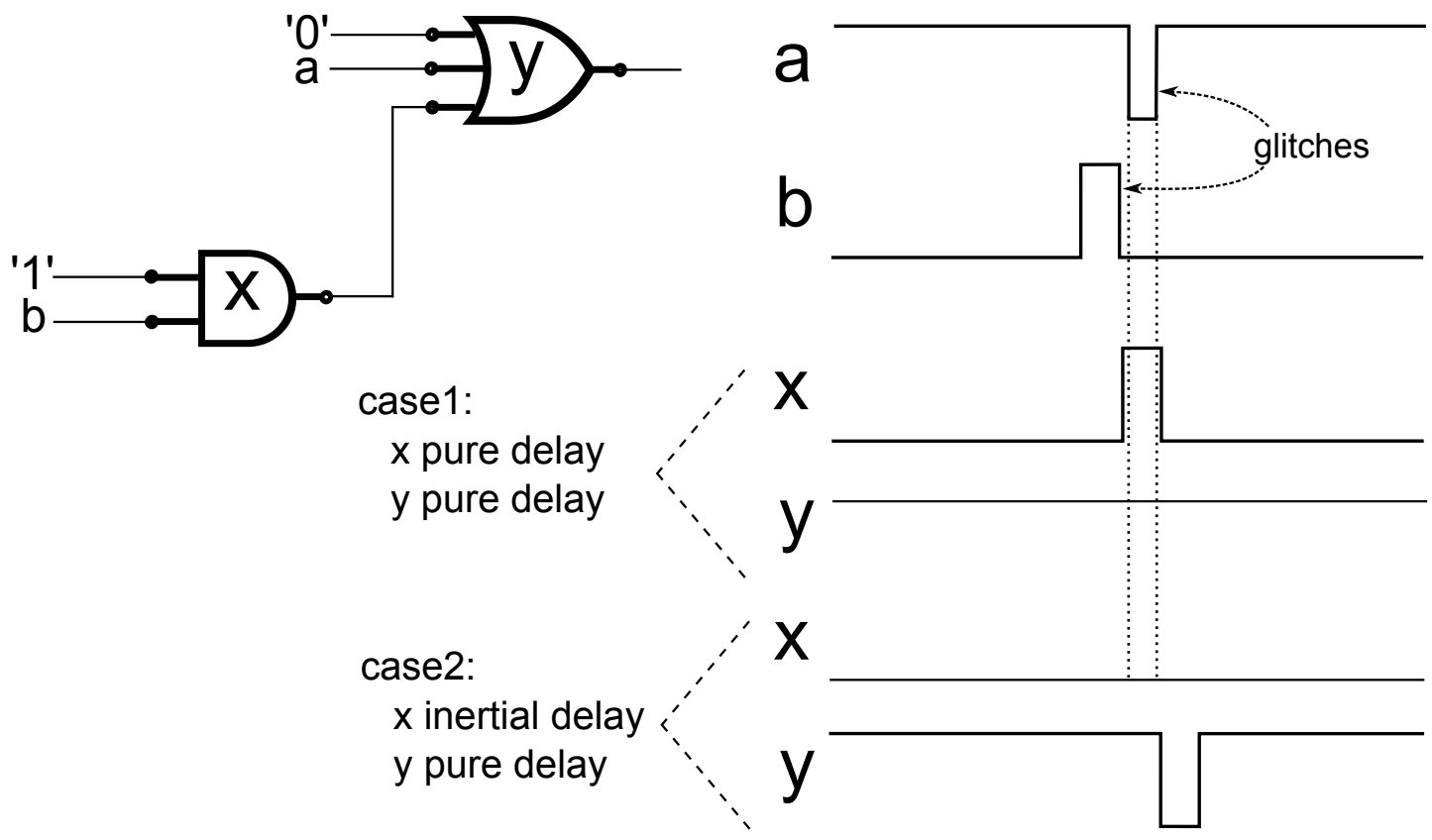

Figure 2.5: Glitches with respect to delay models -

The above example shows that the pure delay model is not always safer than the inertial delay model. But it is only true when one glitch is used to cancel out another glitch. When we discuss the glitch-free implementation (no glitches 
are allowed at any signal), the pure delay model will always be a safer mode compared with the inertial delay model. 


\section{Chapter 3}

\section{Speed-independent Circuits}

The class of asynchronous circuits is a broad class. Usually, each design paradigm has its own flow. This chapter further explains why SI design is more interesting than other asynchronous design paradigms. Also, this chapter introduces the mathematical models used in the SI design flow.

\subsection{Why SI design}

As was introduced in the previous chapter, there are many asynchronous design paradigms. Unfortunately, these paradigms have totally different design flows. Synthesis techniques for one kind of design cannot be used in others. In this thesis we adopt the SI design, which we think has the following advantages over others:

Strong variation tolerance ability:

The SI design which only has an isochronic fork timing assumption is robust 
to process variations and harsh environment.

As the technology develops, process variations become quite severe. The design paradigms that need to evaluate the real timing or compare the relative timing between similar components become unreliable. Moreover, all kinds of unreliable environments could appear as the portability of devices increases. The power supply might be from the energy harvesting devices and/or the circuit itself might operate under subthreshold voltage. The SI design has been proved to adjust well to the harsh environment, while other design paradigms are more vulnerable to variations.

As will be shown in the following chapters, the isochronic fork timing assumption could be safely relaxed into a much looser timing assumption and this timing assumption is easier to implement. The overhead to fix the potential hazards is not expensive.

Comparatively well supported by EDA tools:

One critical obstacle to asynchronous design for general use is the lake of EDA tool support. Many commercial circuits like [27] and [28] involve remarkable manual efforts. Compared with other asynchronous design paradigms, SI design is comparatively well studied and better supported by EDA tools in the design flow. For example, there are [29] [30] [31] [32] [33] and [34] for synthesis. [35] [36] [37] and [38] for decomposition and technology mapping, [39] [40] [41] and [42] for verification and [43] for testing. Almost each step in design flow is supported or partially supported by existing automation techniques. 


\subsection{Petri Net}

Petri net was first formally introduced by Carl Adam Petri in his Ph.D. thesis [44] as a modeling language for discrete distributed systems. It has strict mathematical definition and semantics as well as visually graphic representation. The explicit representations of enabling, disabling, concurrency and conflict make Petri Net quite suitable to model asynchronous systems. Especially, one particular subset of interpreted Petri Net, called the Signal Transition Graph is quite popular in describing the behavior of SI circuits. Formally,

A Petri Net $(\mathrm{PN})$ is a quadruple $N=\left(P, T, F, m_{0}\right)$, where

$P$ is a finite set of places,

$T$ is a finite set of transitions,

$F \subseteq(P \times T) \cup(T \times P)$ is a flow relation and

$m_{0}: P \rightarrow \mathbb{N}$ is the initial marking.

Places represent conditions and are usually depicted as circles $(\bigcirc)$ in graphical representation and transitions are events in a system and usually denoted by bars (-). A place $p \in P$ (transition $t \in T$ ) is an input place (transition) of a transition $t \in T$ (place $p \in P$ ) if $p \times t \in F(t \times p \in F)$ or is an output place (transition) of a transition $t$ (place $p)$ if $t \times p \in F(p \times t \in F)$. The set of input places (transitions) of a transition $t$ (place $p$ ) is denoted by ${ }^{\bullet} t(\bullet p)$ and the set of output places (transitions) of a transition $t$ (place $p$ ) is denoted by $t^{\bullet}\left(p^{\bullet}\right)$. This definition also applies to a set of places (transitions). For example, for the set of places $P_{1} \subseteq P, \bullet P_{1}=\bigcup_{p \in P_{1}} \bullet p$.

The marking of a $\mathrm{PN}$ is a function $M: P \rightarrow \mathbb{N}$, which gives each place a 
non-negative integer representing the number of tokens in this place. A place $p$ is marked if $M(p)>0$, otherwise it is blank. Graphically, a token is drawn as a $\operatorname{dot}(\bullet)$.

A transition $t$ is enabled in a marking $m$, if every place in $\bullet t$ is marked. An enabled transition may fire, which will remove one token in each place in ${ }^{\bullet} t$ and add one token to each place in $t^{\bullet}$. This firing will change the marking $m$ into a new marking $m^{\prime}$ and this transformation is denoted by $m \stackrel{t}{\rightarrow} m^{\prime}$.

A marking $m^{\prime}$ is said to be reachable from marking $m$, if there exists a firing sequence $\sigma: t_{1} t_{2} \ldots t_{n}$ which transforms the marking $m$ to $m^{\prime}$. The marking set $\mathfrak{M}$ of a PN is the set of all markings reachable from the initial marking $m_{0}$.
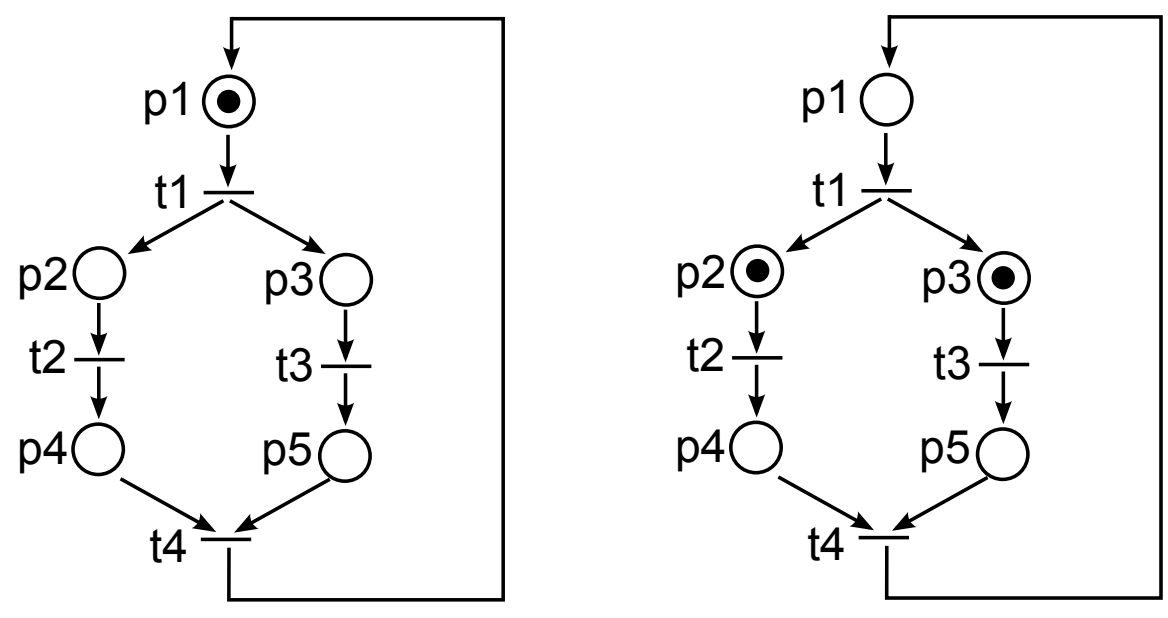

Figure 3.1: A PN example -

The PN example in the left diagram in Figure 3.1 has five places $P=$ $\left\{p_{1}, p_{2}, p_{3}, p_{4}, p_{5}\right\}$, four transitions $T=\left\{t_{1}, t_{2}, t_{3}, t_{4}\right\}$ and the initial marking $m_{0}=$ $(1,0,0,0,0) . t 1$ is the only transition that is enabled in the initial marking. When it fires, the initial marking transfers into another marking $m_{1}=(0,1,1,0,0)$ as is shown in the right diagram in Figure 3.1. The marking set of this $\mathrm{PN}$ is 
$\{(1,0,0,0,0),(0,1,1,0,0),(0,0,1,1,0),(0,1,0,0,1),(0,0,0,1,1)\}$.

Besides the basic semantics of the PN, the properties and concepts [45] introduced below are often involved when certain kinds of PN are used to depict the behavior of asynchronous circuits.

A transition $t$ is said to be live in a marking $m$, if there exists a marking $m^{\prime}$ reachable from $m$, such that $t$ is enabled in $m^{\prime}$. A PN is live if every transition is live in any reachable marking from the initial marking $m_{0}$.

A PN is safe if each place could have at most one token in any reachable marking from the initial marking $m_{0}$.

A place is said to be a choice place [46] if it has more than one output transition. A place is said to be a merge place if it has more than one input transition. A choice place is further a free-choice place if this place is the only input place of all of its output transitions. A PN is a free-choice PN if all its choice places are free-choice places. A PN is said to be a Marked Graph (MG) if it does not have any choice and merge place.

Two transitions $t_{1}$ and $t_{2}$ are in conflict, if there is a marking $m$, where $t_{1}$ and $t_{2}$ are enabled but fire one will make another from enabled to disabled in the resulting marking. $t_{1}$ and $t_{2}$ are concurrent if for all markings, where $t_{1}$ and $t_{2}$ are both enabled, they are not in conflict.

The PN shown in the left diagram of Figure 3.2 is neither live nor free-choice. The transition $t_{3}$ will never be enabled and it has two choice place $p_{2}$ and $p_{3}$ as its input places. The PN shown in the middle diagram of Figure 3.2 is not safe because every place in it could have up to two tokens; the transitions $t_{1}$ and $t_{3}$ are concurrent in this net. Finally, the PN in the right diagram of Figure 3.2 is 

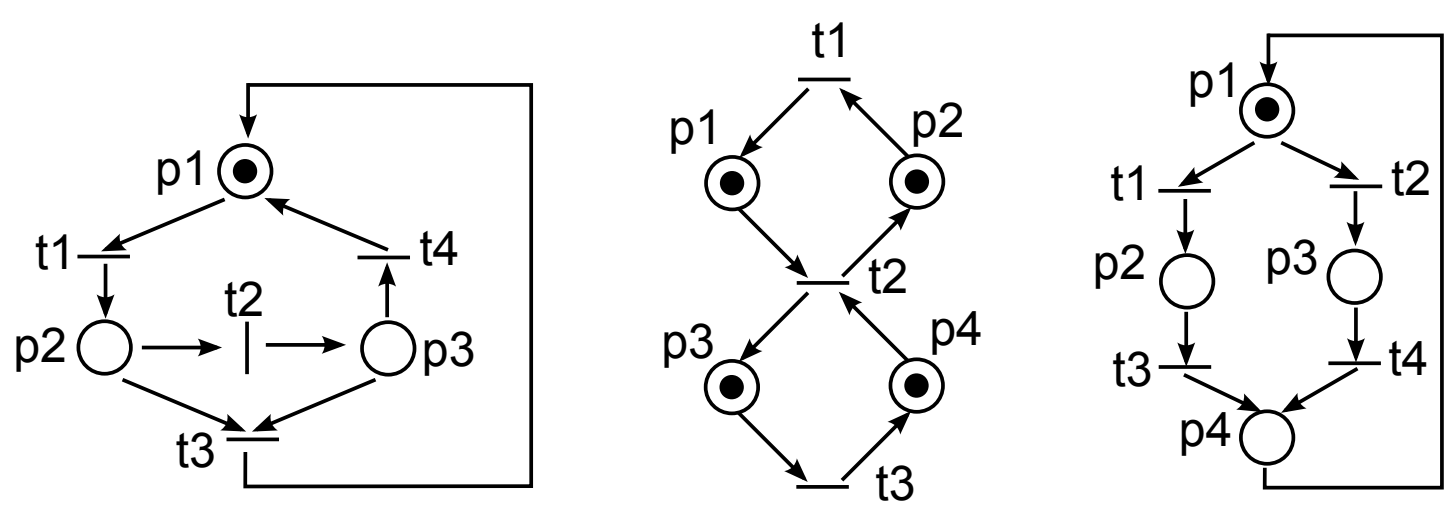

Figure 3.2: PN properties -

live, safe and free-choice and the transitions $t_{1}$ and $t_{2}$ are in conflict.

A transition $t_{1}\left(t_{2}\right)$ is a predecessor transition (successor transition) of transition $t_{2}\left(t_{1}\right)$, if $t_{1}^{\bullet} \cap^{\bullet} t_{2} \neq \varnothing$ and denoted by $t_{1} \Rightarrow t_{2}$. The set of predecessor transitions (successor transition) of transition $t$ is denoted by ${ }^{\triangleleft} t\left(t^{\triangleright}\right)$.

\subsection{Signal Transition Graph}

The signal transition graph, which is an interpreted PN, was introduced in [47] (called Signal Graph) and [48] as a high level description of asynchronous circuits. The transitions in the underlying PN are signal transitions in the circuit. Formally,

A Signal Transition Graph (STG) is a triple $G=(N, A, \lambda)$, where

$N$ is the underlying $\mathrm{PN}$,

$A$ is a finite set of signals and

$\lambda$ is a labeling function which assigns transitions in $N$ to $A \times\{+,-\}$.

For all $a \in A, a+$ depicts a rising transition (from logic low to logic high) on 


\subsection{Signal Transition Graph}

signal $a, a-$ depicts a falling transition (from logic high to logic low) on signal $a$ and $a *$ is used to depict either $a+$ or $a-$. The index $i$ (like $a * / i)$ is used to distinguish multiple occurrences of transitions on the same signal in an STG when necessary.

As a special kind of PN, STG inherits all the semantics belonging to PN. Moreover, in order to model the SI circuits, STG needs fulfilling additional requirements.

Usually, STG is produced by the designer either manually from text description, or by translating the timing diagram with some automatic tools [49]. The STG, whose signal set $A=I \cup O$, which only depicts the interactions between the circuit and the environment, is called a specification $S T G$, denoted by $S T G_{\text {spec }}$; while the STG, whose signal set $A=I \cup R \cup O$ that depicts all event orders in an SI circuit, is called an implementation $S T G$, denoted by $S T G_{i m p}$.

The PN containing non-free-choice places or unsafe places could be very complex for analyzing. In this thesis, the underlying PN of an STG is restricted to be live, safe and free-choice if not specified. One technique to process some non-free-choice STGs will be discussed in the last chapter of this thesis.

Graphically, STG is often short-handed by omitting the places which have only one input and output transitions and using labeled transitions to instead the bars in the underlying PN. Figure 3.3 shows an SI circuit (left), in which, $I=\{a, b, c\}, R=\{d\}$ and $O=\{x, y\}$. Its $S T G_{\text {spec }}$ (middle) only depicts the signal transitions on circuit's interface while its $S T G_{i m p}$ (right) depicts all the signal transitions within the circuit.

In an STG, the transitions on the same signal must have consistency. It means 
that in any firing sequences, the rising transitions and falling transitions on the same signal must appear alternatively.

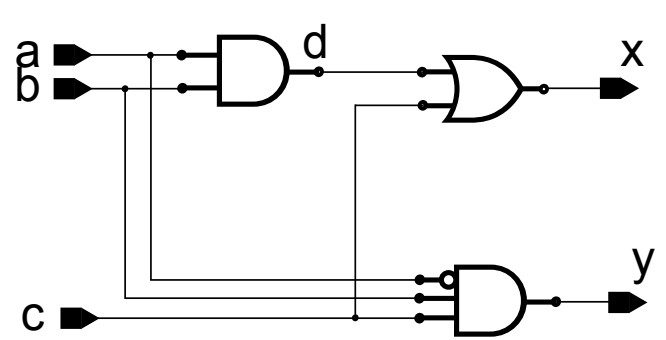

SI circuit

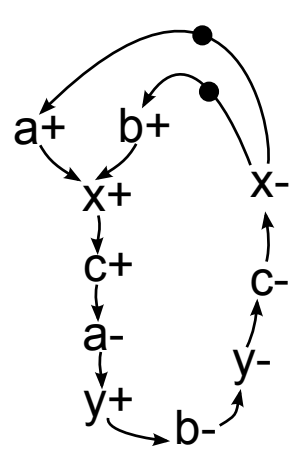

STG spec

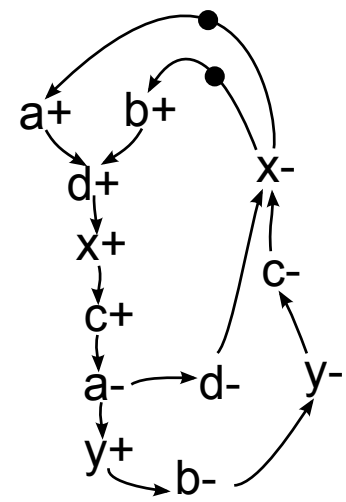

STG $_{\text {imp }}$

Figure 3.3: An SI circuit with its $S T G_{\text {spec }}$ and $S T G_{i m p}$ -

\subsection{State Graph}

An STG explicitly describes the relations between events. So, the STG is suitable for high level modeling and manipulating the behavior of an asynchronous system. While, the logic synthesis, optimization and verification are much easier to be carried on a low level model, where the reachable states of an STG are explicitly presented.

The state graph (also called the transition diagram in [47] or state transition diagram in [9]) is a binary labeled finite automaton. Each state in the finite automaton is a reachable marking of its STG. The binary value of a state represents the value of signals in the corresponding circuit.

A state graph $(\mathrm{SG})$ is a quintuple $S G=\left(A, S, E, \pi, s_{0}\right)$, where 
$A$ is a finite set of signals,

$S$ is a set of states,

$E=S \times S$ is a set of transitions,

$\pi$ is a labeling function which labels each state $s \in S$ with a bit-vector over $A$ and

$s_{0}$ is the initial state.

The value of signal $a$ in state $s$ is denoted by $s(a)$. Two states $s$ and $s^{\prime}$ are adjacent if $\left(s, s^{\prime}\right) \in E$. A transition from state $s$ to its adjacent state $s^{\prime}$ by firing $a *$ is denoted by $s \stackrel{a *}{\rightarrow} s^{\prime}$. For $s \stackrel{a *}{\rightarrow} s^{\prime}$ the triple $\left(s, a *, s^{\prime}\right)$ is said to be consistent if when $a *=a+$ then $s(a)=0, s^{\prime}(a)=1$ and $s(b)=s^{\prime}(b)$ for all $b \in A$ and $b \neq a$. An SG is considered to have a consistent state encoding if each possible triple $(s, a *, s)$ in this $\mathrm{SG}$ is consistent.

An SG of an STG could be derived by recursively firing enabled transitions from the initial marking and labeling the resulting marking set. An SG derived from an STG will have a consistent state encoding if and only if the rising and falling transitions on the same signal appear iteratively[48] in the STG.

The concept of region used data mining was introduced into the SG in [50] for classifying the states in order to accelerate the manipulations on an SG. States in each region have the same properties with respect to the corresponding signal.

Event $a *$ is excited in state $s$ if there exists a state $s^{\prime} \in S$ such that $s \stackrel{a *}{\rightarrow} s^{\prime}$. Signal $a$ is stable in state $s$ if $a *$ is not excited in $s$. A set of states $S^{\prime} \subset S$ is said to be the $i-t h$ positive excitation region of signal $a$, denoted by $E R_{i}(a+)$, if it is the $i-t h$ largest connected set of states such that for every state $s \in S^{\prime}, a+$ is excited in $s$. A set of states $S^{\prime} \subset S$ is said to be the $i-t h$ negative excitation 
region of signal $a, E R_{i}(a-)$, if it is the $i-t h$ largest connected set of states such that for every state $s \in S^{\prime}, a-$ is excited in $s$. A set of states $S^{\prime} \subset S$ is said to be the $i-t h$ positive quiescent region of signal $a+$, denoted by $Q R_{i}(a+)$, if it is the $i$ - th largest connected set of states such that for every state $s \in S^{\prime}, a$ is stable and $s(a)=1$. A set of states $S^{\prime} \subset S$ is said to be the $i-$ th negative quiescent region of signal $a-$, denoted by $Q R_{i}(a-)$, if it is the $i-t h$ largest connected set of states such that for every state $s \in S^{\prime}, a$ is stable and $s(a)=0$.

Figure 3.4 shows an STG and the SG derived from this STG. All regions defined above are explicitly shadowed.

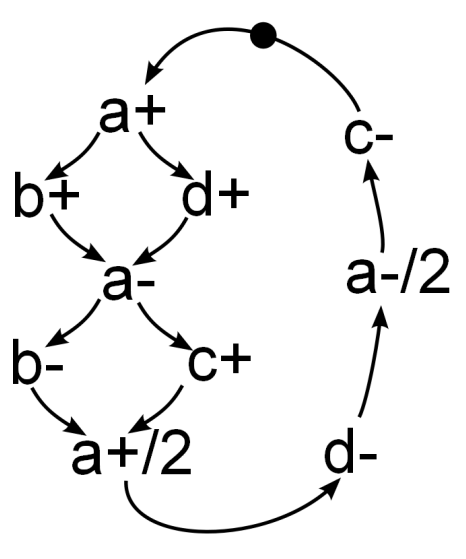

STG

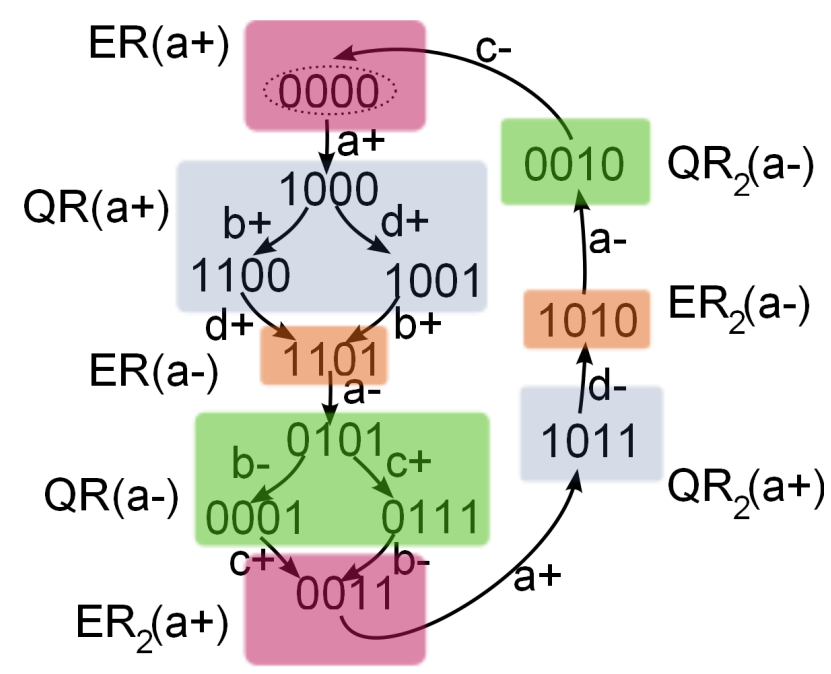

SG

Figure 3.4: An STG with its SG - 


\subsection{Summary}

This chapter introduces the popular models that are often adopted by existing methods to synthesis and verification SI circuits. The high level behavior of SI circuits is often denoted by a labeled live and safe PN called the STG. The entire reachable state of the circuit could be explicitly expressed by one kind of low level finite automaton, called the SG. In the chapter 5, the STG will be used to describe and manipulate the event causalities in an SI circuit and the hazards will be checked in the corresponding SG of the STG. The combination use of STG and SG makes the proposed method work efficiently. 


\section{Chapter 4}

\section{Timing Issues in SI Circuits}

The timing issues for SI circuits have attracted lots of attention from researchers. This chapter presents the main factors that would cause the failure of the isochronic fork timing assumption. A relaxed timing assumption that will be adopted in the following chapters will also be introduced.

\subsection{Timing assumptions in SI circuits}

The cornerstone of SI circuits is the concept of acknowledgement. We say one signal transition $a *$ is acknowledged by another signal transition $b *$ if $b *$ cannot happen until $a *$ happens. E.g. for an $A N D$ gate with inputs $a$ and $b$ and output $o$, one could say that the rising transition of $o$ will acknowledge both $a+$ and $b+$; but the falling transition of $o$ could at most acknowledge one falling transition either on $a$ - or $b$-.

The fundamental assumption of the SI circuits is so called the isochronic 
fork timing assumption, which assumes that when a transition at any branch in a fan-out fork is acknowledged, this transition will also be acknowledged at other branches in the fork. The hypothesis behind the assumption is that a transition on a gate is only required to be acknowledged by one branch in its fanout fork. Those branches which are not acknowledged explicitly are considered to be acknowledged by isochronic fork timing assumption.

\subsection{Existing research on isochronic fork reliability}

Much previous research has investigated the issues related to the isochronic fork timing assumption. On the one hand, some researchers tried to improve the circuit performance by introducing more aggressive timing assumptions to the circuits. For example, in [51], researchers extended the isochronic fork into multi level isochronic forks and in [50] concurrency reduction and lazy transition timing techniques were used to improve the circuit performance. On the other hand, some researchers investigated the reliability of the isochronic fork timing assumption [52] [53] [54] [55]. They tried to find out what aspects might cause the failure of isochronic fork timing assumption and what was the consequence when the isochronic fork timing assumption was no longer guaranteed.

The next few subsections present the possible causes that could lead to the failure of the isochronic fork timing assumption. One could conclude that: as the technology develops, isochronic forks become more and more unreliable and additional techniques must be used to guarantee the correctness of SI circuits in the near future. 


\subsubsection{Input negations}

The first observation about the violation of isochronic fork is the input negations. When a netlist is implemented, the input negations must be decomposed into individual inverters. Glitches might appear if the delays of these inverters are large enough.
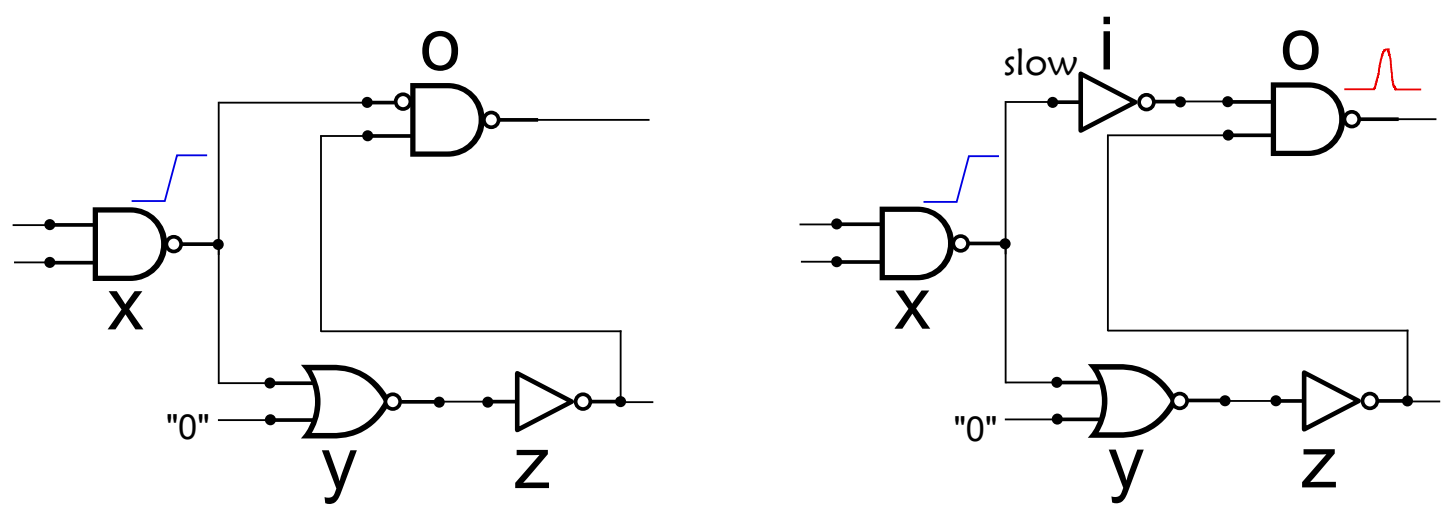

Figure 4.1: Glitches caused by inverter delay -

The left diagram in Figure 4.1 shows a circuit with initial values of the signals $x, y, z$ and $o$ at " $0 ", " 1 ", " 0 "$ and "0" respectively. The output of the gate $o$ is expected to maintain at "0" after $x+\Rightarrow y-\Rightarrow z+$. But when the input inversion bubble attached to the gate " $O "$ is decomposed into an individual gate $i$ (as is shown in the right diagram), this gate might stay at "1" while gate $z$ has risen to "1". This will cause a positive glitch at the gate $o$.

When an SI circuit is synthesized from the SG based method such as petrify [50], certain gates in the circuit are inevitable to contain input negations. In [52], the author found an error caused by the input inverter and concluded that in order to guarantee the correctness of SI circuits, certain inverters attached to 


\subsection{Existing research on isochronic fork reliability}

gate inputs must be considered having negligible delay compared to that of gates.

Much previous research has attempted to solve this problem by specification refinement [56, 57]. These works tried to introduce additional signals in the original specifications (e.g. STG) to make sure that the final synthesized circuit did not have any input bubbles requiring negligible delays. These techniques often generate compact and fast circuits. But they are not only hard to automate but also not easy to utilize manually except for experienced designers and thus cannot be considered as a general solution. Moreover, as the gate becomes faster, the interconnect wire delay could far exceed the gate delay. The consequence is when a long wire appears, its delay might be large enough for generating glitches even though no inverters appear on it.

\subsubsection{Threshold variations}

In [53], the author investigated the situations under which glitches might appear due to threshold variations. One important experiment showed that compared with the delay introduced by the input negation or wire delays, the threshold variations are more dangerous.

This situation is illustrated in Figure 4.2, where the circuit is in the same initial state as in Figure 4.1. The output of gate $o$ is expected to maintain at "0" after $x+\Rightarrow y-\Rightarrow z+$. In order to do so, the transition $x+$ must propagate to the gate $o$ before the transition $z+$ reaches the gate $o$. That is, the effect of transition $x+$ must propagate slower through the lower path than through the wire $l 1$. If one increases the length of wire $l 2$ in the fork, it will introduce extra 
delay to the lower path and should not affect the correctness of the circuit. But if the threshold voltage of gate $y$ is lower than that of gate $o$, glitches could appear when wire $l 2$ is long enough.
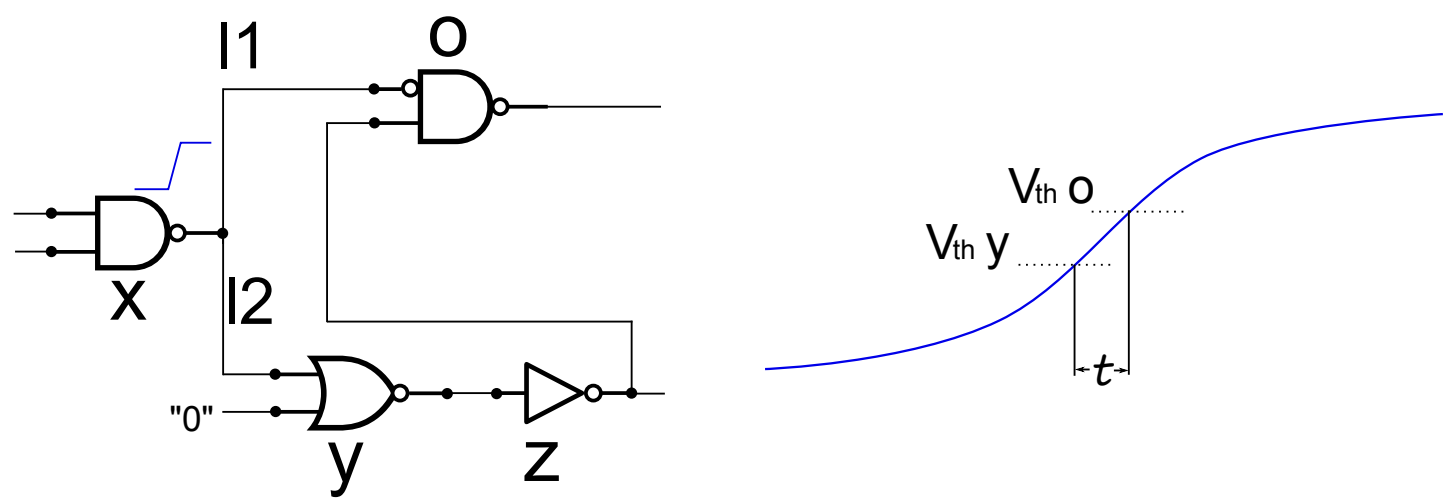

Figure 4.2: Glitch caused by threshold variation -

When the length of wire $l 2$ increases, the capacitance of the whole fork will thereby increase. A heavy capacitance will drive the transitions on gate $x$ into a slow slope. If the threshold of gate $y$ is lower than that of gate $o$, the gate $y$ would see the rising transition on $x$ before gate $o$ does. Let us denote gate $y$ sees $x+\tau$ time beyond gate $o$ as depicted in Figure 4.2. If this $\tau$ is large enough, the transition $z+$ will reach gate $o$ before $x+$. A "1" glitch will appear at gate $o$.

The threshold voltage variation is quite likely to destroy the isochronic fork assumption and make the circuit malfunction. As was reported in [22], one circuit malfunctioned due to the failure of isochronic fork requirement caused by the nonuniformity of the threshold even in $1.6 u m$ process. Now, this situation is quite severe, because the $3 \sigma$ intra-die variation of the threshold voltage could reach up to $42 \%[58]$. 


\subsubsection{Buffer insertion}

As the process shrinks, the gate becomes more and more faster, however, the wire delay does not decrease accordingly. Therefore the wire delay is quite likely to dominate the delay of a circuit. In order to improve circuit performance, buffers are inserted into the circuit to cut the long wire into small segments. The inserted buffer not only introduces extra delays on a wire (the same as the decomposed inverters) but also destroys the equipotentiality of the fork.

Figure 4.3 illustrates this situation. In Figure 4.3, wire $l 1$ is a long wire while $l 2$ is a comparatively short one. Before inserting a buffer on wire $l 1$, the response time of wire $l 1$ and wire $l 2$ is quite close. Because wire $l 1$ and $l 2$ are in the same fork, the response time of wire $l 2$ will also be slowed down by the capacitance of wire $l 1$. But when a buffer is inserted on wire $l 1$ in order to cut the long wire $l 1$ into two pieces $\left(l 1^{\prime}\right.$ and $\left.l 1^{\prime \prime}\right)$. The buffer also isolates the impedance of $l 1^{\prime \prime}$. The impedance of wire $l 1^{\prime \prime}$ will no longer influence the response time of wire $l 2$. So the wire $l 1^{\prime \prime}$ only influence the delay of the upper branch; delay difference between two branches will be enlarged. Two diagrams at the bottom of Figure 4.3 show the simulation results of the response time of wire $l 1=800 u m$ and $l 2=100 u m$ before and after inserting a buffer in the middle of $l 1$. This fork is driven by a buffer, the wire and gate feature is under $90 \mathrm{~nm}$ process. As can be seen from these two diagrams, the response time of both wires in the fork is reduced after inserting a buffer on wire $l 1$. But the differences between two branches increase significantly. 

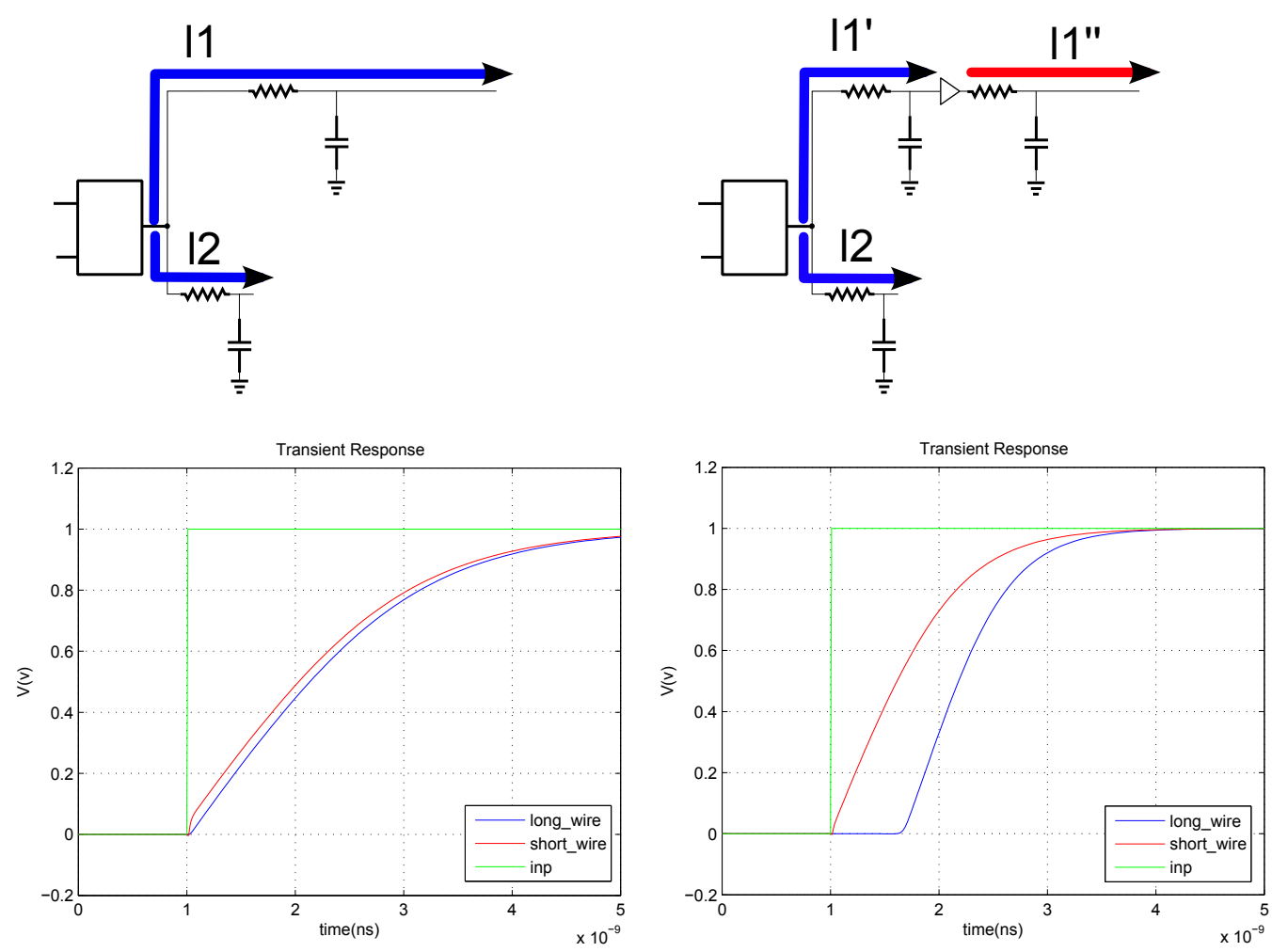

Figure 4.3: Timing difference caused by buffer insertion - 


\subsection{Recent research on isochronic fork relaxation}

As the technology develops, almost all features become more and more harmful to the isochronicity of the fan-out forks. Much recent research has investigated the situation when the isochronic fork is relaxed.

In [54], authors proposed an algorithm to find out a set of timing constraints that could guarantee the correctness of a given SI circuit when the isochronic fork timing assumption is eliminated. Their technique directly compared transitions in the STG to decide whether a circuit glitches or not. Their technique is quite restricted, as it is applicable to simple STG only, and judge whether a circuit will glitch on its high level specification will lead to inaccuracies. The hazards should be analyzed in lower level such as the SG where each reachable state is explicitly exposed. Moreover, their technique needs to represent each wire in the circuit by a signal explicitly. If this is done, the resulting STG will not be safe and thus not easy for further investigation. One example for the un-safeness is presented in Figure 4.4. An SI circuit and its STG are shown in diagrams (a) and (b). Diagram (c) explicitly denotes all wires in the circuit and diagram (d) presents the STG corresponding to (c) when the isochronic fork assumption is removed. One could see that, in diagram $(\mathrm{d})$, certain transitions $\left(a^{\prime \prime}+, c^{\prime}+, b^{\prime}-\right.$ and $\left.c^{\prime \prime}-\right)$ will not be followed by any transition. (These transitions are acknowledged by the isochronic fork timing assumption. For example, the dashed arc $a^{\prime \prime}+\Rightarrow d+$ in diagram (d) means when the transition $a^{\prime}+$ is acknowledged by $d+, a^{\prime \prime}+$ will also be acknowledged by $d+$ due to the isochronic fork timing assumption. However, this implicit acknowledgement arc cannot be treated as an ordinary arc in PN 


\subsection{Recent research on isochronic fork relaxation}

and $d+$ could fire without waiting for the firing of $\left.a^{\prime \prime}+\right)$. The input places (e.g. $\left.<a+, a^{\prime \prime}+>\right)$ to these transitions will not be safe.

In the following chapters, we adopt another timing assumption which will be explained below. This timing assumption is quite reasonable and convenient for developing an algorithm to check the hazards and generate a set of timing constraints for the correctness of a given SI circuit.

Recent research has proven that the isochronic fork timing assumption for SI circuits could be relaxed into a weaker and easier satisfiable timing assumption [55] without influencing the correctness of the circuit.

In the left diagram of Figure 4.5, wires $l 1$ and $l 2$ in a fork feed to the same gate $y$. They are called the intra-operator fork. While wires $l 1$ and $l 3$ feed to different gates. They are called the inter-operator fork [55]. In [55] the authors relaxed the isochronic fork timing assumption into the strong intra-operator fork assumption, which assumes that all the intra-operator forks in an SI circuit are isochronic.

This assumption relaxes the isochronicity hypothesis between the wires which feed to different gates. The only timing assumption is that wires in a fork that feed to the same gate are considered to be isochronic. The authors proved that any SI circuit is hazard-free under strong intra-operator fork assumption if this circuit does not have any adversary path. This is called the adversary path timing assumption.

An acknowledge path is an adversary path with respect to a wire if the path starts in the same fork with this wire, feeds to the same gate as the wire does and a transition propagates through the path faster than through the wire. 


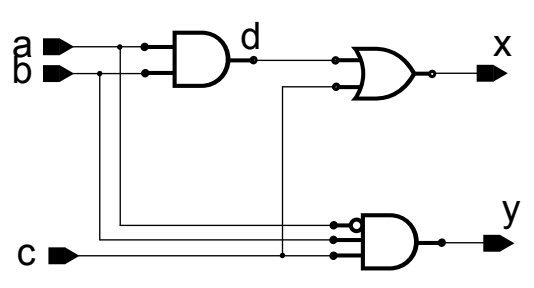

(a)

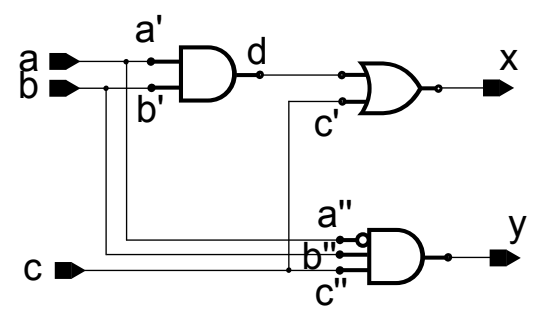

(c)

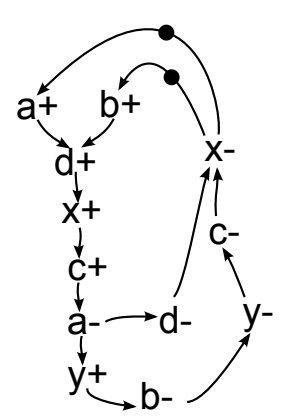

(b)

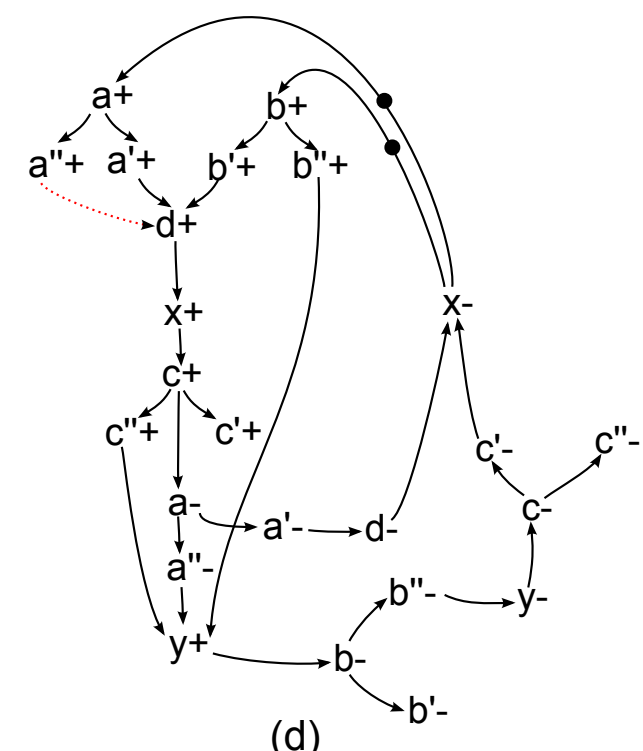

(d)

Figure 4.4: (a) An SI circuit, (b) the STG corresponding to (a), (c) The SI circuit with all wires explicitly denoted by signals and (d) the STG corresponding to (c) when the isochronic fork assumption is removed - 

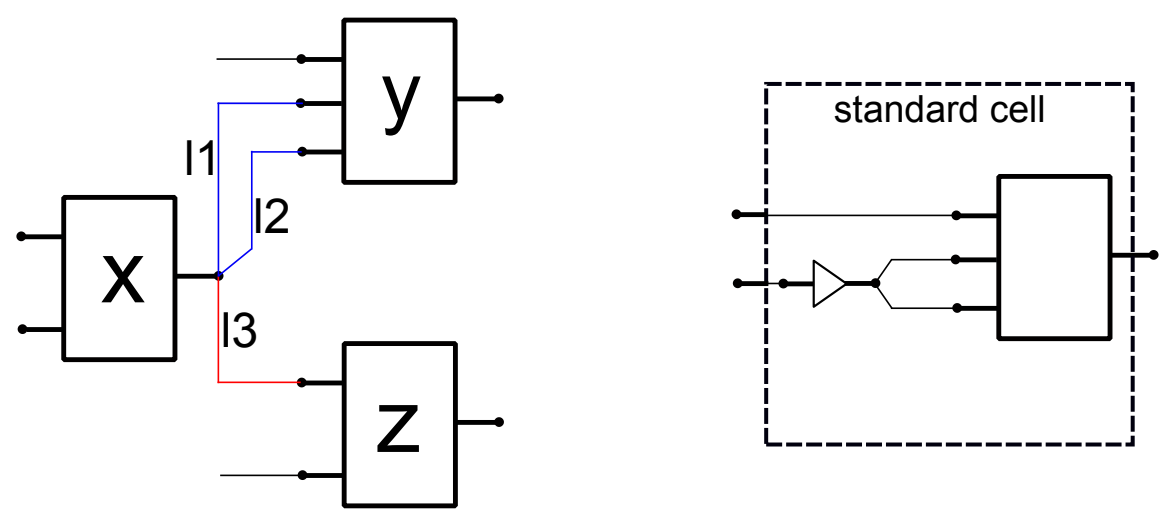

Figure 4.5: Intra operator fork timing assumption -
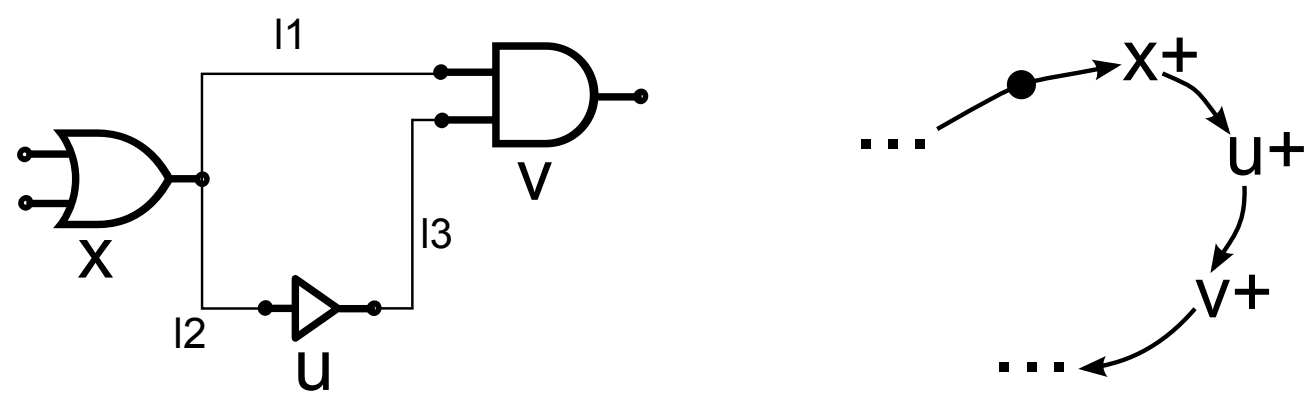

Figure 4.6: A counter-example - 


\subsection{Recent research on isochronic fork relaxation}

For example, in the left diagram of Figure 4.6, if a transition on gate $x$ propagates through wire $l 2$, gate $u$, and wire $l 3$ faster than through the wire $l 1$, then the path wire $l 2$, gate $u$ and wire $l 3$ is an (3-level) adversary path with respect to wire $l 1$.

In [55], authors proved the equivalence of the isochronic fork timing assumption and the adversary path timing assumption. They also claimed that the adversary path timing assumption is the weakest timing assumption that is both necessary and sufficient for the correctness of SI circuits.

Their work provides a good direction on hazard detection when the isochronic fork is relaxed but the problem still exists:

Firstly, the adversary path timing assumption is neither the weakest nor a necessary timing assumption for SI circuits. The isochronic fork timing assumption is only a sufficient but not a necessary timing assumption for the correctness of SI circuits. So, the equivalence with isochronic fork timing assumption cannot prove the necessity of the adversary path timing assumption with respect to SI circuits. In Figure 4.6, the left diagram shows a circuit and the right diagram shows its STG segment. If the transition $u+$ caused by $x+$ reaches the gate $v$ before $x+$ arrives at gate $v$, then the wire $l 2$, gate $u$ and wire $l 3$ is an adversary path with respect to the wire $l 1$. But this adversary path will not cause any hazard in the circuit. This counter example shows that the adversary path timing assumption includes some unnecessary timing constraints and could still be relaxed.

Secondly, in [55] authors only suggested a condition but did not propose any method to find out all potential adversary paths in a given SI circuit under a 
specification.

In the following chapters, we will relax the isochronic fork timing assumption into the intra-operator fork timing assumption as [55] did, and propose a method to obtain the weakest timing constraints that could guarantee the correctness of an SI circuit. The reason we adopt the intra-operator fork timing assumption is that the delay variations (caused by wire length variations, threshold variation and so on) are more controllable to the wires that feed into the same gate than those feed to the different gates. The wires in a fork that feed to the same gate are usually in the same length range and the threshold variation of transistors in a single gate is not that bad. If the threshold variation of transistors in a single gate is severe in the future, the standard cell of a gate could be made to contain a buffer to limit the capacitance of the fork as shown in the right diagram in Figure 4.5.

\subsection{Summary}

This chapter mainly introduces the aspects that could cause the failure of the isochronic fork timing assumption and reviews the existing research about the timing issues related to SI circuits. All these aspects will become worse as the semiconductor technology develops. So, the isochronic fork timing assumption must be relaxed into a reasonable weaker timing assumption. The intra-operator fork timing assumption proposed by existing research will be adopted in this thesis. The correct implementation of asynchronous circuits under severe process variations could be achieved by designing them in SI mode using the existing 
methods and tools first, and then relaxing the isochronic fork timing assumption and generating a set of sufficient timing constraints for the circuit to work correctly under the new timing assumption. The technique to generate the set of timing constraints will be introduced in detail in the next chapter. 


\section{Chapter 5}

\section{Hazard checking method}

A small flaw in a circuit could cause heavy cost. A famous example is the Pentium FDIV bug, which cost over $\$ 500$ million. Verification is the procedure which checks whether a circuit behaves according to its specification. As the complexity of the integrated circuit grows, verification is much more important. This is especially important for asynchronous designs, which often involve error-prone manual efforts.

However, verification is often computationally expensive, especially for asynchronous circuits. Without latches to cut the entire circuit into small pieces, the verification usually needs to consider the circuit as a whole. This makes exploration of the possible reachable states exponential to the total number of signals for highly concurrent circuits. Verification is costly even for moderate size circuits. 


\subsection{Introduction}

Verification is an important step in circuit design, which checks whether a circuit conforms to its specification. The verification of SI circuits is often carried in two steps [40] [39]. The first step checks whether the logic of the synthesized circuit satisfies the specification (functional correctness). The second step checks whether the circuit is SI, namely hazard-free under isochronic fork timing assumption (behavioral correctness). Existing techniques often use different approaches such as hierarchical verification [39] or unfolding [38] to avoid exploring the entire reachability space. However, the isochronic fork assumption is challenged by the shrinking technology. The timing verification for SI circuits should also consider the situation when the isochoric fork assumption is no longer guaranteed. Indeed, all SI circuits that are not DI will exhibit hazards when the isochronic fork assumption is violated. The task of this research is that given an SI circuit and its specification, to find a set of timing constraints such that when the isochronic fork timing assumption is violated, the circuit is still hazard-free. These timing constraints should be as loose as possible.

There are some recent publications which investigate the timing issues when the isochronic fork assumption is relaxed. For example, [59] investigates the cost by changing the interfaces between logic blocks from SI to DI, in which some conditions related to relaxing SI into DI on STG are discussed. [54] attempts to investigate the technique that could generate timing constraints that would be sufficient for the correctness of SI circuits when the isochronic fork is relaxed. The technique proposed in [54] tries to find all unacknowledged transitions appearing 
at one gate's fan-ins and judges whether these unacknowledged transitions would cause hazards. However, this technique judges the hazards directly at the higher specification level (STG level) where some important properties such as ORcausality are invisible. [55] gives a formal proof that the isochronic fork timing assumption could be replaced by a weaker "adversary path timing assumption" without affecting the correctness of the circuit. However, without considering the function of the gates in the circuit, the adversary path timing assumption is still too strong.

\subsubsection{Overall flow}

The overall flow of the proposed method for designing SI circuits under severe variations in deep-submicron technology process is shown in Figure 5.1.

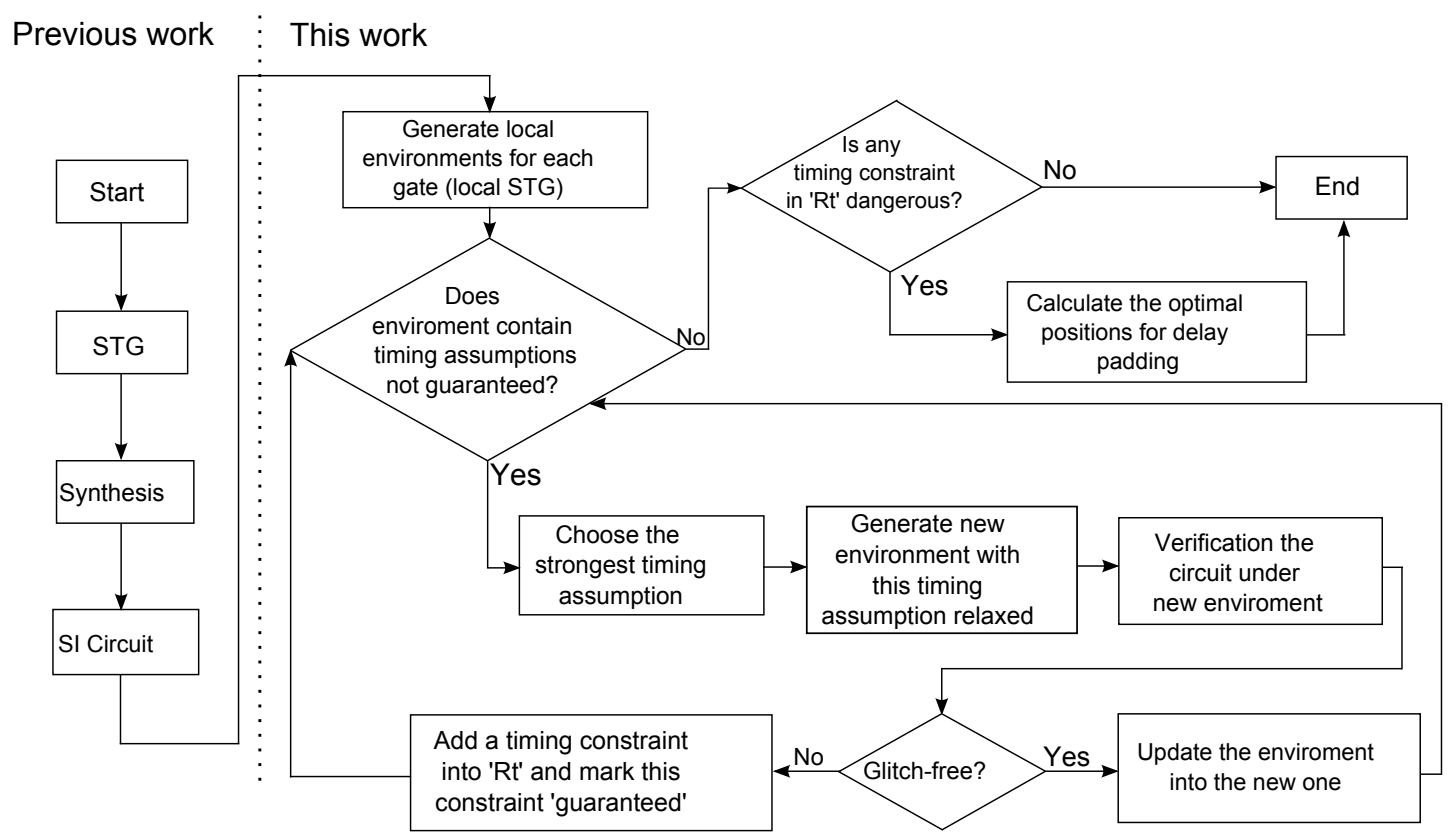

Figure 5.1: The entire flow of this work - 
The starting point of this work is a high level STG, which specifies the desired behavior of the circuit, and a corresponding SI circuit (The circuit is required to be behaviorally correct with respect to the STG specification. This could be done by existing verification tools such as [40] [39] and will not be checked in this work).

Currently, the STG that describes an SI circuit needs to be free-choice as the technique presented in this work requires to decompose the STG into a set of MGs that cover this STG. No algorithm to decompose a non-free-choice PN into a set of MGs has been published. One technique to process some non-free-choice STGs will be discussed in the Chapter 8 .

As the events in the STG consist of transitions on gate variables, each transition on gate variable must be followed by another one. This indicates that there will be a local STG for each gate, whose transitions are only these transitions on its fan-ins and fan-out signals. A local STG explicitly depicts the ordering relations of the transitions on this gate and could be derived by projecting each MG component of STG on the variables related to this gate. The local STG could be seen as the local environment of a gate, which provides input transitions to the gate and receives output transitions from the gate.

Then the local STG is checked to see whether it contains timing orderings that rely on isochronic fork assumption. If it does, the tightest timing ordering (most likely to be violated due to the process variations) will be picked and a new STG with this timing ordering relaxed will be generated.

The circuit will then be checked under the newly generated STG to see whether it is hazard-free. If true, the new STG will be accepted which has 
one adversary path less than the old STG. If not true, this timing ordering must be guaranteed for the correctness of the circuit. A timing constraint which depicts this ordering will be added into the Relative timing constraint $(R t)$ set and this timing orderings will be marked as "guaranteed already". STG splitting is required when the OR-causality appears at the relaxed STG. This situation will be discussed in detail in the following sections.

The process terminates when no timing orderings in the final STG rely on isochronic fork assumption (guaranteed by acknowledgement or by timing constraints). The possible use of delay padding is the final step, which will be briefly discussed in section 5.7.

\subsection{Deriving the Local STG}

In this thesis, the STG is used as the high level specification of an SI circuit. An STG that only describes how a circuit $C$ behaves with its environment through interface protocol is called the specification STG denoted by $S T G_{\text {spec }}$. The specification STG depicts the transition relations on primary input and output signals and is often used as a high level function description of the desired circuit. An SI circuit could be synthesized from a specification STG manually by an experienced designer or automatically by EDA tools such as petrify [60]. The synthesized circuit will have one signal for each internal gate besides the input and output signals. An STG that depicts the transition relations of all signals of a circuit $C$ (input, output and internal signals) is called the implementation STG of the circuit and is denoted by $S T G_{i m p}$. 


\subsubsection{Decomposition of a free-choice STG into MGs}

The choice places in a free-choice PN present the uncertainty of the orderings between events in this PN. One and only one successor transition of a (safe) marked choice place will eventually fire and it is uncertain which successor transition will fire in any one run. In this research, events in an STG are required to have certain relations, the ambiguities brought by the choice places in a free-choice PN need to be removed first.

An STG is a high level abstraction of all possible firing traces of a circuit under a certain environment. Due to the properties of SI circuits, choice places could only appear in the preset of input signal transitions in a free-choice STG. So, a free-choice STG could be recognized as the composition of multiple subSTGs where each subSTG represents one firing option at a choice place. Each choice place can be decomposed to derive a set of subSTGs where that choice place does not exist. The subSTGs are called the MG components of the STG, where the ordering relation between any two transitions is certain and explicit. Hack introduced an algorithm in [8], which decomposes a free-choice PN into MG components by MG allocation.

A PN $N^{\prime}=\left(P^{\prime}, T^{\prime}, F^{\prime}, m_{0}^{\prime}\right)$ is a subnet of $\mathrm{PN} N=\left(P, T, F, m_{0}\right)$ if $T^{\prime} \subseteq T$, $P^{\prime} \subseteq P$ and $F^{\prime}=F \cap\left(\left(P^{\prime} \times T^{\prime}\right) \cup\left(T^{\prime} \times P^{\prime}\right)\right)$.

A subnet $N^{\prime}=\left(P^{\prime}, T^{\prime}, F^{\prime}, m_{0}^{\prime}\right)$ of $N=\left(P, T, F, m_{0}\right)$ is a transition generated subnet of $N$ if for each $t^{\prime} \in T^{\prime}$ we have $t^{\prime}={ }^{\bullet} t$ and $t^{\bullet \bullet}=t^{\bullet}$. A transition generated subnet $N^{\prime}$ is an $M G$ component of $N$ if $N^{\prime}$ is an MG.

A set of MG components cover a PN $N$ if every transition in $N$ is in at least 
one component.

An MG allocation on a free-choice net $N=\left(P, T, F, m_{0}\right)$ is a function allo: $P \mapsto T$ such that allo $(p) \in p^{\bullet}$. For every place $p$ only one of its output transitions is allocated, others are eliminated in the allocation. Let eli $(T)$ and $\operatorname{eli}(P)$ denote the set of eliminated transitions and eliminated places, the MG reduction algorithm in [8] works like this

First step, eliminate all unallocated transitions: $\forall p \in P: p^{\bullet} \backslash \operatorname{allo}(p) \subseteq \operatorname{eli}(T)$,

Second step, eliminate the places whose input transitions are all eliminated: - $p \subseteq \operatorname{eli}(T) \Leftrightarrow p \in \operatorname{eli}(P)$

Third step, eliminate the transitions which has at least one input place eliminated: $\bullet t \cap \operatorname{eli}(P) \neq \varnothing \Leftrightarrow t \in \operatorname{eli}(T)$.

This reduction procedure starts from the first step and repeats second and third steps until eli(T) and eli $(P)$ do not change any more.

One free-choice PN and its MG components are shown in Figure 5.2.

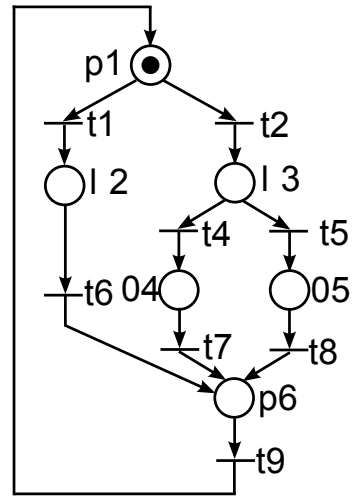

(a)

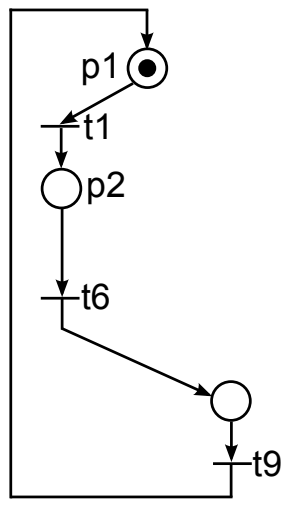

(b)

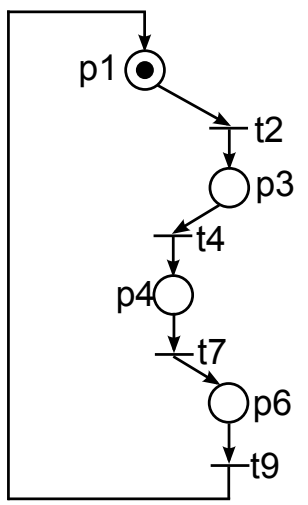

(c)

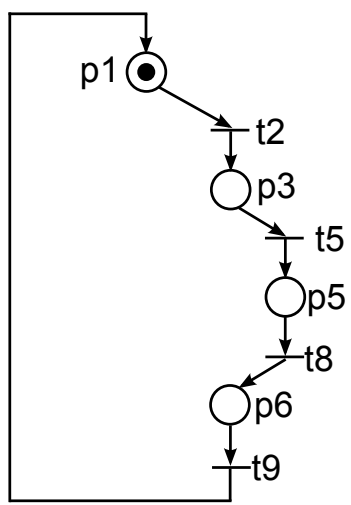

(d)

Figure 5.2: A live and safe free-choice PN (a), and its MG components (b)-(d) - 


\subsubsection{Projecting MG components on operator signals}

The implementation STG explicitly shows the transition relations of all signals in a circuit. However, the value of a gate signal only depends on its fan-in and fan-out signals. In an SI circuits, each gate could be seen interacting only with its local environment, which provides input transitions to the gate's fan-ins and expects output transitions from the gate's fan-out. This local environment only contains the signal transitions related to this gate and hides all other signal transitions in the circuit. It could be deduced that if every gate behaves according to the specification (not generating the premature transitions on its fan-out,thus providing a "correct" environment for the next level gates), the entire circuit will satisfy the specification. This local environment of a gate hides all irrelevant transitions and thus could be directly used to analyze the ordering causalities of transitions on the gate. The local environment of a gate $o$ is called the local STG of $o$ and could be derived by hiding all signals except for $X=o \cup$ fan-in (o) by projecting the $S T G_{i m p}$ on signals $X$.

Here, we review some notations which are introduced in the previous chapters. A transition in an STG is denoted by its label, like $t+$, where $t$ is the underlying signal of the transition and + or - is used to denote the direction of the transition. The notation $t *$ is used to denote either a rising transition $t+$ or a falling transition $t$ - on signal $t$. The predecessor transitions (successor transitions) of a transition $t *$ denoted by ${ }^{\triangleleft} t *\left(t *^{\triangleright}\right)$ is a set of transitions: $t^{\prime} * \in^{\triangleleft} t * \Leftrightarrow t^{\prime} *^{\bullet} \cap \bullet * \neq \varnothing($ $\left.t^{\prime} * \in t *^{\triangleright} \Leftrightarrow t *^{\bullet} \cap \bullet^{\prime} * \neq \varnothing\right)$.

In an MG component, all places are omitted and an $\operatorname{arc} t_{1} * \Rightarrow t_{2} *$ is used to 
denote the relation $t_{1} * \rightarrow p \rightarrow t_{2} *$ in the underlying PN. The arc $t_{1} * \Rightarrow t_{2} *$ has a token if the place $p$ has a token.

The projection of a marked graph $M G$ on a subset of signals $X$ is depicted in Algorithm 1. The algorithm picks each transition in $M G$ (line 1) and eliminates this transition if the signal of this transition is not in $X$ (line $2-19$ ). The function insert arc $\left(t_{1} * \Rightarrow t_{2} *, M G\right)$ in line 5 inserts a new place $p_{\text {new }}$ and two relations $t_{1} * \rightarrow p_{\text {new }}$ and $p_{\text {new }} \rightarrow t_{2} *$ into the underlying PN of $M G$. The function delete arc $\left(t_{1} * \Rightarrow t_{2} *, \mathrm{MG}\right)$ in line 13 and 16 removes the place $<t_{1} *, t_{2} *>$ and two relations $t_{1} * \rightarrow<t_{1} *, t_{2} *>$ and $<t_{1} *, t_{2} *>\rightarrow t_{2} *$ from the underlying PN of $M G$. The function eliminate_redundant_arc $(M G)$ eliminates the redundant places in $M G$, which will be introduced in detail in section 5.3.3.

Figure 5.3 shows the process of projection of an STG segment on $X$ where signal $t \notin X$.

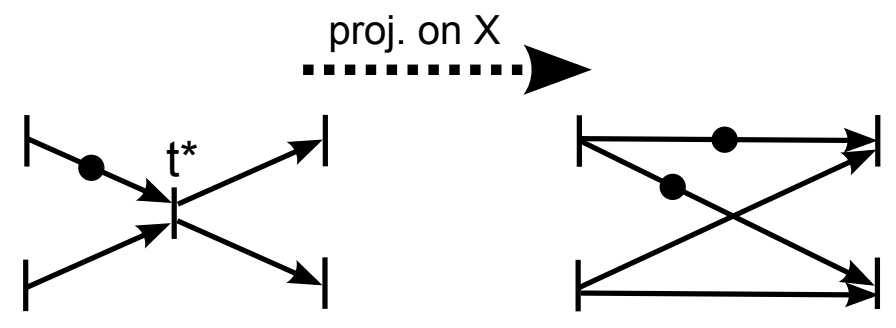

Figure 5.3: Projection of an STG segment on $X$ and $t \notin X$ -

\subsection{Timing ordering relaxation}

In the local STG (MG component) of a gate, the relations between the input and output events are explicitly depicted. There could be four kinds of arcs in a local 


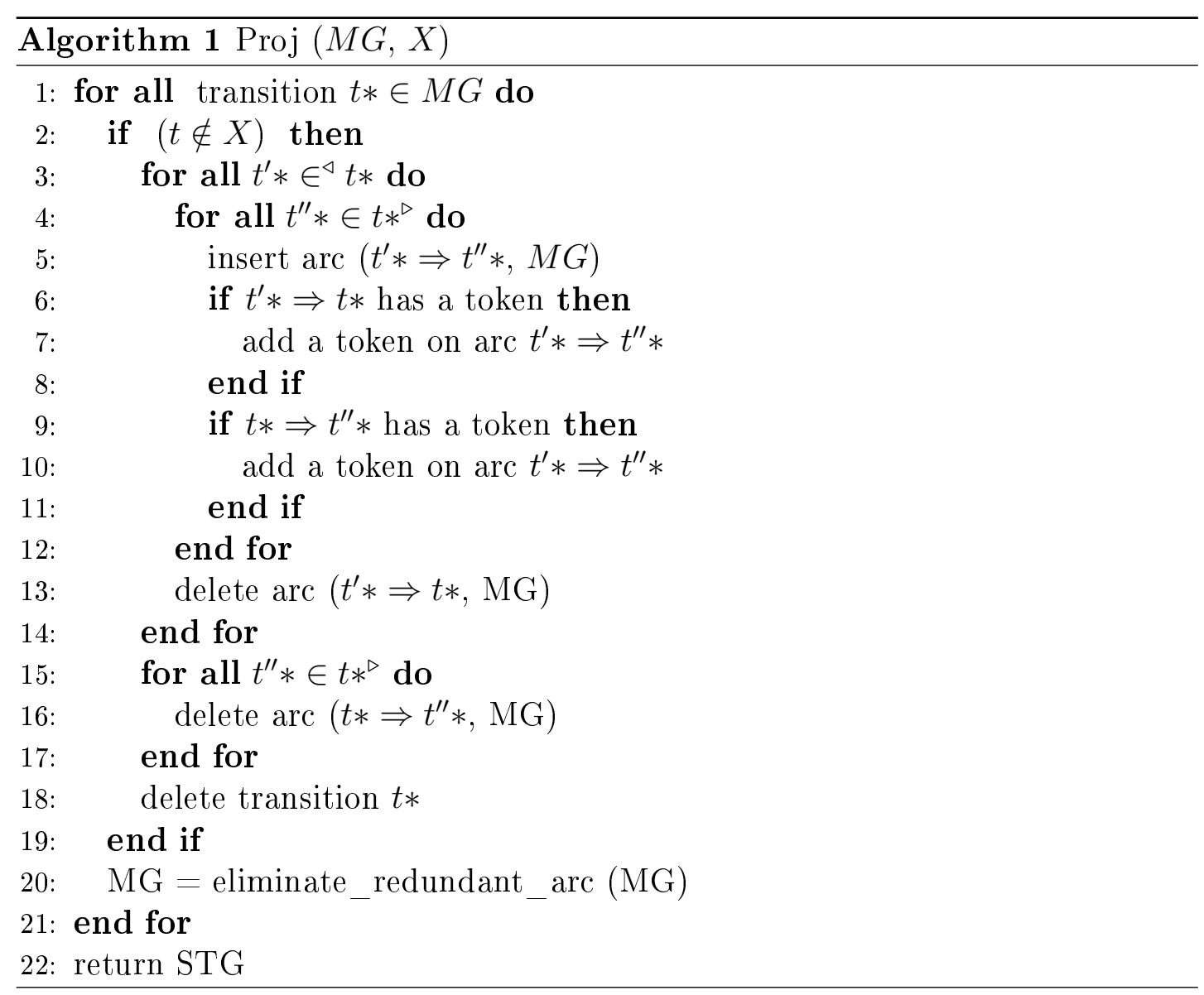


STG, which will be analyzed in detail in the following subsection.

\subsubsection{Classification of arcs in the local STG}

In the local STG of gate $a$, events are the transitions on input and output signals. There could be four kinds of arcs in the STG.

(1) $x * \Rightarrow a *$, where $x \in$ fan-in $(a)$. This kind of arc denotes an acknowledgement relation. The output transition $a *$ will only occur, when the gate receives the input transition $x *$. The timing order denoted by this kind of arc ( $x *$ fires before $a *$ ) will always be fulfilled.

(2) $a * \Rightarrow y *$, where $y \in$ fan-in $(a)$. This kind of arc denotes the interactions between the gate and its environment. The transition $y *$ will only be generated by its environment after the environment sees the transition $a *$ on the gate. Also the timing order denoted by this kind of arc will always be fulfilled.

(3) $x * \Rightarrow y *$, where $x, y \in \operatorname{fan}-\mathrm{in}(a)$ and $x=y$. This kind of arc denotes a timing order on the same input signal of gate $a$. No matter how large the delay is on a wire, the transitions on the same wire will never be reversed by this delay. So, the timing order denoted by this kind of arc will always be fulfilled.

(4) $x * \Rightarrow y *$, where $x, y \in \operatorname{fan}-\mathrm{in}(a)$ and $x \neq y$. This kind of arc denotes a timing order that the transition $x *$ will reach gate $a$ before $y *$. This indicates that there is a path, starting from gate $x$ ending at gate $y$, that $y *$ needs the occurrence $x *$ to pass along this path. The timing order behind this kind of arc might be reversed if the delay of the path is greater than the delay of the wire between gate $x$ and gate $a$, if so, the path is considered to be an adversary path 
in $[55]$.
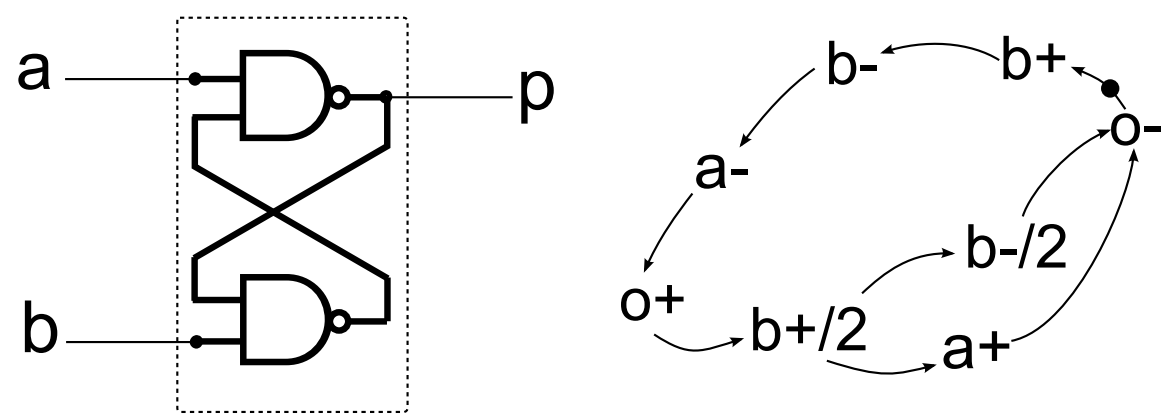

Figure 5.4: An $\overline{S R}$-latch with its local STG -

The Figure 5.4 shows an $\overline{S R}$-latch ${ }^{1}$ together with its local STG. In this example, $\{a-\Rightarrow o+, a+\Rightarrow o-, b-/ 2 \Rightarrow o-\}$ are type (1) arcs, $\{o-\Rightarrow b+$, $o+\Rightarrow b+/ 2\}$ are type (2) arcs, $\{b+\Rightarrow b-, b+/ 2 \Rightarrow b-/ 2\}$ are type (3) arcs and $\{b-\Rightarrow a-, b+/ 2 \Rightarrow a+\}$ arc type (4) arcs.

\subsubsection{Arc relaxation algorithm}

Each type (4) arc in the local STG represents a timing order between two events that relies on isochronic fork timing assumption. For example, in Figure 5.5, the $\operatorname{arc} x * \Rightarrow y *$ indicates that $y *$ is caused by the event $x *$ along an acknowledgement path (shown as the dashed line in Figure 5.5), and because of the isochronic fork timing assumption, $x *$ is guaranteed to reach the gate $o$ before $y *$. When the

\footnotetext{
${ }^{1}$ The $\overline{S R}$-latch is considered to be an atomic gate in the library as denoted by the dashed line and is internally hazard-free. Also, the '00' input combination is not restricted, because according to the definition (any gate has only one output) only one output will be used, the outputs of two NAND gates do not need to be complemented. The potential races introduced by the input combination from '00' to '11' will be excluded by disallowing this hazardous concurrency between $a+$ and $b+$ according to the criterion introduced in section 5.4.
} 

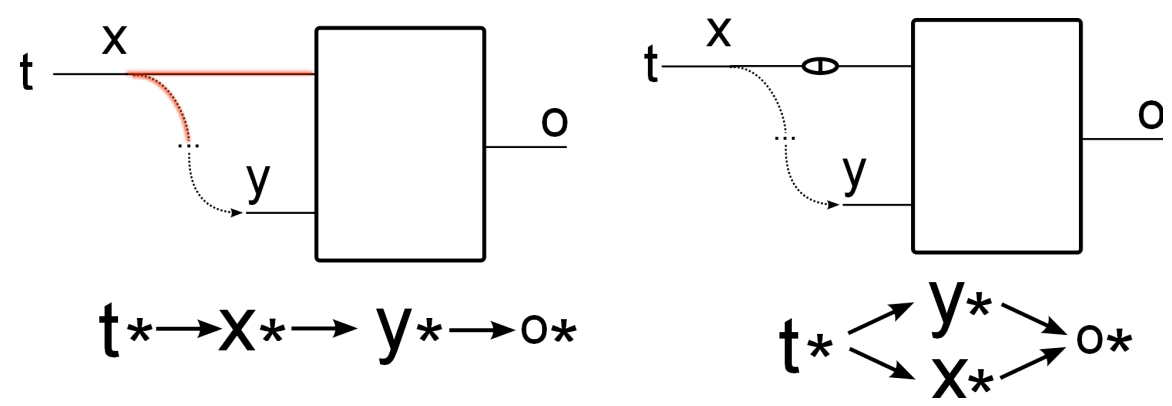

Figure 5.5: Demonstration of a type (4) arc-

isochronic fork timing assumption is violated (modeled by a delay shown in the right diagram), $x *$ and $y *$ could reach the gate $o$ in any order. In an STG, this equals to making the ordered events $x *$ and $y *$ concurrent and keeping any other relations unchanged. The modified STG would be a new specification for the gate, which allows the event $y *$ to reach the gate before $x *$.

Relaxation of an $\operatorname{arc} x * \Rightarrow y *$ such that $x, y$ are all input signals and $x \neq y$ in an marked graph $M G$ :

1) Delete the arc $x * \Rightarrow y *$ in $M G$.

2) For all transitions $b_{i} *$ such that there exists an $\operatorname{arc} b_{i} * \Rightarrow x *$ in the underlying $\mathrm{PN}$ of MG, add the arc $b_{i} * \Rightarrow y *$ into the $\mathrm{PN}$ and mark the place $<b_{i} *, y *>$ if place $\left\langle b_{i} *, x *>\right.$ or $\langle x *, y *>$ is marked.

3) For all transitions $d_{i} *$ such that there exists an $\operatorname{arc} y * \Rightarrow d_{i} *$ in the underlying PN of MG, add the arc $x * \Rightarrow d_{i} *$ into the PN and mark the place $<x *, d_{i} *>$ if place $<y *, d_{i} *>$ or $\langle x *, y *>$ is marked.

In general, the relaxation of an arc $x * \Rightarrow y *$ in an STG is shown in Figure 5.6 and detailed in Algorithm 2.

This relaxation operation of an $\operatorname{arc} x * \Rightarrow y *$ makes two ordered transitions $x *$ 

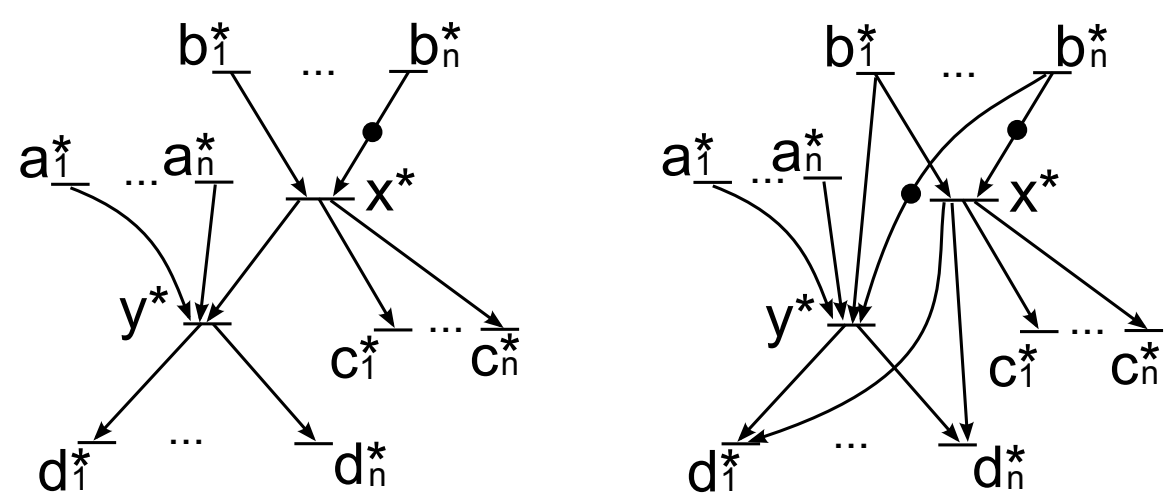

Figure 5.6: relaxation of arc $x * \Rightarrow y *$ in the most general case -

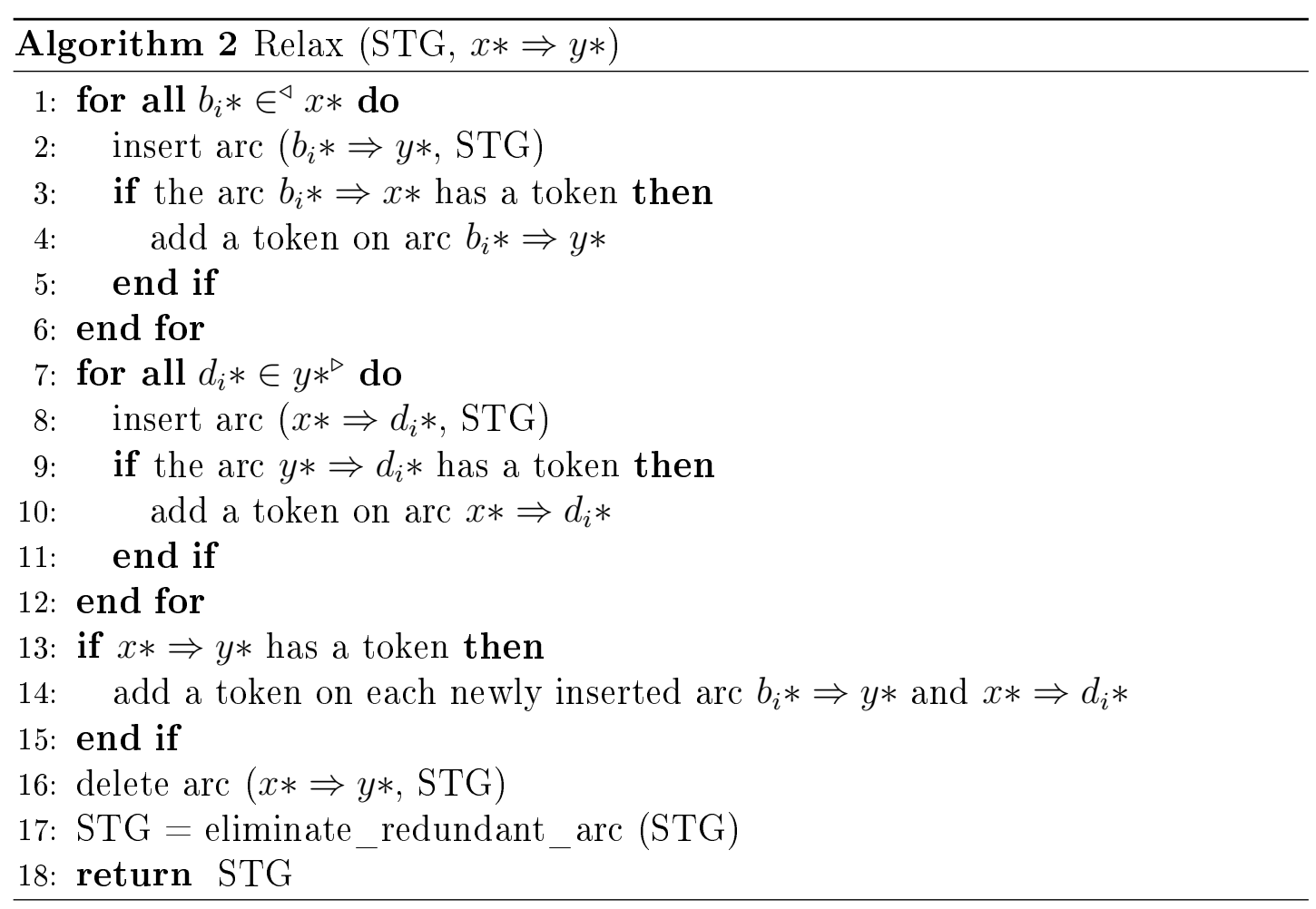




\subsection{Timing ordering relaxation}

and $y *$ concurrent and does not change their order relations with other transitions.

Lemma 1: The relaxation of an $\operatorname{arc} x * \Rightarrow y *$ in a live and safe MG component of a local STG does not influence the consistency and liveness.

Proof:

Consistency: According to the relaxation operation, the arc between the same signal (e.g. $a+\Rightarrow a-$ ) will not be relaxed. In any trace, if the rising and falling transitions of a signal alternate in the original MG component, then they alternate after relaxation. So, relaxation does not influence the consistency.

Liveness: The relaxation will delete one place $\langle x *, y *\rangle$ from the MG and add a set of places $\left\{<b *, y *>\mid \forall b * \in^{\triangleleft} x *\right\}$ and $\left\{<x *, d *>\mid \forall d * \in y *^{\triangleright}\right\}$ into the MG as was illustrated in Figure 5.6 and this relaxation does not change the liveness of the $\mathrm{MG}$,

If one transition $t \in T$ is dead in the initial marking $m_{0}$, then $\exists p \in \bullet: \forall m \in$ $\mathfrak{M}, m(p)=0$, where $\mathfrak{M}$ is the marking set of the initial marking $m_{0}$. The only places added into the MG are the places in subset of $\bullet y$ and ${ }^{\bullet}\left(y *^{\triangleright}\right)$, so, the only transitions that might become dead after relaxation are $y *$ and $y *^{\triangleright}$.

Without loss of generality, let us consider the newly added place $p_{a}=<$ $b_{1} *, y *>$ in 5.6. If $\forall m \in \mathfrak{M} m\left(p_{a}\right)=0$, due to the relaxation algorithm, the following case must be true, $m_{0}(<x *, y *>)=0 \wedge m_{0}\left(<b_{1} *, y *>\right)=0 \wedge b_{1} *$ is dead. Thus if the MG after relaxation is not live then the original MG must also contain dead transitions.

However, the relaxation of an arc might destroy the safeness of the original MG, more than one token could be accumulated at a place.

One example is shown in Figure 5.7, where the newly added arc $q-\Rightarrow a+$ 
is not safe when the arc $q-\Rightarrow p+$ is relaxed in the original graph. However, the relaxed MG will only become unsafe when the gate has redundant literals, where redundant means that these literals could be removed without affecting the behavior of the gate.

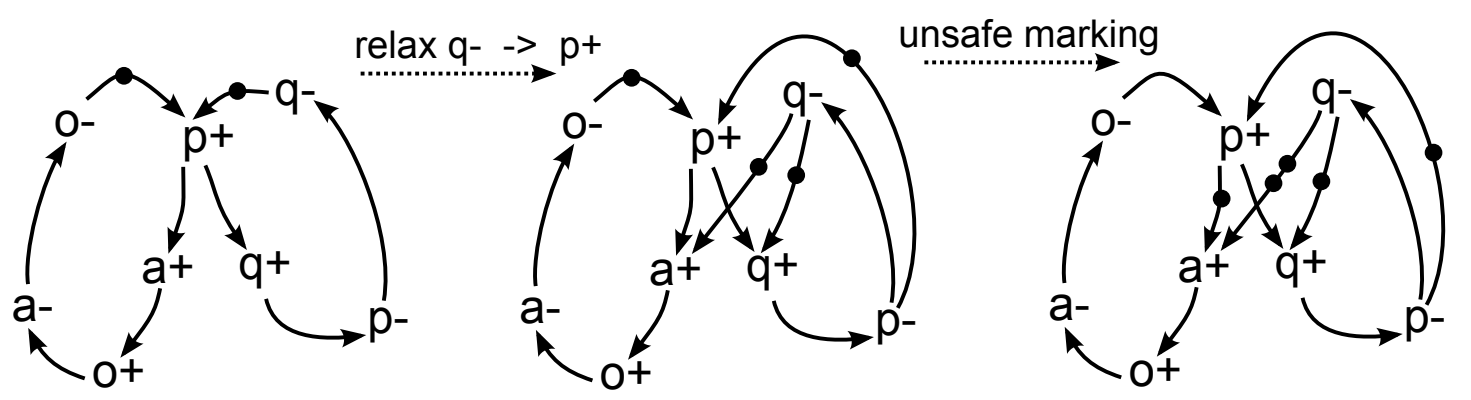

Figure 5.7: un-safeness caused by relaxation -
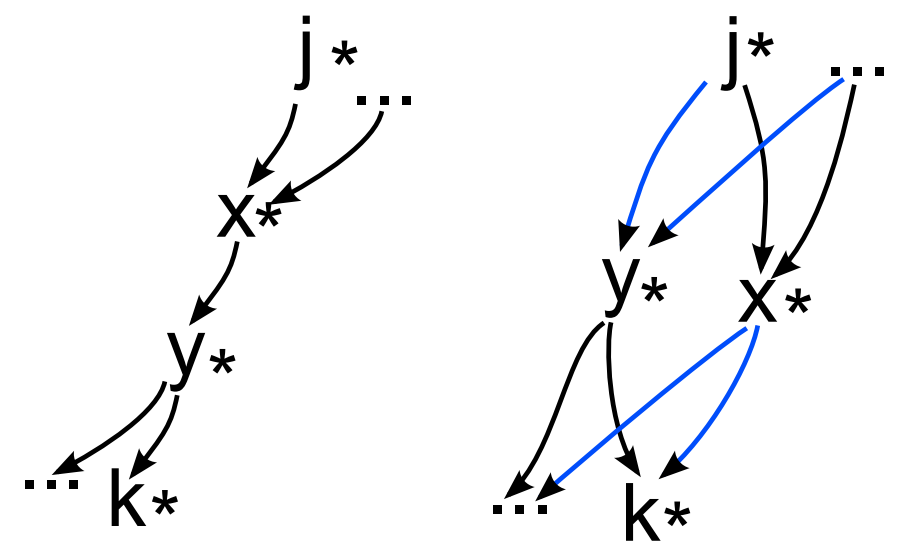

Figure 5.8: possible unsafe places after relaxation -

Lemma 2: The relaxation of an $\operatorname{arc} x * \Rightarrow y *$ in a live and safe MG component of a local STG does not influence safeness unless the gate has redundant literals, where redundant means that these literals could be removed without affecting the behavior of the gate.

Proof: 


\subsection{Timing ordering relaxation}

When an $\operatorname{arc} x * \Rightarrow y *$ in an MG is relaxed (as exemplified in Figure 5.8), only the newly added places $(\operatorname{arcs})<j *, y *>$ and $\left\langle x *, k *>\right.$ (where $j * \in^{\triangleleft} x *$, $\left.k * \in y *^{\triangleright}\right)$ could be unsafe.

If there is a transition $k * \in y *^{\triangleright}$ such that the place $\langle x *, k *>$ is unsafe then it must be the case that the transition $x *$ could fire twice without firing transition $k *$. If this is the case, the original MG cannot have a cycle $c y c=x * \Rightarrow y * \Rightarrow$ $k * \Rightarrow \sigma \Rightarrow x *$ otherwise transition $x *$ cannot fire twice without the firing of $k *$. Moreover, because the place $\langle x *, y *>$ is safe in the original MG, there must be a cycle $c y c_{1}=x * \Rightarrow y * \Rightarrow \sigma^{\prime} \Rightarrow x *$, in the MG and for the same reason it must be a cycle $c y c_{2}=y * \Rightarrow k * \Rightarrow \sigma^{\prime \prime} \Rightarrow y *$, in the MG. The two cycles $c y c_{1}$ and $c y c_{2}$ cannot have common vertex other than $y *$, otherwise, suppose they have another common vertex $l *$ then one cycle $c y c_{3}=x * \Rightarrow y * \Rightarrow k * \Rightarrow l * \Rightarrow \cdots \Rightarrow x *$ exists in the MG, which contradicts that the MG cannot have a cycle $c y c=x * \Rightarrow y * \Rightarrow$ $k * \Rightarrow \sigma \Rightarrow x *$.This is shown in Figure 5.9.

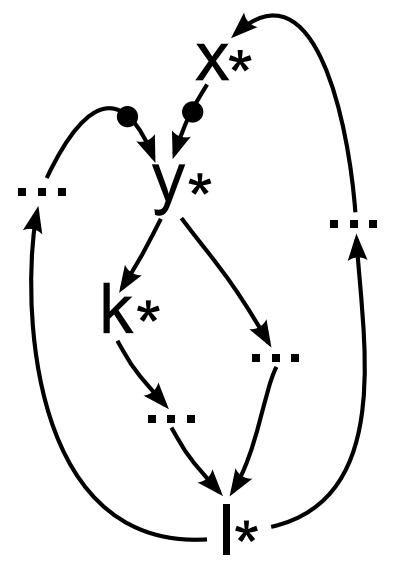

Figure 5.9: If two cycles have a common vertex $l$ -

The only possible shape of the MG that would have an unsafe place < 


\subsection{Timing ordering relaxation}

$x *, k *>, k * \in y *^{\triangleright}$ after relaxing the arc $x * \Rightarrow y *$ is shown in Figure 5.10. After relaxing the arc $x * \Rightarrow y *, \forall k * \in y *^{\triangleright}$ such that there is no cycle which contains both $x *$ and $k *$, the newly added place $\langle x *, k *>$ will be unsafe. This is because that transition $x *$ could continuously fire twice without firing $k *$, so if the arc $x * \Rightarrow k *$ was explicitly added, the corresponding place $\langle x *, k *>$ will be unsafe. There will be one cycle containing all transitions on the signal $x$ and one cycle containing all transitions on the signal $k$. Otherwise, two transitions $x *$ and $x *(k *$ and $k *$ ) will be concurrent, which violates the consistency property of the STG. Without loss of generality, assume that $c y c_{1}=x * \Rightarrow y * \Rightarrow \sigma^{\prime} \Rightarrow x *$ is the cycle that contains all the transitions on the signal $x$ and $c y c_{2}=y * \Rightarrow k * \Rightarrow \sigma^{\prime \prime} \Rightarrow y *$ is the cycle that contains all the transitions on the signal $k$ (as the two cycles in figure5.10). Due to our relaxation rule, only the arc between two input events could be relaxed, the signal $y$ cannot be the output signal of the gate ( $y$ must be a input variable). At least one cycle between $c y c_{1}$ and $c y c_{2}$ does not contain any transition on the output signal $o$; otherwise there will be two transitions $O *$ and o* appear at two cycles respectively. The consistency property of the STG will be violated. It has been proved that $y *$ is the only common vertex of two cycles, this indicates that all the transitions on $x$ or $k$ are totally concurrent with all the transitions on the output signal $o$. Without loss of generality, assume that all the transitions on signal $x$ are concurrent with all the transitions on signal $o$. The transitions on $x$ could fire freely, without any limitation to the firing of transitions on $o$. This indicates that the value of $o$ does not depend on the value of $x$. So, $x$ is redundant.

By the same logic, the Figure 5.11 shows the only possible shape of the MG 
that would have an unsafe place $\left\langle j *, y *>, j * \in^{\triangleleft} x *\right.$ after relaxing the arc $x * \Rightarrow y *$. The transition $x *$ should be the only common vertex of the two cycles containing $j *$ and $y *$, transition $j *$ could fire twice without firing transition $y *$. At least one signal between $y$ and $j$ is redundant.

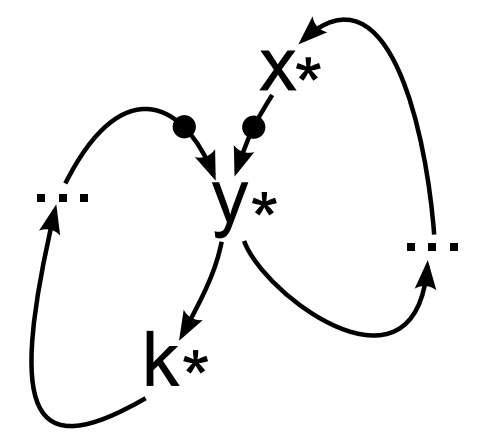

Figure 5.10: Shape of MG that will have an unsafe place $<x *, k *>$, $k * \in y *^{\triangleright}$ after relaxing $x * \Rightarrow y *-$

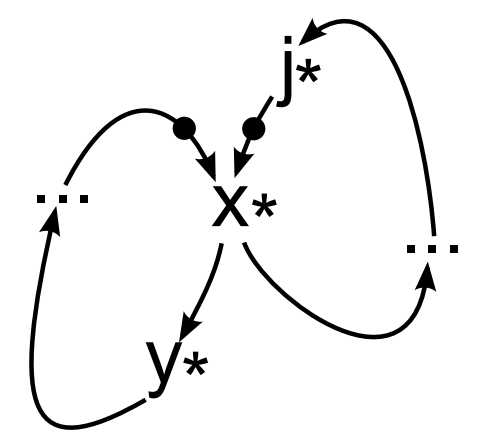

Figure 5.11: Shape of the MG that will have an unsafe place $<j *, y *>$, $j * \in^{\triangleleft} x *$ after relaxing $x * \Rightarrow y *-$

The top two diagrams in Figure 5.12 show the gate $o$, which contains a redundant literal $p$, together with its local STG. The literal $p$ is redundant because the only cube $c_{1}=b p$ that contains the literal $p$ is covered by another cube $c_{2}=b$, 


\subsection{Timing ordering relaxation}

we have $c_{1} \sqsubseteq c_{2}$. The literal $p$ is redundant in gate $o$, the value of gate $o$ will never depend on the literal $p$. So, other input signals and the output signal of gate $o$ could transition more than once without waiting for any transitions on signal $p$. In this situation, if we do the relaxation on arc $p-\Rightarrow b+$ on the STG, the resulting STG will not be safe. When the redundant literal $p$ is removed from the gate and from its local STG, as was shown at the bottom two diagrams in Figure 5.12, relaxation will not break the safeness on the resulting STG.
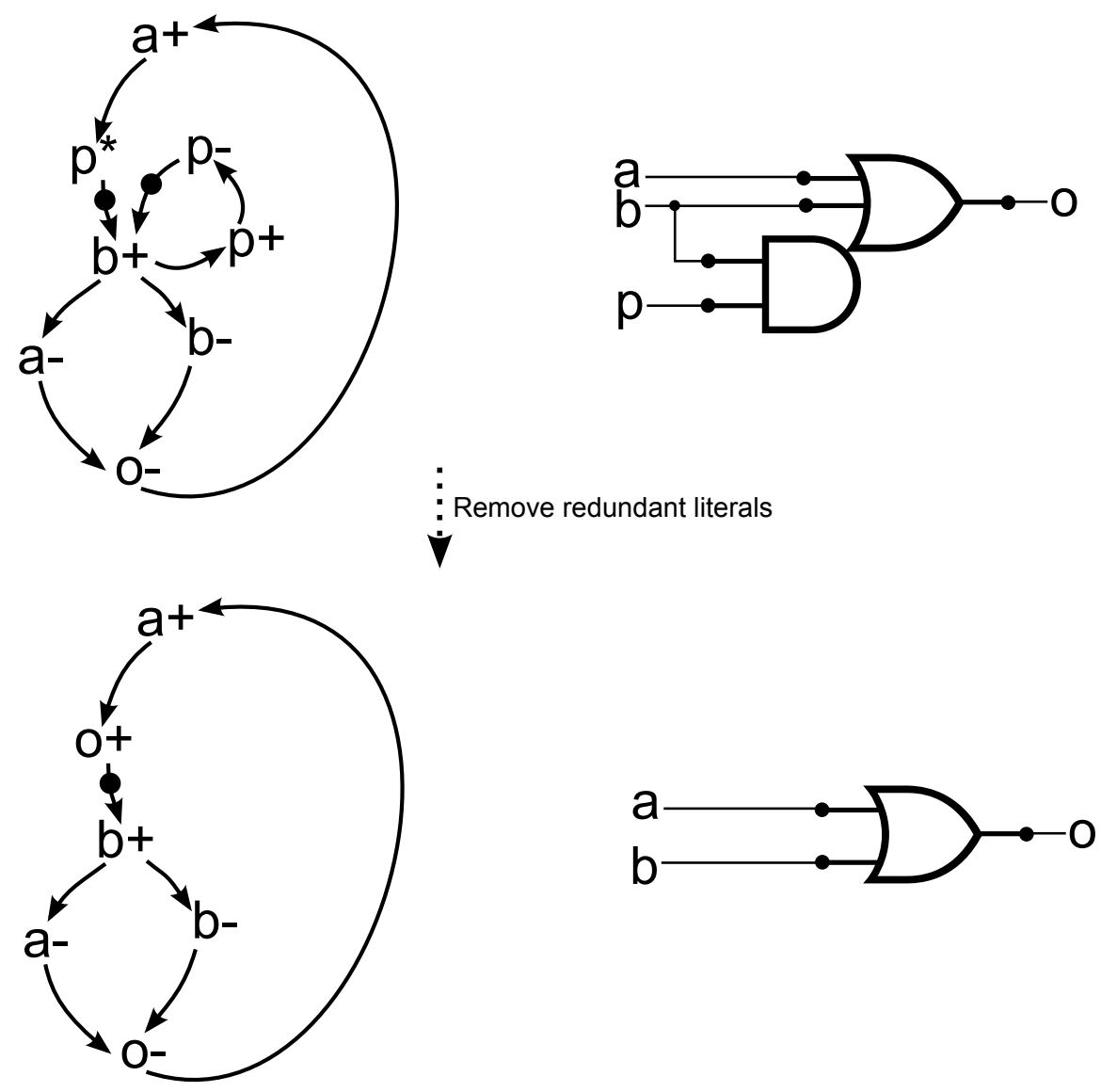

Figure 5.12: Gate $o$ has a redundant literal $p$ 


\subsubsection{Removing redundant arcs}

The relaxation of a single arc will add a set of new arcs into the STG. Some of the newly added arcs could be redundant. An arc is redundant ${ }^{1}$ if the order constraints imposed by this arc has been guaranteed by other arcs. E.g. in the Figure 5.13, after relaxing the $\operatorname{arcs} b+\Rightarrow a-$, two arcs $o+\Rightarrow a-$ and $b+\Rightarrow o-$ will be added, where the arc $o+\Rightarrow a-$ is a redundant arc. The $\operatorname{arcs} b+\Rightarrow b-\Rightarrow$ $o-$ have already constrained that $b+$ would happen before $o-$.

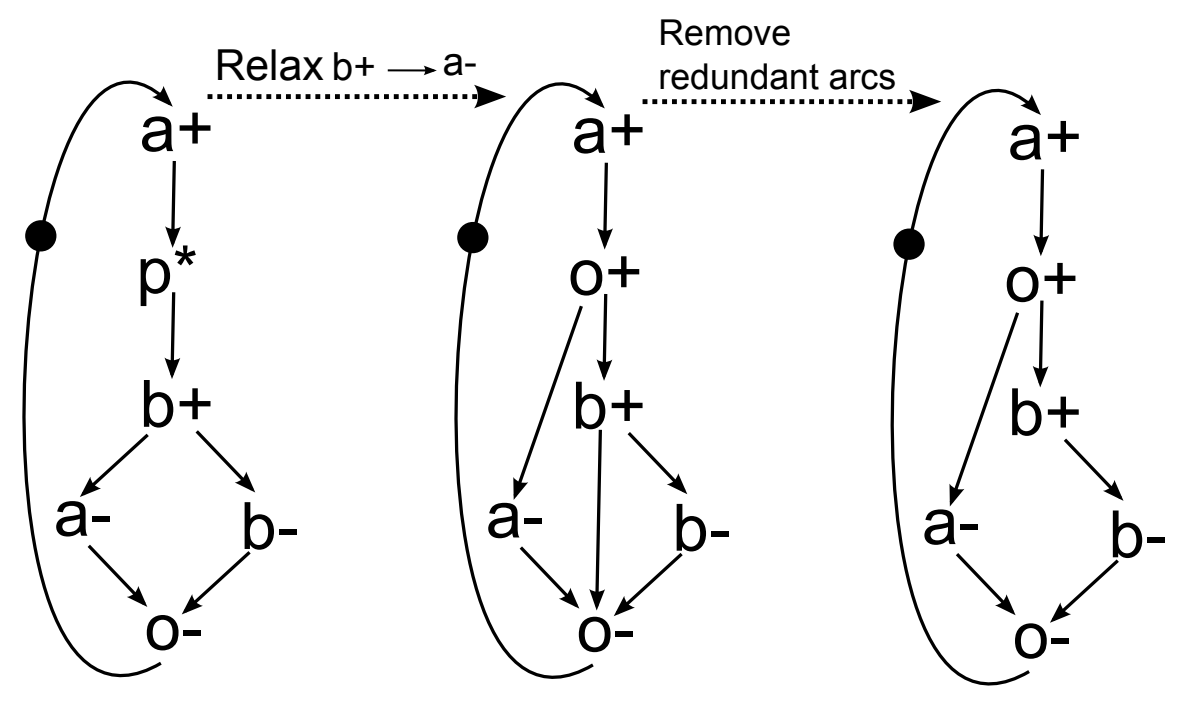

Figure 5.13: redundant arcs due to the relaxation -

Redundant arcs do not contribute to the flow of an STG, but their existence might complicate the relaxation and analysis processes. So, redundant arcs should be detected and removed after new arcs are added into the STG.

\footnotetext{
${ }^{1}$ In this thesis, the term redundant is used to characterize the arcs and places which could be eliminated without affecting the flow sequences in the underlying STG. In other literature, e.g. [61], different terms (implicit place) might be used. Due to the fact, the redundancy and implicitness is equivalent w.r.t. places in a live MG, the term redundant is used to generalize this characteristic. The term implicit are used to characterize the un-drawn places in an MG.
} 


\subsection{Timing ordering relaxation}

Please note that for each arc $x * \Rightarrow y *$ in the local STG, there is an implicit place denoted by $\langle x *, y *\rangle$ on it. An arc is redundant means that the corresponding place $\langle x *, y *\rangle$ and the flow relation w.r.t this place could be eliminated without affecting the flow sequences in the original STG. A redundant place will never disable the firing of one transition on its own [62]. This suggests that the redundant place could be checked in an $\mathrm{MG} N=\left(P, T, F, m_{0}\right)$ whose marking set is $\mathfrak{M}$ as follows,

A place $p$ is redundant if $\forall m \in \mathfrak{M}, m(p)=0 \Rightarrow \exists p^{\prime} \in P \wedge p^{\prime} \neq p \wedge p^{\prime} \in \bullet^{\bullet}\left((p)^{\bullet}\right) \wedge$ $m\left(p^{\prime}\right)=0$

However, this requires to generate the marking set of an STG and check each marking in it. [61] suggests a method to check the redundancy of a place in an MG structurally, which avoids the generation of marking set.

In [61], researchers had proved that in a live MG, a redundant place would either be a loop-only place or a shortcut place.

In a live MG, a place $p$ is a loop-only place if $\bullet p=p^{\bullet} \wedge m_{0}(p)=1$; a place $p$ is a shortcut place if there is a path $\sigma=\bullet p \cdots p^{\bullet} \wedge p \notin \sigma \wedge \sum_{p^{\prime} \in \sigma} m_{0}\left(p^{\prime}\right) \leq m_{0}(p)$.

The place $p_{4}$ in the Figure $5.14(\mathrm{a})$ is a shortcut place, because there is a path $\sigma=\left(x+, p_{2}, y+, p_{3}, x-\right)$ between two transitions $\bullet p_{4}=x+$ and $p_{4}^{\bullet}=x-$ and $m_{0}\left(p_{4}\right) \geq \sum_{p^{\prime} \in \sigma} m_{0}\left(p^{\prime}\right)=m_{0}\left(p_{2}\right)+m_{0}\left(p_{3}\right)=0$.

However, the place $p_{11}$ in Figure 5.14 (b) is not a shortcut place, the path connecting two transitions ${ }^{\bullet} p_{11}=b-$ and $p_{11}^{\bullet}=b+$, which contains the least tokens, is shown by the dotted line in the graph. The total number of tokens on this path is two which is greater than the number of tokens in place $p_{11}$.

In order to check the redundancy of a place $p_{0}$, one needs to check whether 


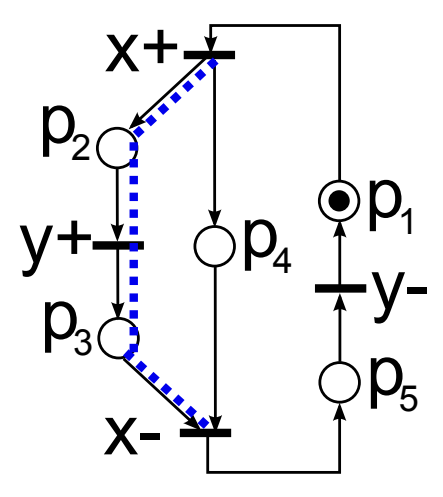

(a)

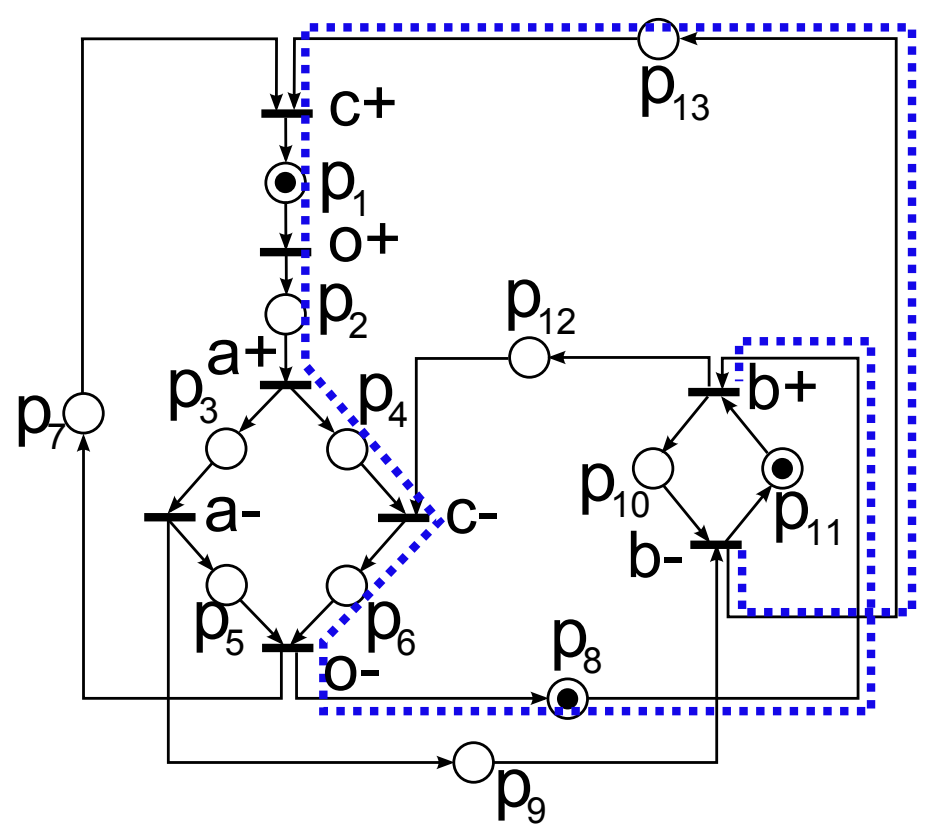

(b)

Figure 5.14: Property of shortcut places - 


\subsection{Timing ordering relaxation}

there exists one path from ${ }^{\bullet} p_{0}$ to $p_{0}^{\bullet}$ and the number of tokens on this path should be less than or equal to the number of tokens in $p_{0}$. This could be done by changing the MG into an edge weighted directed graph and searching the shortest path between two nodes ${ }^{\bullet} p_{0}$ and $p_{0}^{\bullet}$ as was proposed in [61].

Given a marked graph $M G=\left(T, P, F, m_{0}\right)$, a directed graph $G=(V, E)$ is built by creating one node $t$ for each transition $t * \in T$ in $M G$ and creating one edge $e=\left(\bullet p, p^{\bullet}\right)$ for each place $p \in P \wedge p \neq p_{0}$. The weight of each edge $e=\left(\bullet p, p^{\bullet}\right)$ is the number of tokens in the place $p$ in initial marking $m_{0}$. The edge weights in the directed graph $G=(V, E)$ are non-negative and the shortest path between two nodes could be solved by Dijkstra algorithm. A pseudo-code for checking the redundancy of a place $p_{0}$ in the marked graph $M G$ is shown in Algorithm 3. The time complexity of this algorithm is $O\left(n^{2}\right)$, where $n$ is the number of transitions in the MG.

The directed graphs corresponding to the MGs in Figure 5.14, which are used to check the redundancy of the place $p_{0}$, is shown in Figure 5.15. The dotted arc corresponds to the place in the original MG for checking redundancy and. In graph (a) in Figure 5.15, the shortest path from $x+$ to $x-$ is $x+, y+, x-$ whose weight is 0 , equal to the number of token in the place $<x+, x->$. So, the place $<x+, x->$ is redundant in the original MG. However, in graph (b), the shortest path from $b-$ to $b+$ is $b-, c+, o+, a+, a-, o-, b+$ whose weight is 2 , larger than the number of token in the place $<b-, b+>$. So, the place $<b-, b+>$ is not redundant. 

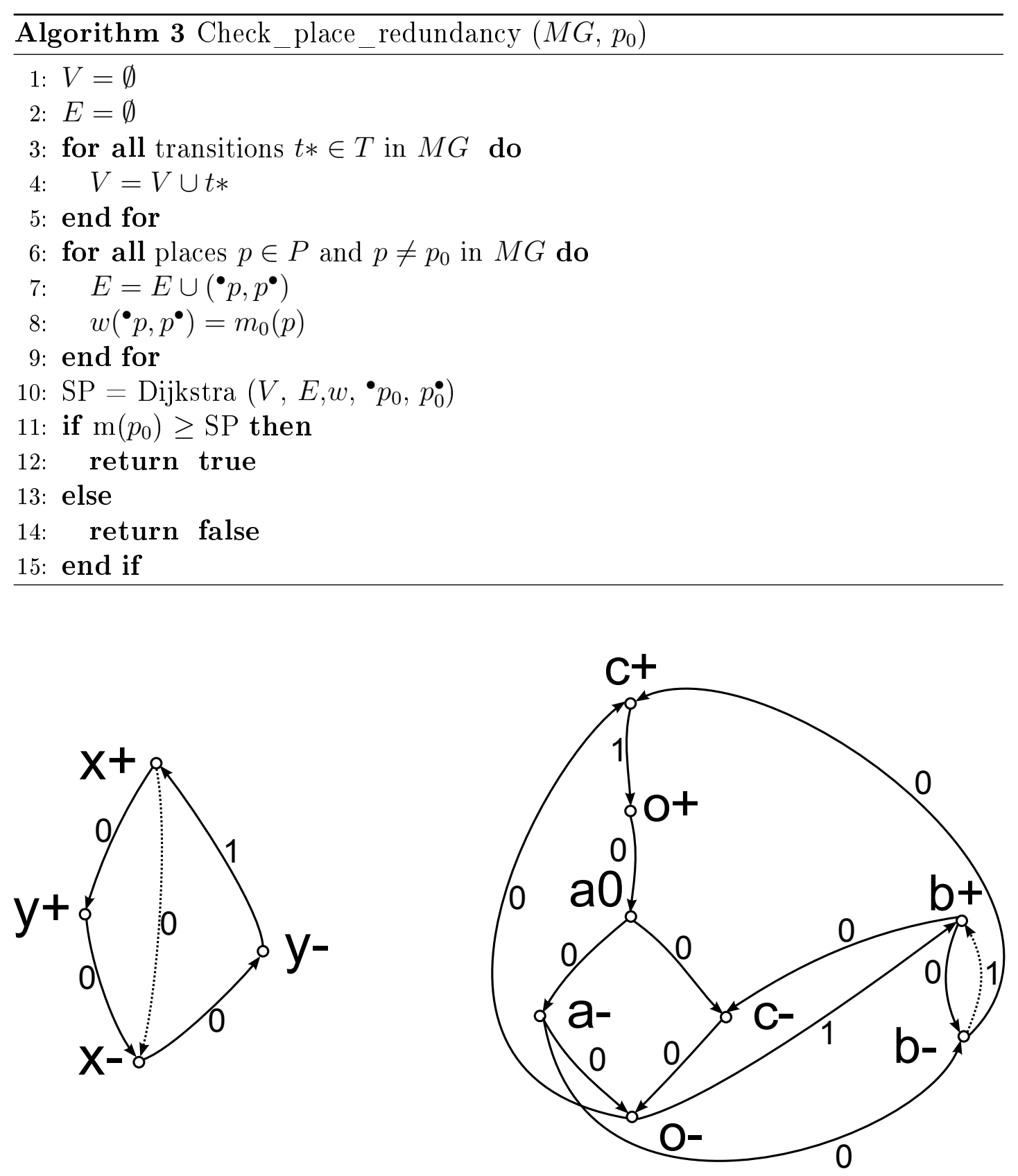

(a)

(b)

Figure 5.15: Check for shortcut places using Dijkstra's algorithm - 


\subsection{Local STG relaxation and hazard criterion}

\subsubsection{Four relaxation cases}

The relaxation of one arc in the STG, which will make two ordered transitions into concurrent ones, will expand the reachable states of the STG. Whether this relaxation is acceptable or not depends on whether the newly introduced states cause glitches. A glitch is a premature transition, in which the output of a gate is enabled to fire when it is expected to remain stable.

Timing conformance: A local STG of a gate $o$, is said to be in timing conformance to gate $o$ if in the SG of the STG: $\left\{f_{o \uparrow}(s)=1 \mid s \in E R(o+) \vee s \in Q R(o+)\right\}$ and $\left\{f_{o \downarrow}(s)=1 \mid s \in E R(o-) \vee s \in Q R(o-)\right\}$.

The timing conformance of a local STG with respect to a gate is that, the output value of this gate should evaluated to "1" at each state in $E R(o+)$ and $Q R(o+)$ and should evaluated to "0" at each state in $E R\left(o_{-}\right)$and $Q R\left(o_{-}\right)$in the SG of STG.

The timing conformance of a local STG to a gate $o=a \cdot b$ is illustrated in Figure 5.16, where diagram (a) and diagram (b) show the gate and its initial local STG and the corresponding SG. Diagram (c) shows the STG, where the arc $a+\Rightarrow b+$ is relaxed, and the corresponding SG. In the SG of the resulting STG, gate $o$ evaluates to "1" in every state in $E R(o+)$ and $Q R(o+)$ and evaluates to "0" in every state in $E R(o-)$ and $Q R(o-)$. So, the STG in diagram (c) is in timing conformance to the gate $o$. However, the STG in diagram (d), which is derived by relaxing the arc $b-\Rightarrow a+$ in diagram (b), is not in timing conformance to the gate $o$. Because there is a state $a b o=110$ in $Q R(o-)$ at which the $f_{o \uparrow}=a b$ 


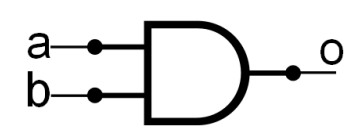

(a)
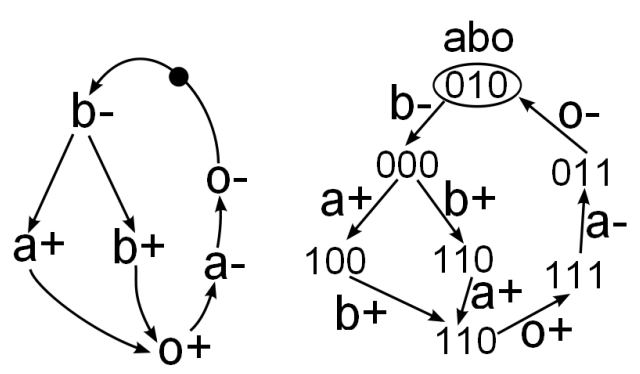

(c)
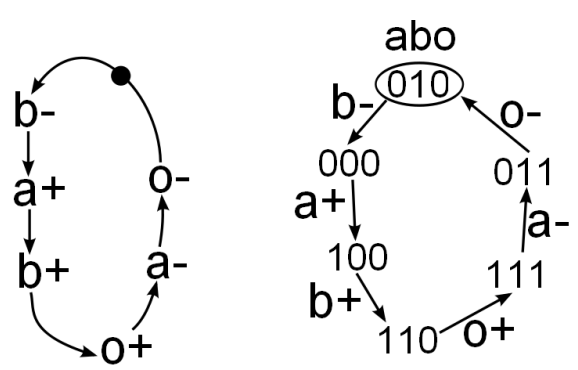

(b)

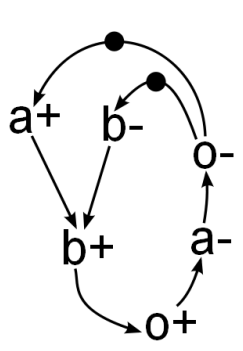

$\mathrm{QR}(0-)$ abo

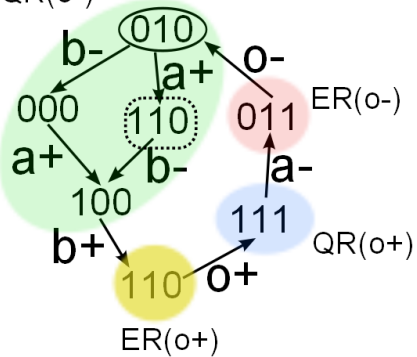

(d)

Figure 5.16: Illustration of timing conformance. (a) the gate and (b)-(d) local STGs and the corresponding SGs - 
evaluates to "1" and the gate would prematurely fire to " 1 " without waiting for the transition $b+$ to come.

After relaxing one arc $x * \Rightarrow y *$, if the resulting STG is in timing conformance to the gate, it indicates that the gate will be glitch-free even when the transition $x *$ comes later than transition $y *$. But the reverse is NOT always true. If the resulting STG is not in timing conformance to the gate, the gate still could be glitch-free if the violation of timing conformance is due to unnecessary relation constraints or the so-called "OR-causality" [63] [64].

The prerequisite transition set of the $i$-th occurrence of an output $o *$ is defined as $E_{\text {pre }}(o * / i)=\left\{z *: z * \in\left({ }^{\triangleleft} O * / i\right)\right\}$. The output transition should only fire when all transitions in its prerequisite transition set have fired; otherwise, the firing of an output transition is a glitch. The prerequisite transition set for each transition on output signal $o$ is calculated before relaxation and is used to check the correctness after this relaxation has been carried out.

When an $\operatorname{arc} x * \Rightarrow y *$ belongs to the local STG of gate $o$, for the SG of the resulting STG, one and only one of the following four cases will happen.

Relaxation case 1: $\forall s \in Q R(o+), f_{o \downarrow}(s)=F A L S E$ and $\forall s \in Q R(o-)$, $f_{o \uparrow}(s)=F A L S E$.
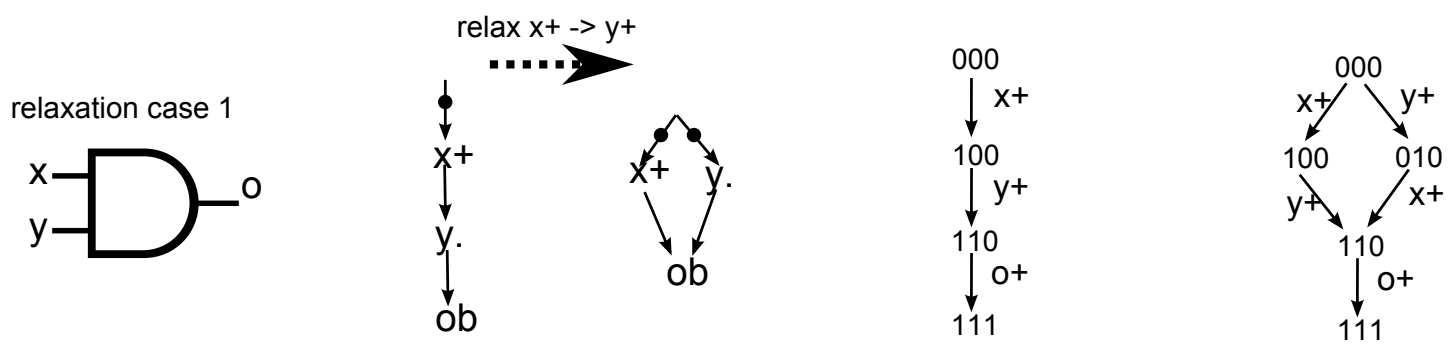

Figure 5.17: Relaxation case 1 - 
Relaxation case 1 relates to the situation that the resulting STG is in timing conformance to the gate. If so, the gate is guaranteed to be glitch-free under the new STG.

Relaxation case 2: $\exists s \in Q R(o+)$ such that $f_{o \downarrow}(s)=T R U E$ and $\forall s \in$ $Q R_{i}(o+)$ such that $f_{o \downarrow}(s)=T R U E, s(z)=1$ if $z+\in E_{\text {pre }}(o-/ j) ; s(z)=0$ if $z-\in E_{\text {pre }}(o-/ j)$, where $Q R_{i}(o+)$ is followed by $E R_{j}(o-)$. Or similarly for $Q R(o-), f_{o \uparrow}, Q R_{i}(o-)$ and $E R_{j}(o+)$.
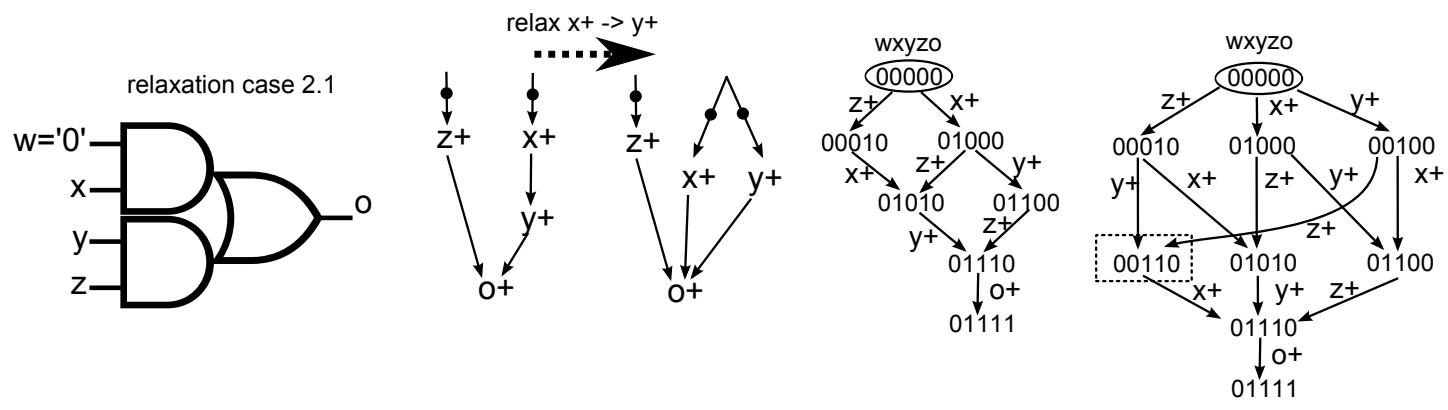

Figure 5.18: Relaxation case 2 -

Relaxation case 2 relates to the situation that the gate evaluates to "0" ("1") in some states in $Q R(o+)(Q R(o-))$, but in all these states, all the prerequisite transitions have fired. Even if the resulting STG violated the timing conformance, this situation does not indicate a glitch. This situation occurs when a transition, which cannot be acknowledged by an output transition, is unnecessarily put into the prerequisite set of an output transition.

The Figure 5.18 shows a gate $o$ with its STG segment. The literal $w$ remains at "0" during this segment. When the arc $x+\Rightarrow y+$ is relaxed, the transition $x+$ is set to be one of the prerequisite events of $o+$. However, due to the gate function, the occurrence of $x+$ cannot be acknowledged by $o+$, thus the gate becomes 
enabled to fire $o+$ in state '00110' (as is indicated by the dashed square in the figure) in $Q R(o-)$. This is not a premature firing, because all the transitions $\{z+, y+\}$ in the prerequisite set of $o+$ in the STG before relaxing have fired; the $o+$ is allowed to be fired.

Relaxation case 3: $\exists s \in Q R_{i}(o+)$ such that $f_{o \downarrow}(s)=T R U E$ and either $x+\in E_{\text {pre }}(o-/ j)$ and $s(x)=0$ or $x-\in E_{\text {pre }}(o-/ j)$ and $s(x)=1$, where $Q R_{i}(o+)$ is followed by $E R_{j}(o-)$. For all $s$ that fulfills all conditions above, $x *$ is excited in $s$ and $s^{\prime}$ is the state obtained by firing $x *$ in $s, s^{\prime} \in E R_{j}(a-)$. Or similarly for $Q R(o-), f_{o \uparrow}, Q R_{i}(o-)$ and $E R_{j}(o+)$.
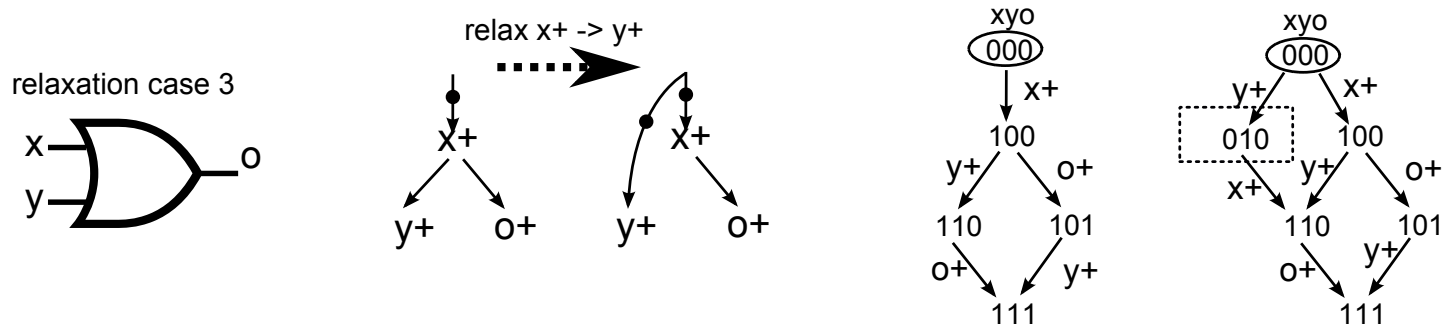

Figure 5.19: Relaxation case 3 -

Relaxation case 3 applies to the situation when the gate evaluates to "0" ("1") in some states in $Q R(o+)(Q R(o-))$, and for all these states, $x *$ is excited and the states reached by firing $x *$ is in $E R(o-)(E R(o+))$. Relaxation case 3 is still not a hazardous situation. Because in all states in $Q R(o+)$ or $Q R(o-)$ where the gate is ready to transit, $x *$ is the only transition that belongs to the prerequisite set of $o+$ or $o-$ that has not fired yet. The reason that the gate becomes excited without waiting for transition $x *$ after relaxing the arc $x * \Rightarrow y *$ is that transition $y *$ could make another clause in $f_{o \uparrow}$ or $f_{o \downarrow}$ become true. This is called OR-causality in [63] and [64]. This is not a hazard because the arc $x * \Rightarrow y *$ 
indicates that (in gate $y$ ) $x *$ is acknowledged by $y *$, so when $y *$ arrives, $x *$ must have occurred (even though $x *$ has not propagated to gate $o$ yet).

The Figure 5.19 shows a gate $o$ with its STG segment. When the arc $x+\Rightarrow y+$ is relaxed, the gate could reach a state '010' in $Q R(o-)$, in which $x+$ is enabled. By firing $x+$ in state '010' a new state '110', which is in $E R(o+)$, is reached. This corresponds to the relaxation case 3 .

Relaxation case 4: $\exists s \in Q R_{i}(o+), f_{o \downarrow}(s)=T R U E$ and either $\exists z+\epsilon$ $E_{\text {pre }}(o-/ j)$ and $s(z)=0$ or $\exists z-\in E_{\text {pre }}(o-/ j)$ and $s(z)=1, s^{\prime}$ is the state by complementing the value of signal $x$ in $s$ and $s^{\prime} \notin E R_{j}(o-)$ where $Q R_{i}(o+)$ is followed by $E R_{j}(o-)$. Or similarly for $Q R(o-), f_{o \uparrow}, Q R_{i}(o-)$ and $E R_{j}(o+)$.
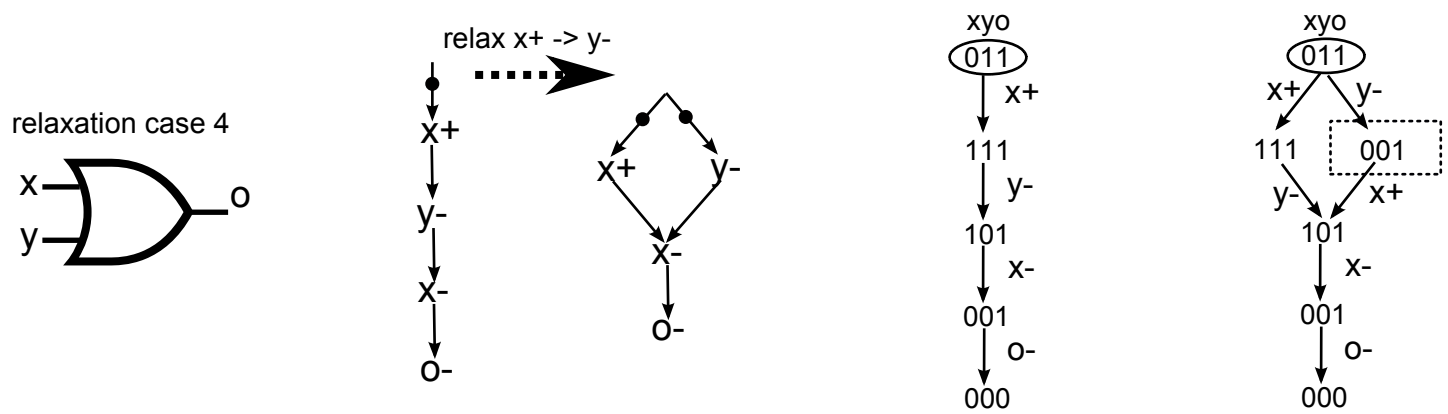

Figure 5.20: Relaxation case 4 -

Relaxation case 4 applies to the situation when an arc $x * \Rightarrow y *$ is relaxed, the gate becomes enabled in some states in $Q R(o+)$ in $Q R(o-)$ in the resulting SG. For at least one of these states, not all the transitions in prerequisite set of $o-$ or $o+$ have fired and the state derived by complementing the $x$ value is not in $E R(o-)$ or $E R(o+)$.

In Figure 5.20, when the arc $x+\Rightarrow y-$ is relaxed, the gate could reach a state '001' in $Q R(o+)$, in which the gate is enabled to transit to "0" and by 


\subsection{Local STG relaxation and hazard criterion}

complementing the value of $x$ in state '001', the newly reach state '101' is still in $Q R(o+)$. The relaxation case 4 is equal to that in the "problematic" state, there exists another transition in the prerequisite set of $o+$ or $o-$ other than $x *$, which has not fired yet.

The relaxation case 1 indicates the relaxation of arc $x * \Rightarrow y *$ will be accepted immediately. The STG will be updated for further operation. The relaxation case 4 indicates the relaxation will be rejected and one timing constraint $x * \prec y *$ (which means that $x *$ must reach the gate before $y *$ ) will be generated to prevent the gate from entering the hazardous states. The relaxation cases 2 and 3 requires further modifications of the STG.

The relaxation case 2 happens when a transition $x *$, which is not necessary for the firing of the output transition $o *$, is relaxed into one of prerequisite transitions of $o *$. The gate $o$ could become enabled without waiting for the occurrence of $x *$, so the gate becomes excited in some states in $Q R(o *)$. In this case, $x *$ should be concurrent with $o *$, which is done by relaxing the arc $x * \Rightarrow o *$. There could be two cases after making $x *$ to be concurrent with $O *$.

The first case is exemplified in Figure 5.21 (a), where after making $x+$ to be concurrent with $o+, f_{o \uparrow}$ is true in every state in $E R(o+)$. However, in the second case, there exists one state $s \in E R(o+)$ such that $f_{o \uparrow}$ is false in $s$. One example for the second case is shown in Figure 5.21 (b), $x+$ is not necessary for $o+$, because there is another transition $z+$ which is concurrent with $o+$ and if $z+$ reaches gate $o$ earlier than $x+$, it will trigger $o+$ instead of $x+$. This means that neither $z+$ nor $x+$ is necessary for $o+$, neither of them could be a prerequisite transition of $o+$. So, when $x+$ is relaxed to be one of prerequisite transition of 

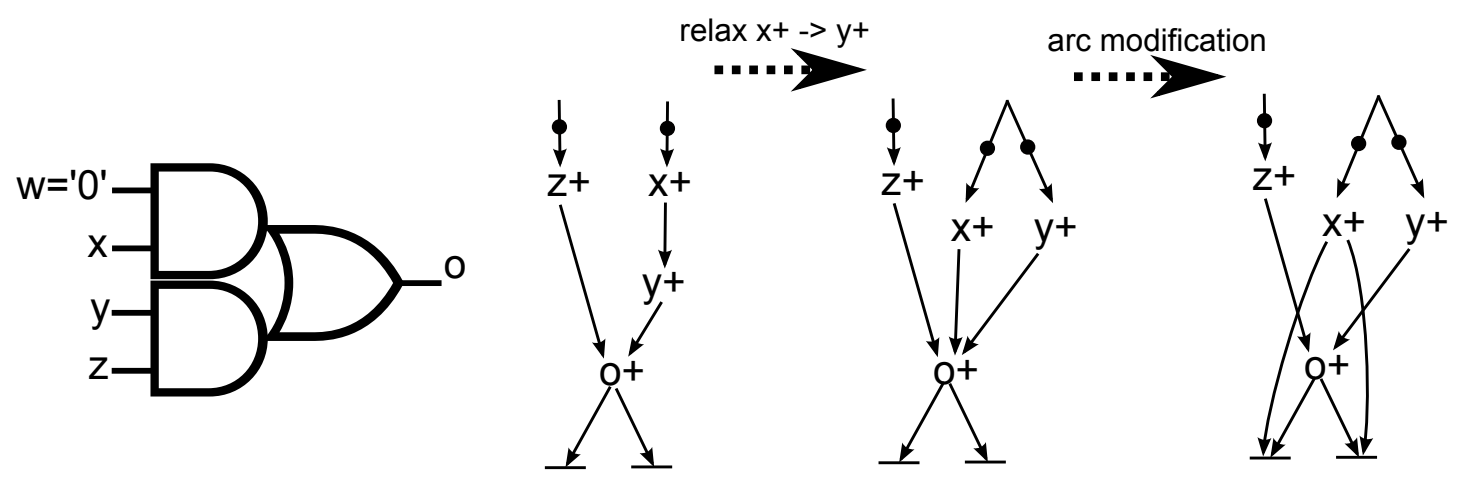

(a)

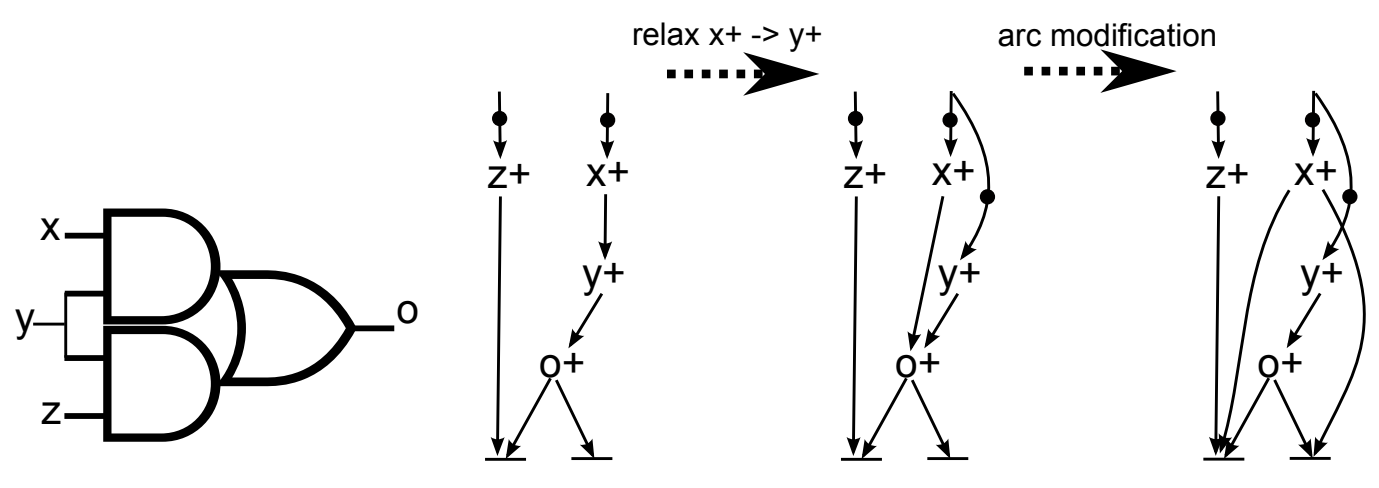

(b)

Figure 5.21: Arc modification in case 2. (a) without OR-causality and (b) with OR-causality. - 


\subsection{Optimal relaxation order}

$o+$, it must be made to be concurrent with $o+$ as discussed above. However, when $x+$ is modified to be concurrent with $o+$, the $E R(o+)$ could be entered by just firing $y+$ in the initial marking, where $f_{o \uparrow}$ is still false. This happens because the enabling of gate $o$ needs at least one transition between $x+$ and $z+$ to happen first, but neither of them are necessary for $o+$, when both of them are concurrent with $o+$, the $E R(o+)$ could be entered when $f_{o \uparrow}$ is still false. This problem is caused by the OR-causality, where the enabling of a gate could be caused by more than one option. Both relaxation case 2 and case 3 could meet the OR-causality. The details of OR-causality situation and decomposition of the OR-causality will be specified in detail in Chapter 6 .

\subsection{Optimal relaxation order}

Different relative timing constraint sets might be derived if arcs are relaxed in different orders. This is because there might be more than one way to prevent a gate entering a hazardous state.

The diagram (a) in Figure 5.22 show an STG segment whose SG (shown in diagram (b)) contains a hazardous state $s$. There are two approaches to forbid the circuit to visit this hazardous state (timing constraints are denoted by \&): 1) to force $c+$ before $a+$ (the corresponding STG segment and SG are shown in diagram $(\mathrm{c})$ and $(\mathrm{d})) ; 2$ ) to force $b+$ to come before $a+$ (the corresponding STG segment and SG are shown in diagram (e) and (f)).

One example which shows different timing constraints due to different relaxation orderings is presented in Figure 5.23. Four different sets of timing con- 


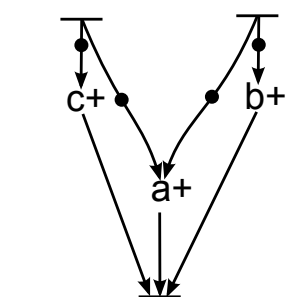

(a)

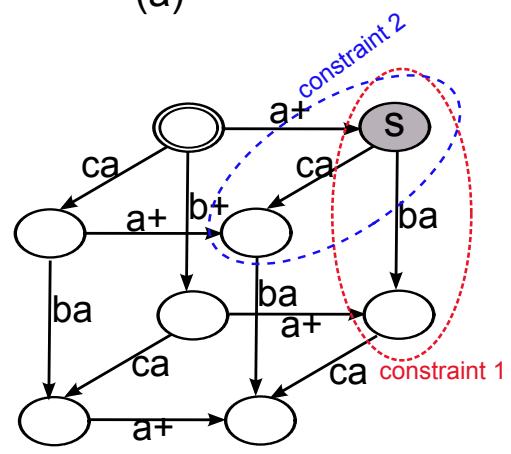

(b)

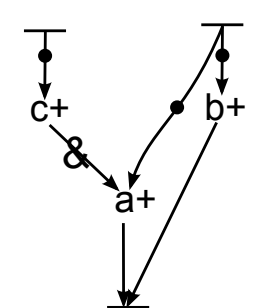

(c)

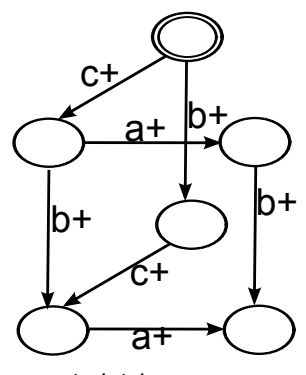

(d)

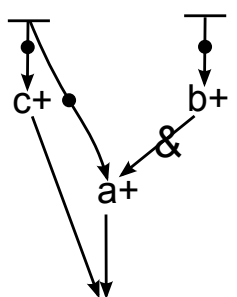

$\frac{1}{(e)}$

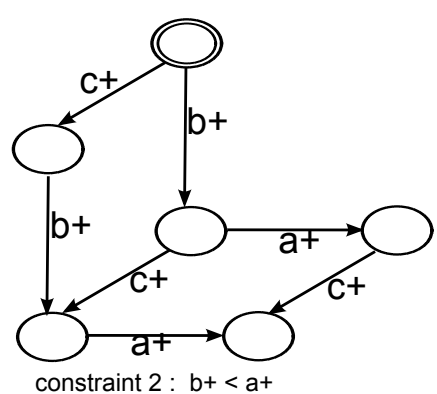

(f)

Figure 5.22: Preventing the gate entering hazardous state $s$ -

straints could guarantee the correctness of the circuit:

1) $\{x+\prec y-, y+\prec z+\}$ (relax $x+\Rightarrow z+$ before $y+\Rightarrow z+$ and then relax $y+\Rightarrow x-$ before $x+\Rightarrow y-)$;

2) $\{y+\prec x-, y+\prec z+\}($ relax $x+\Rightarrow z+$ before $y+\Rightarrow z+$ and then relax $x+\Rightarrow y-$ before $y+\Rightarrow x-)$;

3) $\{x+\prec z+, y+\prec x-\}$ (relax $y+\Rightarrow z+$ before $x+\Rightarrow z+$ and then relax $x+\Rightarrow y-$ before $y+\Rightarrow x-)$ and

4) $\{x+\prec z+, x+\prec y-\}$ (relax $y+\Rightarrow z+$ before $x+\Rightarrow z+$ and then relax $y+\Rightarrow x-$ before $x+\Rightarrow y-)$.

We prefer to generate the optimal one during the relaxation process rather than generate all of the cases and choose the best one. Exhaustion of all relaxation orders implies a time complexity of $O(n !)$, where $n$ is the number of arcs to be 
relaxed, and most of these relaxations lead to the same results.

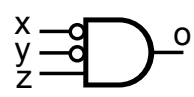

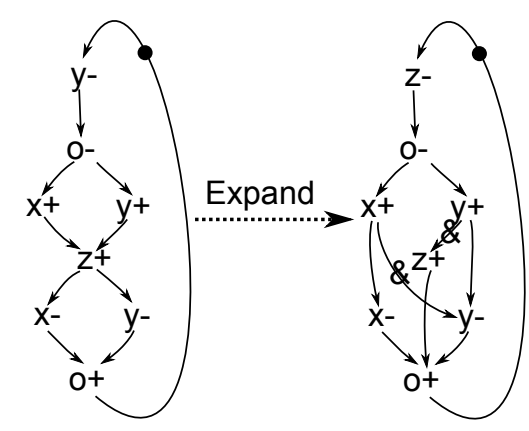

1)

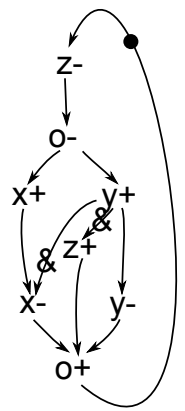

2)

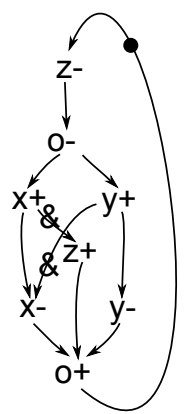

3)

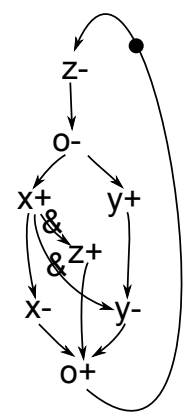

4)

Figure 5.23: Different timing constraints due to different relaxation ordering

The weakest constraint set could be generated by relaxing the tightest arc at each step. This will relax tighter arcs as much as possible before they become the necessary timing requirement to avoid entering the hazardous state. In Figure 5.24, when deciding which of the two arcs $c+\Rightarrow a+$ and $b+\Rightarrow a+$ should be relaxed first, their weights (the level of adversary path) could be calculated from the $S T G_{i m p}$. The $S T G_{i m p}$ explicitly presents adversary paths; for example, violation of the arc $c+\Rightarrow a+$ needs two adversary paths $c+\Rightarrow p-\Rightarrow q+\Rightarrow h-\Rightarrow a+$ and $c+\Rightarrow m-\Rightarrow n+\Rightarrow a+$ to be faster than the wire between fan-out of gate $c$ and fan-in of gate $a$. If all the signals $p, q, h, m, n, k, l$ are internal signals, the weight of $\operatorname{arc} c+\Rightarrow a+$ is three and that of $\operatorname{arc} b+\Rightarrow a+$ is two. The arc $b+\Rightarrow a+$ is tighter than arc $c+\Rightarrow a+$ and should be relaxed first. If there are several arcs of the same tightness, one arc will be picked randomly. The function find_tightest_arc $(S T G)$ returns the tightest input to input arc in a local STG of a gate, whose ordering has not been guaranteed yet. 


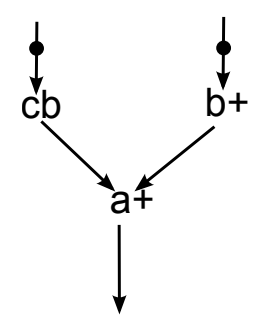

local STG

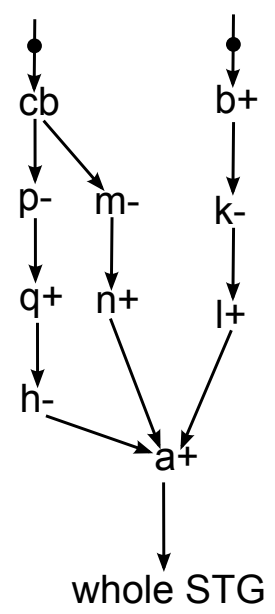

Figure 5.24: Calculate the weight of an arc from $S T G_{i m p}-$

\subsection{Top level algorithm}

The top level algorithm of the proposed method is shown in Algorithm 5, where the input STG will be decomposed into a set of MGs by the function Decompose_into_ $M G(S T G)$ using Hack's algorithm [8] if it contains free-choice places. Then the local STG for each internal (denoted by $R$ ) and primary output (denoted by $O$ ) signal $a$ will be generated by the function $\operatorname{Proj}(M G, a \cup \operatorname{fan}$-in(a)) introduced in Algorithm 1. The timing constraints for a gate $a$ to work correctly under an MG component will be derived in Algorithm 4 using the processes analyzed in the previous sections. The function Write_sg(STG) generates the SG of an STG using the method presented in [48]. Function $\operatorname{Relax}(S T G, x * \Rightarrow y *)$ is the arc relaxation algorithm defined in Algorithm 2. Function Check $\left(S G, f_{a \uparrow}\right.$, $\left.f_{a \downarrow}\right)$ decides which relaxation case will be due to the criteria introduced in this chapter. Lines 10-14 and 20-24 depict the OR-causality decomposition in relaxation case 2 and 3, which will be specified in detail in the next chapter. The 
timing constraint the transition $x *$ should arrive at gate $a$ before $y *$ is denoted as $a: x * \prec y *$ in line 28 . The timing constraints for a circuit to work correctly when the isochronic fork is relaxed is the union of the timing constraints of all gates under all MG components.

\subsubsection{Complexity analysis}

The time complexity of decomposing a free-choice STG into MGs using Hack's algorithm grows exponentially with respect to the number of choice places in an STG. However, the number of choice places in an STG is decided by the function of the circuit not the scale of the circuit. Usually, an STG does not contain a large number of choice places.

The complexity for deriving the local STGs for all gates in an SI circuit is $O\left(n^{6}\right)$ with respect to the number of transitions $(n)$ in the STG. In the worst case, there are $O(n)$ gates which need deriving their local STGs. There are up to $O(n)$ transitions needing to be eliminated for each local STG. When one transition is eliminated, each arc in the resulting STG needs to be checked for redundancy. The complexity for checking the redundancy of one arc is $O\left(n^{2}\right)$ and a graph which has $O(n)$ transitions could have up to $O\left(n^{2}\right)$ arcs.

When an STG is decomposed into a set of local STGs, checking hazards for all gates is performed on their local STGs. Thus, the complexity for the hazard checking work increases linearly with respect to the number of gates in the circuit.

So, the complexity of the proposed technique increases exponentially as the number of choice places in an STG. When the number of choice places in the 
STG is considered to be a constant, the asymptotic computation complexity is $O\left(n^{6}\right)$, where $n$ is the number of transitions in the STG.

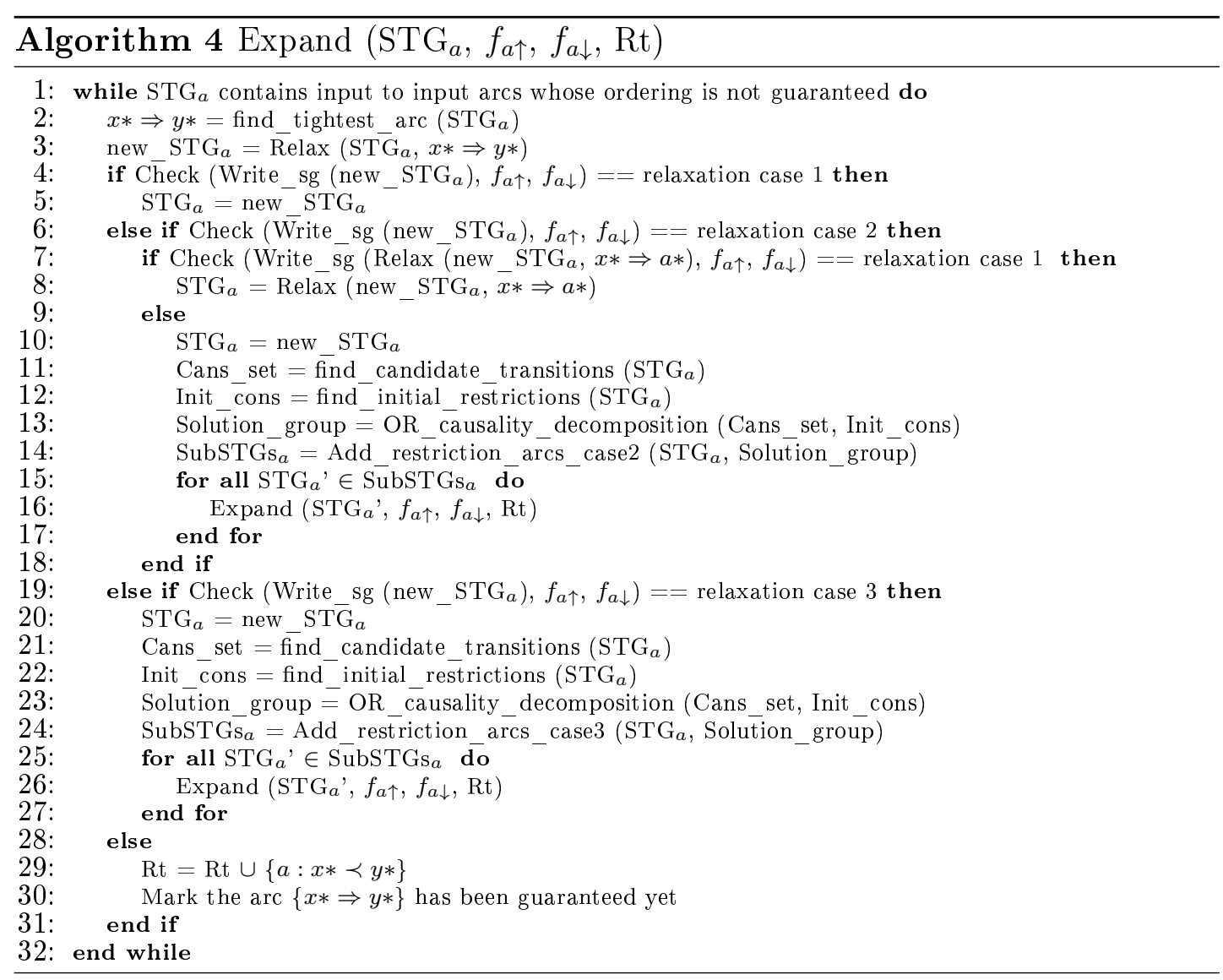

\subsubsection{Proof of correctness}

The starting point of the proposed method is an SI circuit and an STG describing its behavior. The SI circuit conforms to the STG, which means that the circuit is functionally correct and hazard-free. The relaxation operation only relaxes arcs between two transitions on the input signals of a gate, which is equivalent to changing two ordered input transitions into concurrent. This modification does 


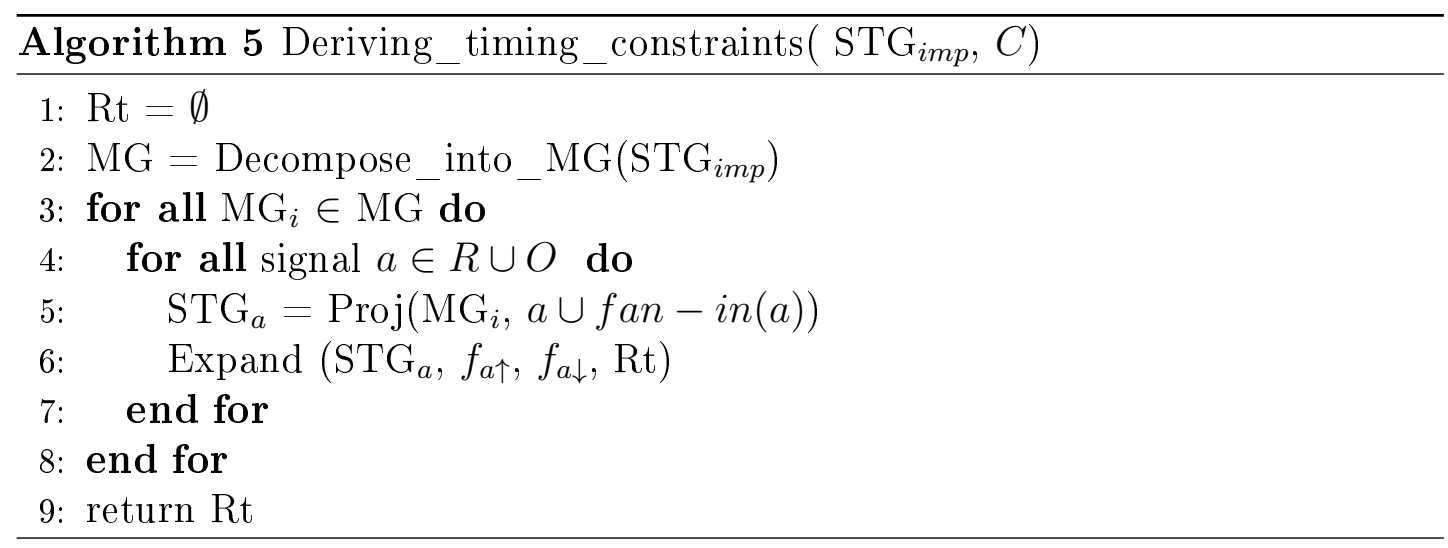

not change the interface protocol between a gate and its environment and thus could only introduce hazards but will not harm the functionality of the gate. Interface protocol will only be changed if the OR-causality relation happens, where the corresponding transition on output signal will be triggered by a different clause of the gate. However, this is a local effect, that the environment could only receive the output transition when it has sent the input transitions required by the gate in the initial STG. As will be analyzed in the next chapter, the subSTGs generated by the proposed OR-causality decomposition method cover all possible firing sequences when an OR-causality occurs. The relaxation operation will keep the liveness and consistency of the STG nor will the safeness be violated if the gate does not contain redundant literals. These three properties guarantee that when an arc in the STG is relaxed the resulting STG is still a valid representation of the behavior of the gate. Each time when an arc is relaxed, this relaxation will be accepted if it does not introduce hazards; or will be rejected if it does.

In section 5.3.2 we have proved that the relaxation operation does not destroy the liveness and consistency of the STG. Also, the safeness will not be affected if the circuit does not have redundant literals. This suggests that the whole process 
will eventually terminate. Indeed, when an arc is relaxed, more states could be reached by the resulting STG (if the relaxed arc is not a redundant one). For a live and safe STG with $n$ signals, there are at most $2^{n}$ reachable states. The whole process will eventually converge.

\subsection{Delay padding to fulfill timing constraints}

When the relaxation is done, all of the generated timing constraints could be changed into the pairwise delay constraints between a wire and a path by tracking back to the $\mathrm{STG}_{i m p}$ and looking up the Circuit $C$. The circuit will work correctly if these constraints are guaranteed. Some constraints could be considered to be "safe" (e.g. adversary path cross environment or a very long adversary path), some constraints could be guaranteed by layout. If there are very strong constraints (very short adversary path) and the technology variations are severe, all these strong constraints could be guaranteed by delay padding.

The padding technique will not be investigated in detail, because the number of strong constraints in an SI is usually not large. Therefore, finding a good padding positions in a circuit is not a difficult task when the timing constraints have been generated. A simple heuristic that could find a good padding positions in most cases is introduced below.

There are two kinds of padding positions: padding on a wire and padding on a gate. Figure 5.25 shows the possible padding positions (position 1-5) to guarantee the delay constraint that a wire from gate $g_{-} 1$ to $g_{-} 4$ should be faster than another path between these two gates. Padding on position 1, 3 or 5 
(padding on wire), only delays transitions on one branch of a gate; while padding on position 2 or 4 (padding on gate) will delay all branches of a gate output, which is equivalent to the increase of the delay of a gate. Padding on a gate could always fulfill one delay constraint without worsening other delay constraints, but might unnecessarily delay other branches in a fork; while padding on a wire has less performance penalty but may degrade another delay constraint if the wire that the delay padded on should be faster than another adversary path. A greedy padding policy could be used which tries to pad the delay on position 1 if the corresponding wire does not participate in another delay constraint. If it does, then the greedy algorithm tries to pad on position 3. In the worst case when all the wires in the adversary path are in some other delay constraints then pad on the position 2 could break this cyclic dependency.

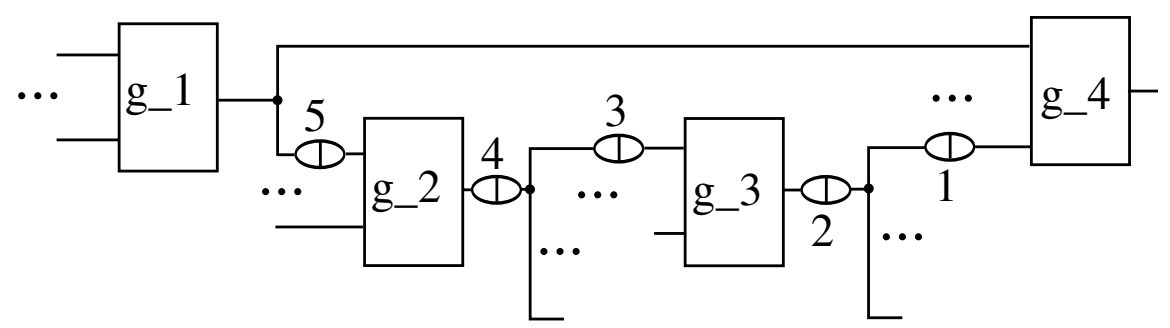

Figure 5.25: Padding positions -

Due to the padding rule described above it could be guaranteed that all of the delay constraints could be fulfilled (padding on the last gate could always fulfill this delay constraint without worsening another path, like the technique used in synthesis in [9]). Usually, a good padding method to get the minimum performance penalty is to try to pad delays on the wire near the destination gate of an adversary path such that this wire is not in the fast path of another delay 
constraint by looking up the delay constraint table(as was exemplified in Table 7.1 in Chapter 7).

\subsection{Summary}

This chapter introduces our method to generate a set of timing constraints for an SI circuit to work correctly when the isochronic fork timing assumption is relaxed into the intra-operator timing assumption. The method generates the local environments for all gates in the circuit and operates on these local environments to avoid exploring the reachability space of the entire circuit. In each local environment, the timing constraints implied by the isochronic fork will be relaxed one by one. Unnecessary timing constraints will be removed in the relaxation process. The computational complexity of the proposed method is polynomial to the number of signals in the circuit and the generated timing constraints could always be fulfilled. 


\section{Chapter 6}

\section{OR-causality Decomposition}

The relaxation case 2 and case 3 presented in the chapter 5 do not imply a glitch at a gate. They violate the timing conformance because the so-called OR-causality happens. This chapter analyzes the situation in which an OR-causality relation would appear in the local STG in relaxation case 2 and case 3 and proposes a method to decompose the STG into a set of subSTGs. The subSTGs are then treated as individual STGs and are processed one by one iteratively. A gate will work properly if it works properly in every subSTG.

\subsection{OR-causality}

The flow semantics of PNs, an event is enabled if and only if all of its input places have tokens, explicitly expresses the strong causality (AND-causality) between events. It is easy to describe the causality like this: events $a$ and $b$ must occur before the event $c$. However, the weak causality (OR-causality) like: event $c$ is 


\subsection{OR-causality}

enabled if any event of $a$ and $b$ occurs, is not easy to be represented in PNs. This kind of causality might appear in the local STG during the relaxation process. When two ordered transitions in the local STG are made to be concurrent by the relaxation operation, more than one clause could evaluate from false to true concurrently. The corresponding pull up/down function would become true and cause the gate to transit when any of its clause evaluates true. OR-causality relations between clauses cannot be expressed by a safe or free-choice PN. In order to describe the behavior of a gate using safe MGs where the proposed relaxation method could work, the local STG needs to be decomposed into a set of subSTGs when the OR-causality relations between transitions are encountered. The OR-causality in relaxation case 2 and case 3 will be analyzed in detail in this section and the decomposition method will be introduced in the next section.

\subsubsection{OR-causality in relaxation case 2}

OR-causality in relaxation case 2: Relaxation case 2 happens if and only if there is a transition $y *$ which is a prerequisite transition of $o *$ and the relaxation of an $\operatorname{arc} x * \Rightarrow y *$ makes $x *$ to be a prerequisite transition of $o *$, but $o *$ could also be caused by other transitions if $x *$ is delayed. Due to the relaxation rule, $x *$ must then be made concurrent with $o *$ because the transitions in the prerequisite set of $o *$ are those that must be necessary for the firing of $o *$. However, if the clause containing $x *$ is the last clause whose literals are all ordered with $o *$ then making $x *$ concurrent with $o *$ will cause $f_{o \uparrow}$ to become false in some states in $E R(o+)$ or $f_{o \downarrow}$ to become false in some states in $E R(o-)$. 


\subsection{OR-causality}

The Figure 6.1 shows an example that contains OR-causality in relaxation case 2. The pull up function $f_{o \uparrow}$ of the gate $o$ has three clauses, $x \cdot y, z \cdot k \cdot y$ and $m \cdot y \cdot n$. Initially, when the arc $k+\Rightarrow y+$ in the STG in diagram (b) is relaxed, $k+$ is placed to be a prerequisite transition of $o+$ as is shown in diagram (c). Apparently, $f_{o \uparrow}$ could also become true if clause $x \cdot y$ or $m \cdot n \cdot y$ becomes true. So, $k+$ needs to be modified to be concurrent with $o+$ by relaxing the arc $k+\Rightarrow o+$. The resulting STG shown in diagram (d) will be accepted because all the literals in clause $x \cdot y$ are still ordered with $o+$ which guarantees that $f_{o \uparrow}$ is true in each state in $E R(o+)$. However, when the arc $x+\Rightarrow y+$ in the STG in diagram (d) is relaxed, $x+$ should also be modified to be concurrent with $o+$. After that, the STG in diagram (f) could enter $E R(o+)$ after $m+$ and $y+$ fires but the pull up function of the gate $o, f_{o \uparrow}$, is still false.

The reason for this is that in the STG segment, any of the three clauses $x \cdot y$, $z \cdot k \cdot y$ and $m \cdot y \cdot n$ could cause $f_{o \uparrow}$ to become true, thus only the transition $y+$, which corresponds to their common literal $y$, is necessary for $o+$. All other transitions cannot be placed at the prerequisite transitions of $o+$. But it is required that all literals in at least one clause are ordered with $o+$ to guarantee that when the STG enters the $E R(o+)$ this clause could enable gate $o$ to transit to high. When any literal $(x+$ in this example) of the last clause that are ordered with $o+$ (the clause $x \cdot y$ in this example) is modified to be concurrent with $o+$, the STG could enter $E R(o+)$ without any clause becoming true.

When more than one clause could become true simultaneously and enable the gate to transit, a safe MG cannot describe this race behavior. In order to describe the behavior of a gate under OR-causality using MGs, the original local STG 


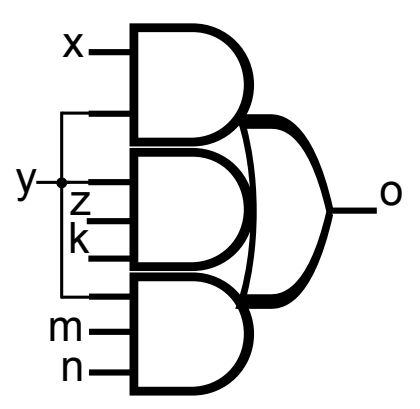

(a)

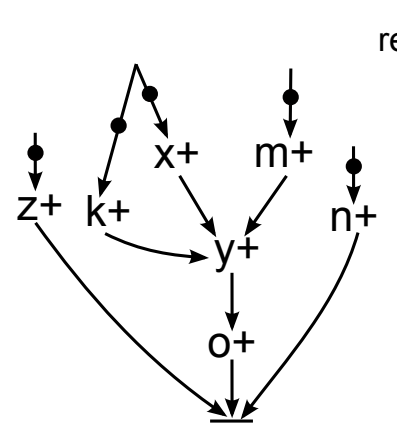

(b) relax $\mathrm{k}+->\mathrm{y}+$

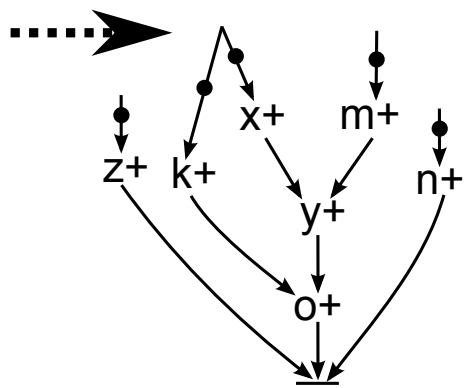

(c)

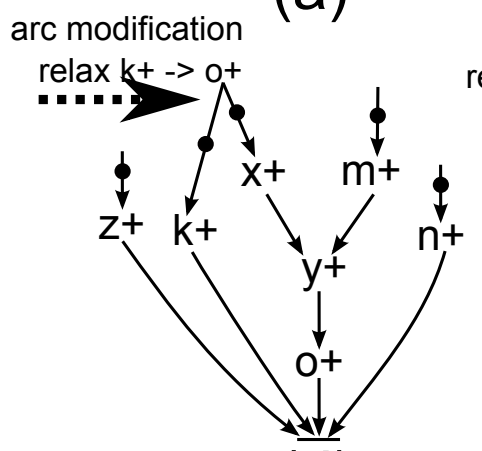

(d)

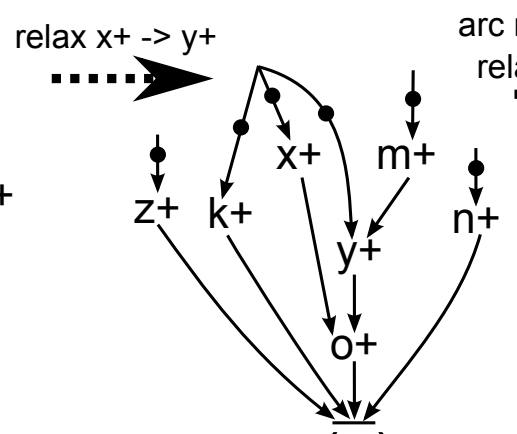

(e)

arc modification
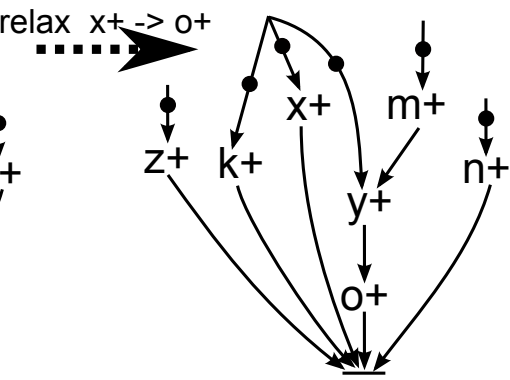

(f)

Figure 6.1: OR-causality in relaxation case 2. (a) the gate and (b)(f) relaxation steps - 


\subsection{OR-causality}

needs to be decomposed into a set of subSTGs. The OR-causality decomposition is done by adding concurrency restriction arcs between the transitions involved in the OR-causality relation, to guarantee that for each subSTG, one clause will always evaluate true before (or not later than if clauses have common literals) all other clauses, the transition on output signal is then unambiguously caused by that clause.

Candidate clauses are the clauses which have a possibility to win the race to cause the transition on the output signal. The candidate clauses for the ORcausality in relaxation case 2 could be derived by testing whether one clause could firstly evaluate true among all clauses in the "problematic" states.

Candidate clause for OR-causality in relaxation case 2 : In relaxation case 2, when the OR-causality happens, a clause $c$ in $f_{o \uparrow}$ (if $f_{o \uparrow}$ is false in some states in $E R(o+))$ is called a candidate clause if,

(1) In the SG corresponding to the STG before arc modification, there exist two states $s$ and $s^{\prime}$ in $Q R(o-), s \rightarrow s^{\prime}, f_{o \uparrow}(s)=$ false, $f_{o \uparrow}\left(s^{\prime}\right)=$ true and clause $c$ is true in $s^{\prime}$.

Or,

(2) Clause $c$ contains all prerequisite transitions of $o *$.

A clause $c$ in $f_{o \downarrow}$ (if $f_{o \downarrow}$ is false in some states in $E R(o-)$ ) is called a candidate clause if,

(1) In the SG corresponding to the STG before arc modification, there exist two states $s$ and $s^{\prime} \in Q R(o+), s \rightarrow s^{\prime}, f_{o \downarrow}(s)=$ false, $f_{o \downarrow}\left(s^{\prime}\right)=$ true and clause $c$ is true in $s^{\prime}$

Or, 
(2) Clause $c$ contains all prerequisite transitions of $o *$.

Not all transitions, which correspond to literals in a candidate clause, will be used for the OR-causality decomposition. Some transitions are guaranteed to occur before the OR-causality relation happens by the arcs in the current STG. The decomposition only involves those transitions whose occurrences could influence the order of clause evaluation.

Candidate transition for OR-causality decomposition: A transition $t *$ is called a candidate transition for the OR-causality decomposition if,

(1) there is a candidate clause $c, t \in c$ if $t *$ is $t+, \bar{t} \in c$ if $t *$ is $t-$ and $t *$ is concurrent with $o *$.

(2) $t *$ is $x *$, if this OR-causality is caused by relaxing $x * \Rightarrow y *$.

A candidate clause is a clause which could make $f_{o \uparrow}$ from false to true in $Q R(o-)$ or $f_{o \downarrow}$ from false to true in $Q R(o+)$ when the OR-causality happens. Candidate transitions are those transitions whose literals appear at a candidate clause and are concurrent with $o *$ or this transition is $x *$.

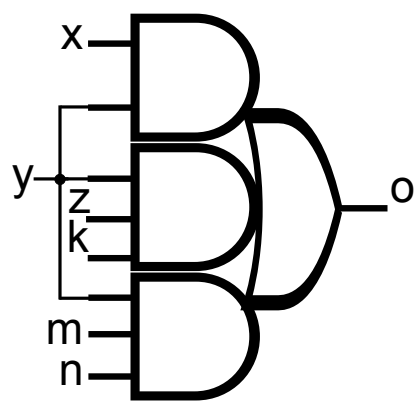

(a)

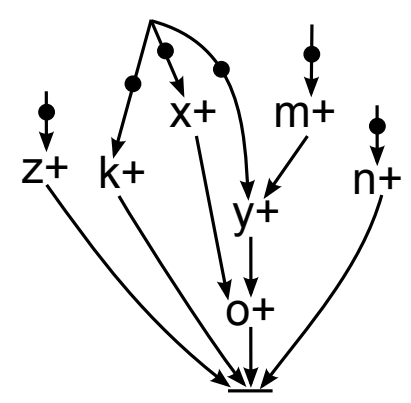

(b)

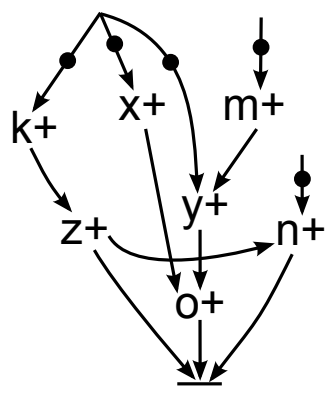

(c)

Figure 6.2: Candidate clause and transition for OR-causality in relaxation case 2. (a) the gate, (b) and (c) two different STG segments - 


\subsection{OR-causality}

Figure 6.2 shows a gate $o$ in diagram (a) together with two different STG segments in diagrams (b) and (c). In both STG segments, relaxation case 2 happens after relaxing $x+\Rightarrow y+$ and after modifying the $x+$ to be concurrent with $o+$, OR-causality happens. There are three candidate clauses $z \cdot k \cdot y, m \cdot n \cdot y$ and $x \cdot y$ in the STG segment in diagram (b), but only two candidate clauses $z \cdot k \cdot y$ and $x \cdot y$ in the STG segment in diagram (c). In diagram (b), when the transitions $m+, y+, z+$ and $k+$ fire the $f_{o \uparrow}$ turns from false to true; when the transitions $m+, y+$ and $n+$ fire the $f_{o \uparrow}$ turns from false to true. However, in diagram (c), there are no firing sequences by which the clause $m \cdot n \cdot y$ could make $f_{o \uparrow}$ turn from false to true, because when the clause $m \cdot n \cdot y$ becomes true the clause $z \cdot k \cdot y$ has already become true. The candidate transitions in diagram (b) are $z+, k+$ for the candidate clause $z \cdot k \cdot y(y+$ is not concurrent with $o+), n+$ for the candidate clause $m \cdot n \cdot y(m+$ and $y+$ are not concurrent with $o+)$ and $x+$ for the candidate clause $x \cdot y$. The candidate transitions in diagram (c) are $z+, k+$ for the candidate clause $z \cdot k \cdot y$ and $x+$ for the candidate clause $x \cdot y$.

\subsubsection{OR-causality in relaxation case 3}

OR-causality in relaxation case 3: Relaxation case 3 happens if and only if $x *$ is a prerequisite transition of $o *$ and the relaxation of an $\operatorname{arc} x * \Rightarrow y *$, which

makes $y *$ to be concurrent with $x *$, causes at least one clause containing the literal $y$ but not containing the literal $x$ in $f_{o \uparrow}$ to become true in $Q R(o-)$ or in $f_{o \downarrow}$ to become true in $Q R(o+)$.

The Figure 6.3 shows an example that contains OR-causality in relaxation 


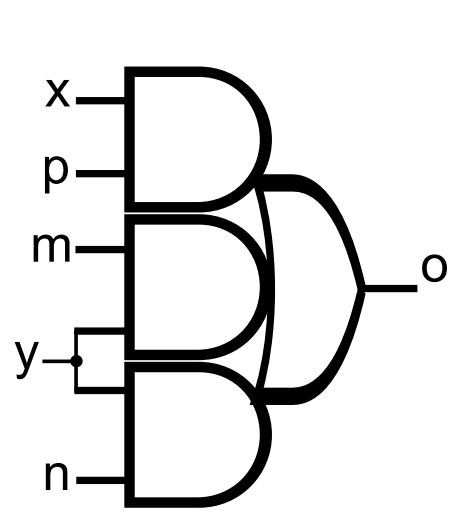

(a)

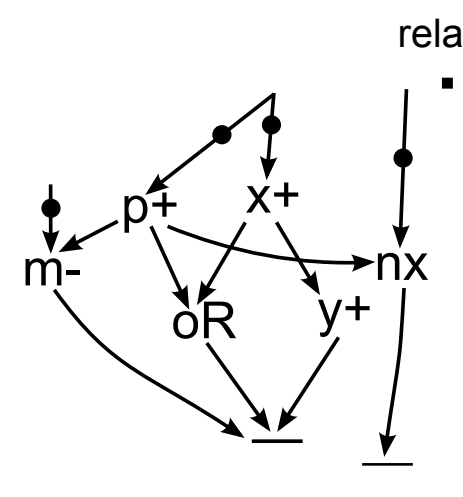

(b)

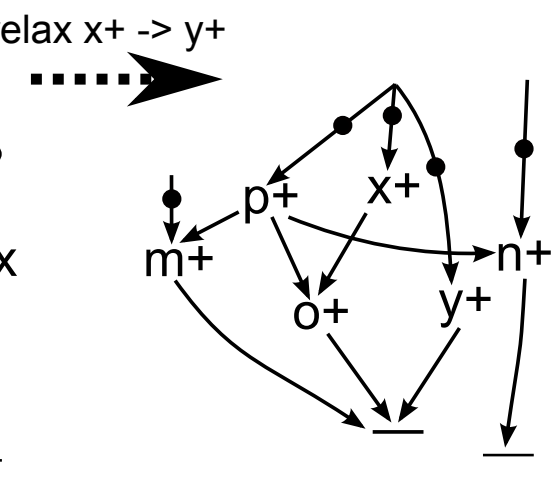

(c)

Figure 6.3: OR-causality in relaxation case 3. (a) the gate, (b) and (c) two different STG segments -

case 3. The pull up function $f_{o \uparrow}$ of the gate $o$ has three clauses, $p \cdot x, y \cdot m$ and $y \cdot n$. When the arc $x+\Rightarrow y+$ is relaxed, the pull up function $f_{o \uparrow}$ will become true if $m+$ or $n+$ arrives before $x+$, thus making the gate $o$ excited in $Q R(o-)$.

Before the arc $x+\Rightarrow y+$ is relaxed, the clause $p \cdot x$ is guaranteed to become true before clauses $y \cdot m$ and $y \cdot n$. So, $o+$ must be caused by the clause $p \cdot x$. When the arc $x+\Rightarrow y+$ is relaxed, these three clauses could become true concurrently and the $o+$ will be caused by the first one becoming true. This OR-causality still cannot be depicted by a single safe MG and the STG needs to be decomposed into a set of subSTGs.

Candidate clause for OR-causality in relaxation case 3 : In relaxation case 3 , when the OR-causality happens, a clause $c$ in $f_{o \uparrow}$ (if $f_{o \uparrow}$ is true in some states in $Q R(o-))$ is called a candidate clause if,

(1) In the SG corresponding to the STG, there exist two states $s$ and $s^{\prime}$ 


\subsection{OR-causality}

$\in Q R(o-), s \rightarrow s^{\prime}, f_{o \uparrow}(s)=$ false, $f_{o \uparrow}\left(s^{\prime}\right)=$ true and clause $c$ is true in $s^{\prime}$.

Or,

(2) The clause $c$ is the clause that contains all prerequisite transitions of $o *$.

A clause $c$ in $f_{o \downarrow}$ (if $f_{o \downarrow}$ is true in some states in $Q R(o+)$ ) is called a candidate clause if,

(1) In the SG corresponding to the STG, there exist two states $s$ and $s^{\prime}$ $\in Q R(o+), s \rightarrow s^{\prime}, f_{o \downarrow}(s)=$ false, $f_{o \downarrow}\left(s^{\prime}\right)=$ true and clause $c$ is true in $s^{\prime}$.

Or,

(2) The clause $c$ is the clause that contains all prerequisite transitions of $o *$.

Candidate transition for OR-causality decomposition: A transition $t *$ is called a candidate transition for OR-causality decomposition if,

(1) there is a candidate clause $c, t \in c$ if $t *$ is $t+, \bar{t} \in c$ if $t *$ is $t-$ and $t *$ is concurrent with $O *$.

(2) $t *$ is $x *$, if this OR-causality is caused by relaxing $x * \Rightarrow y *$.

Figure 6.4 shows a gate $o$ in diagram (a) together with two different STG segments in diagrams (b) and (c). In both STG segments, OR-causality happens. There are three candidate clauses $y \cdot m, y \cdot n$ and $p \cdot x$ in the STG segment in diagram (b), but only two candidate clauses $y \cdot n$ and $p \cdot x$ in the STG segment in diagram (c). In diagram (b), when the transitions $p+, m+$ and $y+$ fire the $f_{o \uparrow}$ turns from false to true; when the transitions $p+, n+$ and $y+$ fire the $f_{o \uparrow}$ turns from false to true. However, in diagram (c), there are no firing sequences by which the clause $y n$ could make $f_{o \uparrow}$ turn from false to true, because when the clause $y \cdot n$ becomes true the clause $y \cdot m$ has already become true. The candidate transitions in diagram (b) are $m+, y+$ for the candidate clause $y \cdot m, n+, y+$ for 
the candidate clause $y \cdot n$ and $x+$ for the candidate clause $p \cdot x$. The candidate transitions in diagram (c) are $m+, y+$ for the candidate clause $y \cdot m$ and $x+$ for the candidate clause $p \cdot x$.

Both OR-causality relations between clauses in relaxation cases 2 and 3 need the STG to be decomposed into a set of subSTGs, and the technique that achieves this will be introduced in the next section.

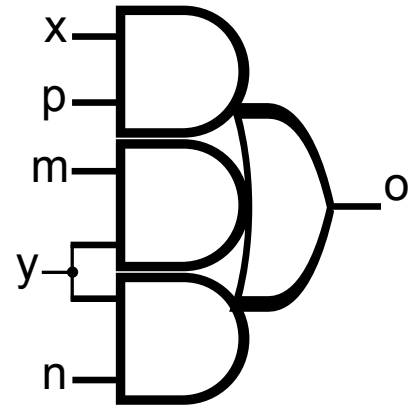

(a)

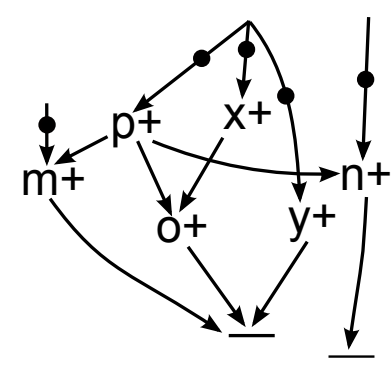

(b)

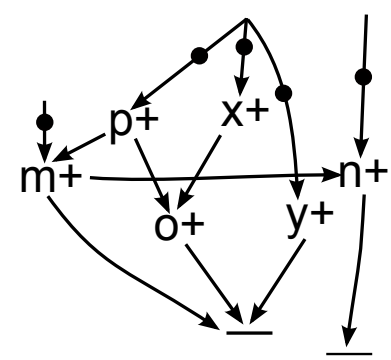

(c)

Figure 6.4: Candidate clause and transition for OR-causality in relaxation case 3. (a) the gate, (b) and (c) two different STG segments -

\subsection{Decomposition of OR-causality}

OR-causality during the relaxation is the situation when the isochronic fork timing assumption is relaxed, more than one clause in a pull-up or pull-down function of a gate could (depending on which of them become true first) cause a transition at the gate's output. This race relation cannot be depicted by a safe MG. In order to describe the behavior of a gate using MG, the corresponding local STG needs to be decomposed into a set of subSTGs where each subSTG does not 


\subsection{Decomposition of OR-causality}

contain OR-causality relations and the union of reachable states of all subSTGs should contain all the states that could be reached by the original STG when OR-causality happens.

The diagrams (a) and (b) in Figure 6.5 show a gate $o$ and its local STG segment that were introduced before in Figure 6.2. The STG segment in diagram (b) contains OR-causality relations where any of the three clauses $x \cdot y, z \cdot k \cdot y$ and $m \cdot n \cdot y$ could trigger a rising transition at the gate $o$ output when it becomes true and there are no constraints on which of these three clauses will become true first. Diagrams (c) to (g) are the decomposed subSTG segments of the STG segment in diagram (b). In each subSTG, the OR-causality relation is eliminated by adding order-restriction arcs (arcs marked with a '\#' symbol). The order-restriction arcs are added between candidate transitions in an ORcausality, which restrict the orderings of candidate transitions in different clauses to guarantee that one candidate clause will always become true before all others, the output transition is then clarified to be caused by that clause. For example, the diagrams (c) and (d) depict the subSTGs where the output transition $o+$ is caused by the clause $x \cdot y$, diagram (e) depicts the subSTG where $o+$ is caused by clause $z \cdot k \cdot y$ and diagram (f) and (g) depict the subSTGs where $o+$ is caused by clause $m \cdot n \cdot y$. The OR-causality decomposition only restricts the ordering between the candidate transitions, so, the transition $m+$ in the diagram will not be considered in the decomposition but it will be considered when the arc $m+\Rightarrow y+$ is relaxed and the corresponding STG entering OR-causality again in the following subSTG relaxation. The union of the states reached by diagram (c) to $(\mathrm{g})$ includes all the states that the gate $o$ could exhibit in diagram (b). 


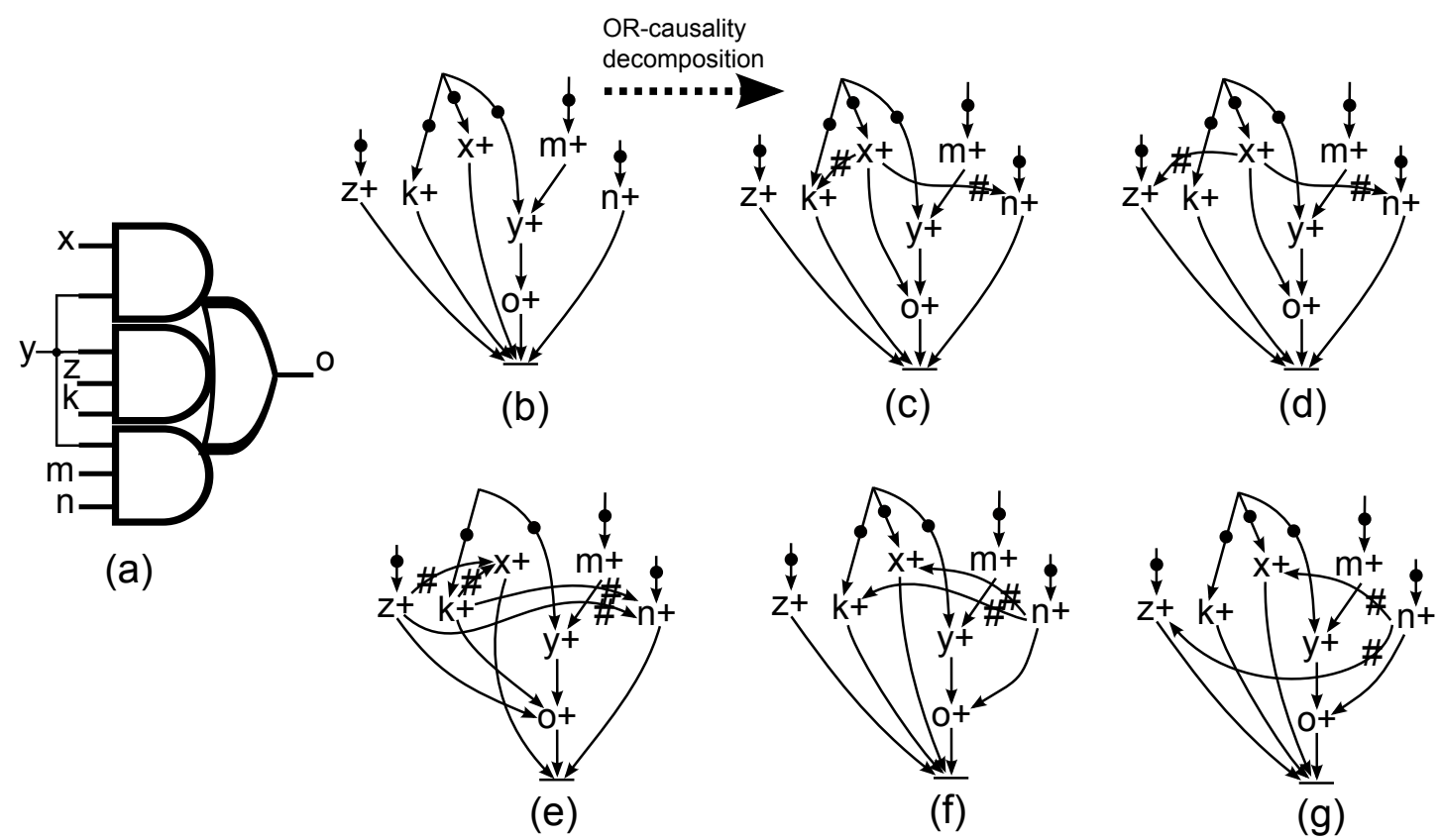

Figure 6.5: OR-causality decomposition example. (a) the gate, (b) STG segment before decomposition and (c)-(g)resulting subSTG segments - 


\subsection{Decomposition of OR-causality}

An order-restriction arc behaves like a normal arc in $\mathrm{PN}$ semantics except that it will not be relaxed (the ordering of the two events connected by a orderrestriction arc is considered to be fixed) and it will not be checked for redundancy, which means even if an order-restriction arc is redundant it will not be eliminated. The elimination of an order-restriction arc might cause STG entering OR-causality relation again in the future relaxation and cause the additional unnecessary OR-causality decomposition. Keep the redundant order-restriction arcs in an STG will not cause any trouble, because they will not be relaxed any more.

The technique to decompose an STG which contains an OR-causality will be demonstrated on a set of examples, from simple to complex.

The decomposition of an STG containing OR-causality is a technique to add order-restriction arcs to concurrent candidate transitions between different clauses to make sure that one clause will become true before other clauses. The subSTGs should include all the states that could be reached by the gate under this ORcausality and the decomposition that contains fewer subSTGs is preferred (each subSTG needs to be analyzed one by one, fewer subSTGs implies less computation).

A set of concurrency restriction arcs are generated for each subSTG during the OR-causality decomposition. The subSTG is then derived by adding these arcs into the original STG and modifying some causalities when needed. For example, the set of concurrency restriction arcs for the subSTG in diagram (c) in Figure 6.5 are $\{x+\prec k+; x+\prec n+\}$. The solution of an OR-causality decomposition is a group of sets of concurrency restriction arcs, one set for each subSTG. 
The solution for the OR-causality in Figure 6.5 is:

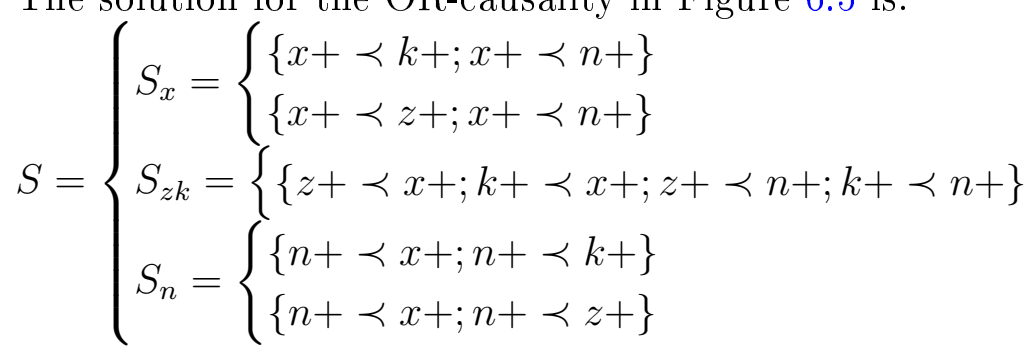

Firstly, consider the simplest situation, where an OR-causality relation only involves two clauses $c_{a}$ and $c_{b}$. The clause $c_{a}$ will become true when all the candidate transitions in set $\mathcal{A}$ fire and the clause $c_{b}$ will become true when all transitions in set $\mathcal{B}$ fire. The task is to generate a set of subSTGs such that in each subSTG the clause $c_{a}$ evaluates true before the clause $c_{b}$, and the set of states in the SGs of all subSTGs includes all the states in which the clause $c_{a}$ will evaluate true before the clause $c_{b}$.

For example, suppose the clause $c_{a}$ becomes true when all the candidate transitions in set $\mathcal{A}=\{a+, b+, c+\}$ fire and clause $c_{b}$ becomes true when all the candidate transitions in set $\mathcal{B}=\{d+, e+, f+\}$ fire. Initially, there are no constraints about the firing order relation among these transitions. There are $P_{6}^{6}=720$ different firing sequences. When a restriction $a+\prec d+$ is added, it excludes all firing sequences that $d+$ fires before $a+$. The question is how to find a group of restriction sets such that in each constraint set, there are only pairwise order constraints between two transitions (like $a+\prec d+$ ). The order constraints in a constraint set work together to limit the firing sequences this constraint set expresses. The union of the firing sequences of all constraint sets contains all sequences in which all transitions in set $\mathcal{A}$ fire before at least one transition in set $\mathcal{B}$ and contains no sequences in which all transitions in set $\mathcal{B}$ fire before at least 


\subsection{Decomposition of OR-causality}

one transition in set $\mathcal{A}$. Among all of the groups which fulfill the requirement above, the one which contains the fewest number of sets is preferred.

For example, in the group $\left\{\begin{array}{l}\{a+\prec d+; b+\prec e+\} \\ \{a+\prec d+; b+\prec e+; c+\prec d+\}\end{array}\right.$, there are two restriction sets $\{a+\prec d+; b+\prec e+\}$ and $\{a+\prec d+; b+\prec e+; c+\prec d+\}$. This group is not a valid solution for the problem: Firstly, the restriction set $\{a+\prec$ $d+; b+\prec e+\}$ includes a firing sequence $a+\rightarrow d+\rightarrow b+\rightarrow e+\rightarrow f+\rightarrow c+$ where all transitions in $\mathcal{B}$ fire before the transition $c+$ in $\mathcal{A}$. Besides, the firing sequence $e+\rightarrow b+\rightarrow a+\rightarrow c+\rightarrow d+\rightarrow f+$, which should be included, is not included in any restriction set.

A closer look at problem description reveals that, each transition in $\mathcal{A}$ should fire before at least one transition in $\mathcal{B}$, this requires that each transition $t * \in \mathcal{A}$ to have a constraint pair $t * \prec t^{\prime} *$ where $t^{\prime} * \in \mathcal{B}^{1}$. Otherwise, there exists a firing sequence where all transitions in $\mathcal{B}$ fire before $t *$ and thus violates the requirement. Meanwhile, each transition $t * \in \mathcal{A}$ needs at most one constraint pair $t * \prec t^{\prime} *$ where $t^{\prime} * \in \mathcal{B}$. Additional order constraints on $t *$ only exclude some required firing sequences and push the solution into sub-optimal.

The technique to get the required solution group will be analyzed step by step, from the simplest to the most general case.

\footnotetext{
${ }^{1}$ This is under the circumstances that $t *$ does not transitively fire before any transition in $\mathcal{B}$, this situation will be analyzed in detail later
} 


\subsection{Decomposition of OR-causality}

\subsubsection{Generating the solution group}

This subsection will introduce the method to derive the solution group step by step.

Case (1): There are only two clauses involved in an OR-causality whose candidate transition sets are $\mathcal{A}$ and $\mathcal{B}$. Sets $\mathcal{A}$ and $\mathcal{B}$ do not have any common transition and do not have initial ordering restrictions between transitions. The task is to generate a group of restriction sets for the relation $\mathcal{A} \prec \mathcal{B}$, where $\mathcal{A} \prec \mathcal{B}$ denotes all the firing sequences that all transitions in $\mathcal{A}$ must fire before at least one transition in $\mathcal{B}$ :

In all valid firing sequences, the last transition must be a transition in set $\mathcal{B}$, and the task is to generate a group of restriction sets to include these and only these sequences. When the sets $\mathcal{A}$ and $\mathcal{B}$ do not have any common transition and do not have initial ordering restrictions between transitions, the method to generate the solution group is quite straightforward: generate a restriction set for each transition $t^{\prime} *$ in set $\mathcal{B}$ and in each restriction set generate order constraint pairs to make all transitions in $\mathcal{A}$ fire before $t^{\prime} *$. For example when the set $\mathcal{A}=\{a+, b+, c+\}$ and $\mathcal{B}=\{d+, e+, f+\}$. The solution group $S_{\mathcal{A} \prec \mathcal{B}}=$ $\left\{\begin{array}{l}\{a+\prec d+; b+\prec d+; c+\prec d+\} \\ \{a+\prec e+; b+\prec e+; c+\prec e+\} \\ \{a+\prec f+; b+\prec f+; c+\prec f+\}\end{array} \quad\right.$ is valid (and quite likely to be optimal). The constraint set $\{a+\prec d+; b+\prec d+; c+\prec d+\}$ includes (but not only includes) all the firing sequences ending with transition $d+$ and so do $\{a+\prec e+; b+\prec$ $e+; c+\prec e+\}$ and $\{a+\prec f+; b+\prec f+; c+\prec f+\}$ for transition $e+$ and $f+$. In all three constraint sets, every transition in $\mathcal{A}$ is forced to precede one transition in $\mathcal{B}$, so all the firing sequences included by three restriction sets are valid. The 


\subsection{Decomposition of OR-causality}

solution group contains $|\mathcal{B}|$ restriction sets, where $|\mathcal{B}|$ denotes the cardinality of the set $\mathcal{B}$.

The case (1) solves the simplest case where there are only two candidate clauses, which do not have any common candidate transitions and all candidate transitions are concurrent. The case (2) will allow two candidate clauses to have common candidate transitions, based on case (1).

Case (2): Sets $\mathcal{A}$ and $\mathcal{B}$ do not have initial ordering restrictions between transitions, but might have common transitions:

When $\mathcal{A}$ and $\mathcal{B}$ have common transitions, for example $\mathcal{A}=\{a+, b+, c+\}$ and $\mathcal{B}=\{a+, d+, e+, f+\}$. Transition $a+$ is a candidate transition, which is necessary for both two clauses to become true. In this case, $a+$ does not need to have an order restriction to precede any other transition in $\mathcal{B}$ (or it could be considered as the transition $a+$ in $\mathcal{A}$ always precedes itself in $\mathcal{B}$ ). All transitions that are both in set $\mathcal{A}$ and $\mathcal{B}$ will be eliminated from set $\mathcal{A}$ and then, the new set $\mathcal{A}^{\prime}$ and $\mathcal{B}$ do not have any common transitions and do not have initial ordering restrictions between transitions.

After deleting the common transitions, $\mathcal{A}^{\prime}$ and $\mathcal{B}$ fulfill the conditions in case (1) and the constraint group could be derived using the method shown in case

(1). The constraint group for $\mathcal{A}=\{a+, b+, c+\}$ and $\mathcal{B}=\{a+, d+, e+, f+\}$ for the relation $\mathcal{A} \prec \mathcal{B}$ is $S_{\mathcal{A} \prec \mathcal{B}}=\left\{\begin{array}{l}\{b+\prec a+; c+\prec a+\} \\ \{b+\prec d+; c+\prec d+\} \\ \{b+\prec e+; c+\prec e+\} \\ \{b+\prec f+; c+\prec f+\}\end{array}\right.$.

There could be initial ordering restrictions between transitions in $\mathcal{A}$ and $\mathcal{B}$ when all candidate transitions are not fully concurrent in the STG. Except for 


\subsection{Decomposition of OR-causality}

fulfilling all requirements for $\mathcal{A} \prec \mathcal{B}$, the solution group should not involve any firing sequences that contradict the initial ordering restrictions. This means that if there is a transitive relation that $t * \prec t^{\prime} *$, where $t *, t^{\prime} * \in \mathcal{A} \cup \mathcal{B}$; the solution group should not contain any firing sequence where $t^{\prime} *$ appears before $t *$. The case (3) allows two candidate clauses to have initial ordering restrictions between candidate transitions.

Case (3): Sets $\mathcal{A}$ and $\mathcal{B}$ could have initial ordering restrictions between transitions and could contain common transitions:

This is the most general case between two transition sets $\mathcal{A}$ and $\mathcal{B}$. There might be some pre-set ordering relations between transitions in set $\mathcal{A}$ and $\mathcal{B}$. For example, when $\mathcal{A}=\{a+, b+, c+, g+, h+\}, \mathcal{B}=\{a+, d+, e+, f+\}$ with the initial orderings $\{c+\prec d+; f+\prec c+; e+\prec b+; e+\prec g+\}$. The initial orderings are from ordering relations between candidate transitions in the STG specification. As in case (2), the common transitions of $\mathcal{A}$ and $\mathcal{B}$ will be removed from $\mathcal{A}$ first. The set $\mathcal{A}$ after eliminating the common transitions is $\mathcal{A}^{\prime}=\{b+, c+, g+, h+\}$. Meanwhile, for any transition $t * \in \mathcal{A}$ if there is a transitivity relation $t * \prec$ $t_{1} *, t_{1} * \prec \ldots \prec t_{m} *, t_{m} * \prec t_{n} *$ where $t_{1} *, \ldots, t_{m} * \in \mathcal{A} \cup \mathcal{B}$ and $t_{n} * \in \mathcal{B}$, it implies that $t *$ is already guaranteed to precede the transition $t_{n} * \in \mathcal{B}$ and $t *$ does not need any additional ordering restrictions. All transitions in $\mathcal{A}$, which are already guaranteed to precede one transition in $\mathcal{B}$, will be eliminated from $A$. The set $\mathcal{A}$ after eliminating the common transitions in $\mathcal{B}$ and the transitions which are already guaranteed to precede one transition in $\mathcal{B}$ is $\mathcal{A}^{\prime \prime}=\{b+, g+, h+\}$.

The question is then changed into how to find the required restriction group between $\mathcal{A}^{\prime \prime}$ and $\mathcal{B}$ such that $\mathcal{A}^{\prime \prime}$ and $\mathcal{B}$ do not have any common alphabets and 


\subsection{Decomposition of OR-causality}

there are no transitions in $\mathcal{A}^{\prime \prime}$ which transitively precede a transition in $\mathcal{B}$ but there could be some transitions in $\mathcal{B}$ which transitively precede a transition in $\mathcal{A}^{\prime \prime}$.

Any valid firing sequence for the relation $\mathcal{A}^{\prime \prime} \prec \mathcal{B}$ must be ended by a transition in $\mathcal{B}$. When a transition $t^{\prime} * \in \mathcal{B}$ transitively precedes a transition in $\mathcal{A}^{\prime \prime}$ in the initial ordering restrictions, transition $t^{\prime} *$ cannot be the last transition in any valid firing sequence.

From the previous analysis it could be seen that, when solving the solution group for the relation $\mathcal{A} \prec \mathcal{B}$ in case (2), one constraint set will be generated for each transition $t *$ in $\mathcal{B}$ to include all firing sequences ending with $t *$. When a transition $t^{\prime} * \in \mathcal{B}$ in case (3) cannot be the last transition in any valid firing sequence, no constraint set should be generated for this transition. So, the transition $t^{\prime} * \in \mathcal{B}$ should be deleted from the set $\mathcal{B}$. The set $\mathcal{B}$ after deleting those transitions that transitively precede a transition in $\mathcal{A}^{\prime \prime}$ is denoted by $\mathcal{B}^{\prime}$.

After deleting all the transitions in $\mathcal{A}^{\prime}$, which transitively precede a transition in $\mathcal{B}$ and the transitions in $\mathcal{B}$ which transitively precede a transition in $\mathcal{A}^{\prime}$ in the initial ordering restrictions. $\mathcal{A}^{\prime \prime}$ and $\mathcal{B}^{\prime}$ fulfill the conditions in case (1). The constraint group for $\mathcal{A}=\{a+, b+, c+, g+, h+\}, \mathcal{B}=\{a+, d+, e+, f+\}$ with the initial orderings $\{c+\prec d+; f+\prec c+; e+\prec b+; e+\prec g+\}$ is $\mathcal{A} \prec \mathcal{B}$ is $S_{\mathcal{A} \prec \mathcal{B}}=\left\{\begin{array}{l}\{b+\prec a+; c+\prec a+; g+\prec a+; h+\prec a+\} \\ \{b+\prec d+; c+\prec d+; g+\prec d+; h+\prec d+\}\end{array}\right.$.

To sum up, in order to find a solution group of the relation $\mathcal{A} \prec \mathcal{B}$ subject to a set of initial ordering restrictions, the common transitions of $\mathcal{A}$ and $\mathcal{B}$ and the transitions in $\mathcal{A}$ which transitively precede a transition in $\mathcal{B}$, are removed from $\mathcal{A}$ first. Then the transitions in $\mathcal{B}$ which transitively precede a transition in $\mathcal{A}$, are 
removed from $\mathcal{B}$. After this, a restriction set where all transitions of $\mathcal{A}$ precede a transition $t *$ will be generated for each transition $t * \in \mathcal{B}$. The algorithm for solving the relation $\mathcal{A} \prec \mathcal{B}$ subject to the initial ordering restrictions Init_cons is presented in Algorithm 6.

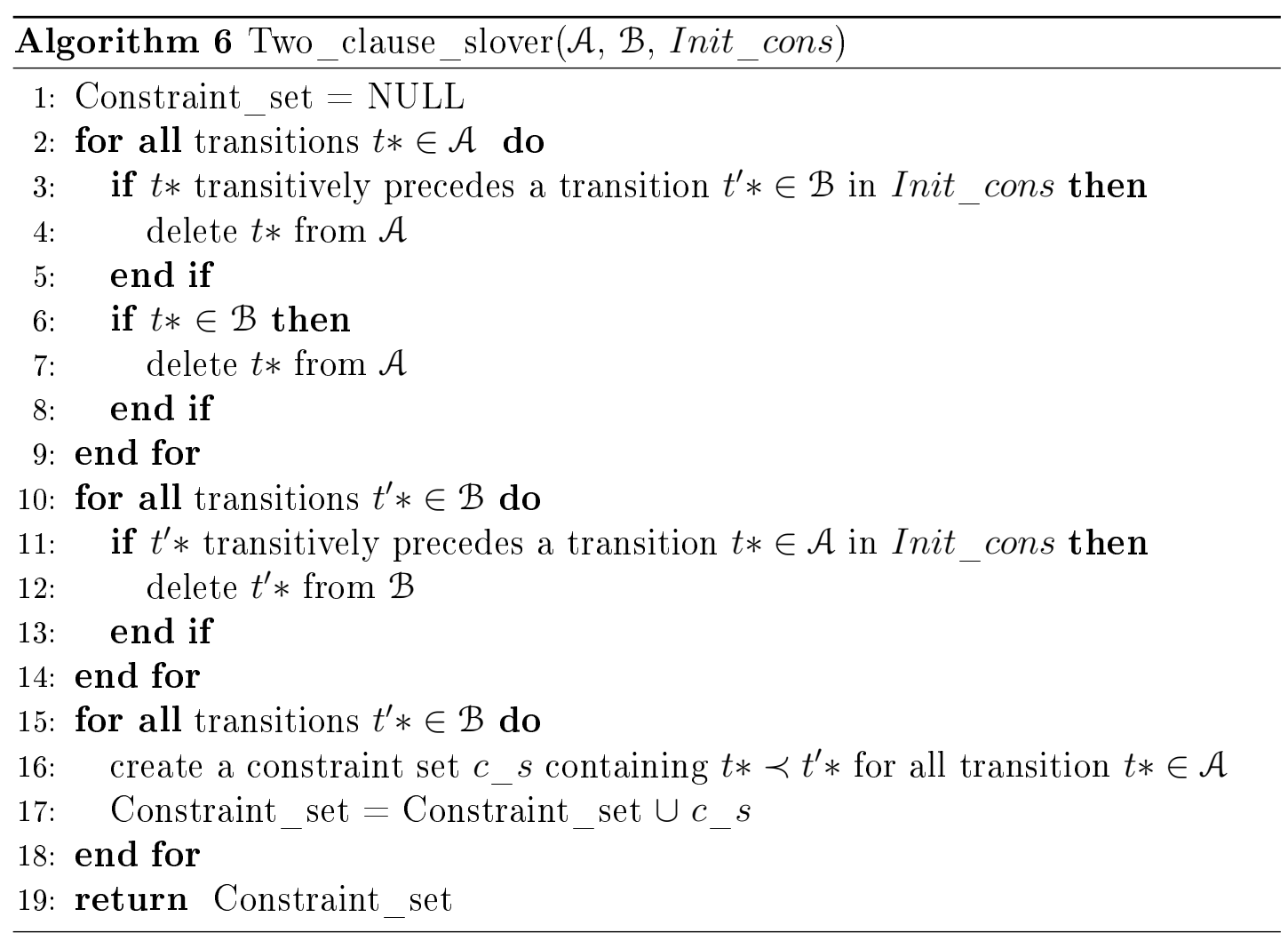

\subsubsection{Decomposition according to the solution group}

When OR-causality relation involves more than two candidate clauses, $c_{1}, c_{2}, \ldots, c_{n}$, the timing restrictions for a given clause $c_{1}$ to be evaluated true before all other clauses are solved in two steps. Firstly, derive the solution groups for $c_{1}$ to be evaluated true before each other clause separately, and then picks up a restriction set in each group and merge (union) them together to form a restriction set 


\subsection{Decomposition of OR-causality}

in the final solution group. The final solution group should include all possible combinations of restriction sets in each solution group.

For example, in the Figure 6.5 , the solution for the clause $m \cdot n \cdot y$ to be

evaluated true before the clause $x \cdot y$ is $S_{m n y \prec x y}=\{\{n+\prec x+\} \quad$ and to be evaluated true before the clause $z \cdot k \cdot y$ the restriction group is $S_{m n y \prec z k y}=$ $\left\{\begin{array}{l}\{n+\prec z+\} \\ \{n+\prec k+\}\end{array}\right.$. So, the solution for the clause $m \cdot n \cdot y$ to be firstly evaluated true among all clauses is $S_{m n y}=\left\{\begin{array}{l}\{n+\prec x+; n+\prec z+\} \\ \{n+\prec x+; n+\prec k+\}\end{array}\right.$.

There might exist common restriction sets between certain groups. For example, if the solution group for the relation $\mathcal{A} \prec \mathcal{B}$ is $S_{\mathcal{A} \prec \mathcal{B}}=\left\{\begin{array}{l}\{a+\prec c+; b+\prec c+\} \\ \{a+\prec d+; b+\prec d+\}\end{array}\right.$ and for $\mathcal{A} \prec \mathcal{C}$ is $S_{\mathcal{A} \prec e}=\left\{\begin{array}{l}\{a+\prec c+; b+\prec c+\} \\ \{a+\prec e+; b+\prec e+\}\end{array}\right.$. When the restriction set $\{a+\prec c+; b+\prec c+\}$ is picked from the solution group $S_{\mathcal{A} \prec \mathcal{B}}$, there is no need to pick any restriction set from the second group $S_{\mathcal{A} \prec e}$. So, when it is the turn to pick a restriction set from one group, whether any restriction set in that group has been included will be checked first. If any restriction set have been included (which is true if two groups have common restriction sets), this group will be ignored in this turn. Moveover, when two restriction sets have common restriction orderings, the repeated restriction orderings will be removed automatically by the union operation.

The algorithm for calculating the timing constraints for a clause, whose candidate transition set is $\mathcal{A}$, to be firstly evaluated true among all candidate clauses is presented in Algorithm 8. Algorithm 8 calls the Algorithm 7 to recursively solve all combinations of restriction sets (res_set) in each solution group(sub_sets).

The decomposition of an OR-causality, is to generate the timing restriction 

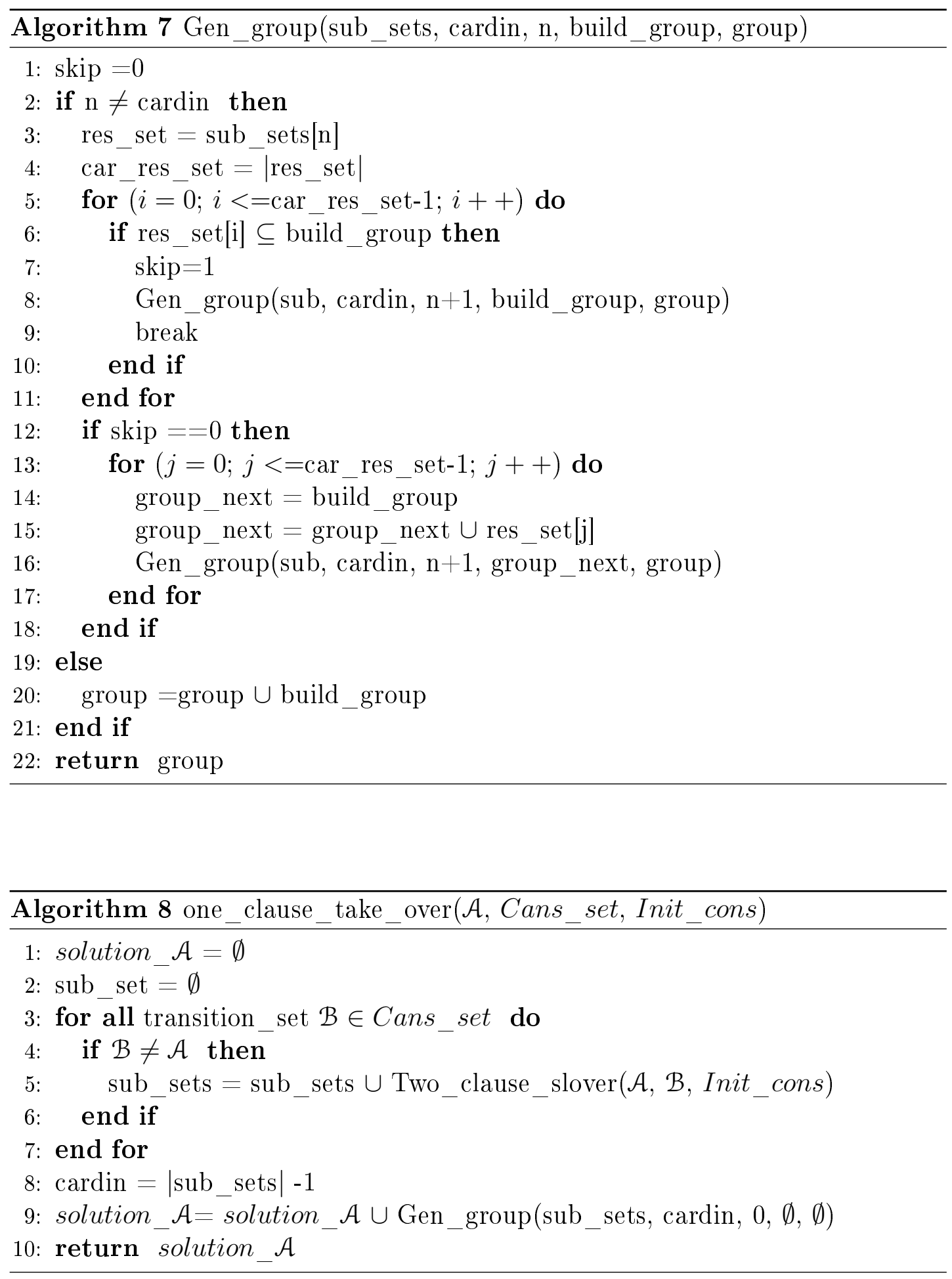


\subsection{Decomposition of OR-causality}

groups for each candidate clause, where each restriction set represents the added ordering restriction arcs in one subSTG. The top level algorithm for the decomposition of an OR-causality relation involving a set of candidate transition sets Cans_set with the initial ordering restriction set Init_cons is presented in Algorithm 9.

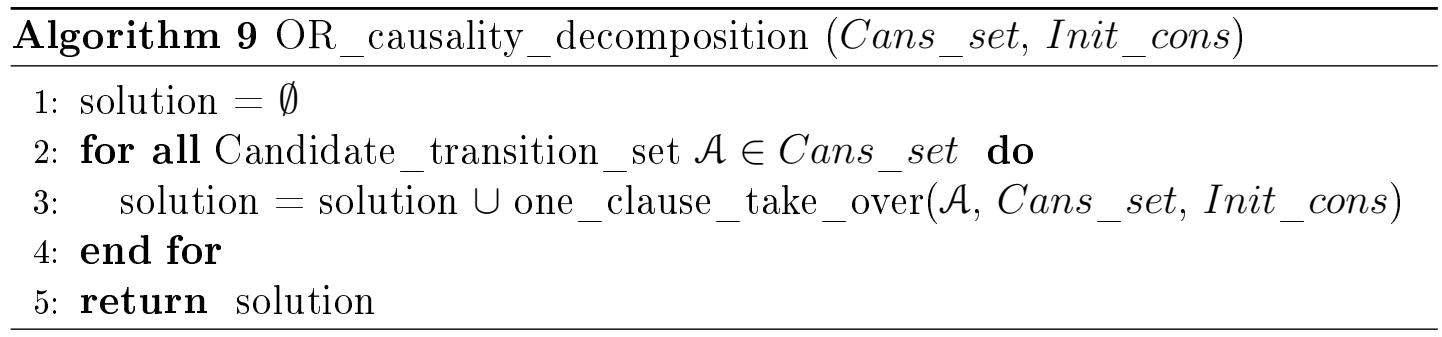

When the solution group for one OR-causality is derived, the STG will be decomposed into a set of subSTGs according to the restriction sets in the group. One subSTG will be generated for each restriction set and an ordering restriction arc will be inserted for each restriction pair in each restriction set.

For OR-causality relation in case 2, when one candidate clause takes responsibility for causing the output transition, arcs will be added from all candidate transitions in that clause to the output transition to indicate that these candidate transitions are the prerequisite transitions for the output transition. One ordering restriction arc (marked with \# symbol) will be added for one restriction pair in each restriction set.

Figure 6.6 shows a gate $o$ and its STG segment. This STG segment has an OR-causality relation whose solution set is 


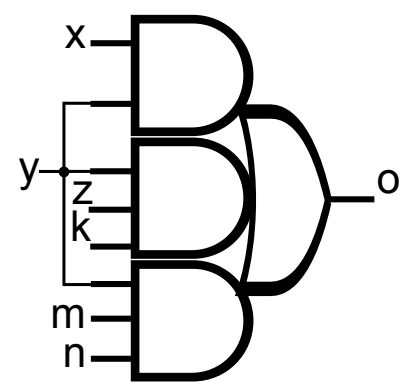

(a)

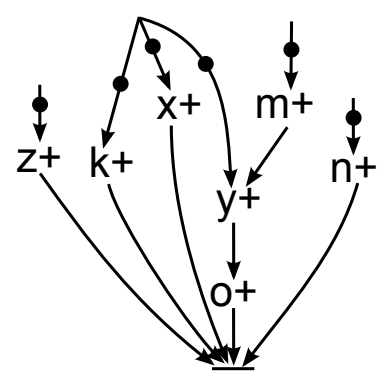

(b)

Figure 6.6: An OR-causality relation in case 2. (a) the gate and (b) its local STG segment -

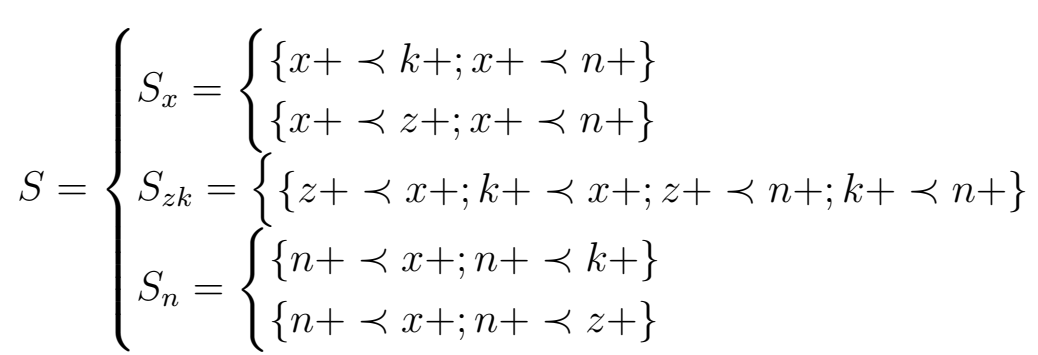

The decomposition results for the OR-causality relation in Figure 6.6 (after eliminating the redundant arcs) is presented in Figure 6.7. Diagram (a) in Figure 6.7 shows the subSTG corresponds to the restriction set $\{x+\prec k+; x+\prec$ $n+\}$. Diagram (b) shows the subSTG corresponds to the restriction set $\{x+\prec$ $z+; x+\prec n+\}$. Diagram (c) shows the subSTG corresponds to the restriction set $\{z+\prec x+; k+\prec x+; z+\prec n+; k+\prec n+\}$. Diagram (d) shows the subSTG corresponds to the restriction set $\{n+\prec x+; n+\prec k+\}$. Diagram (e) shows the subSTG corresponds to the restriction set $\{n+\prec x+; n+\prec z+\}$.

For OR-causality relation in case 3 , when one candidate clause $c$ takes charge of causing the output transition, arcs will be added from all candidate transitions in this clause to the output transition to indicate that these candidate transitions are the prerequisite transitions for the output transition. For all transitions $t *$, 


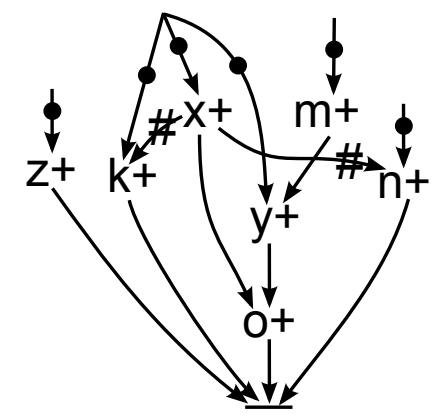

(a)

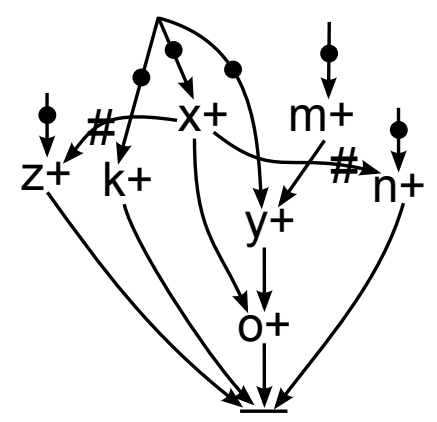

(b)

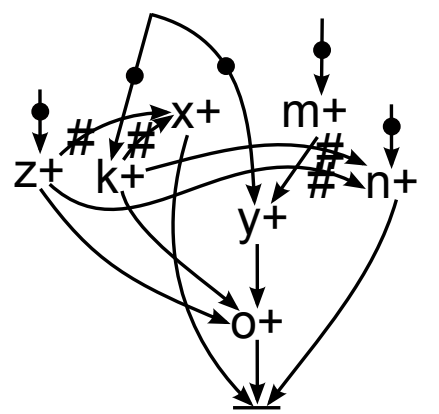

(c)

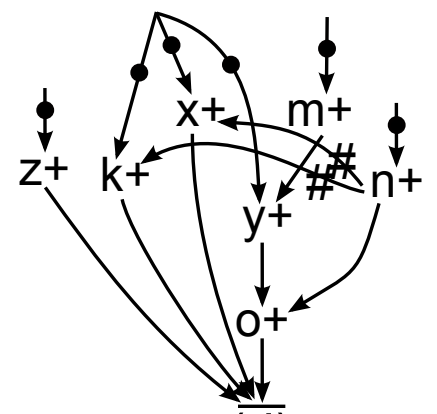

(d)

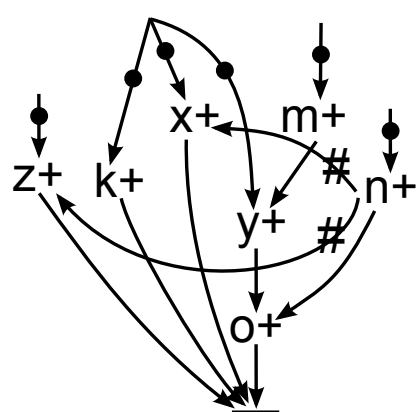

(e)

Figure 6.7: Decomposition results for the OR-causality relation in Figure 6.6. (a)-(e) resulting subSTG segments - 
which are prerequisite transitions for the output transition in the original STG, if $t$ (if $t *$ is $t+$ ) or $\bar{t}$ (if $t *$ is $t-$ ) is not a literal of clause $c$, the arc $t * \Rightarrow o *$ will be relaxed. This is because unlike case 2 , where all the prerequisite transitions of $o *$ are still prerequisite transitions after decomposition, if $t$ or $\bar{t}$ is not a literal of clause $c, t *$ will not be the prerequisite transition any more and $t *$ should become concurrent with $o *$. One ordering restriction arc (marked with \# symbol) will be added for one restriction pair in each restriction set.

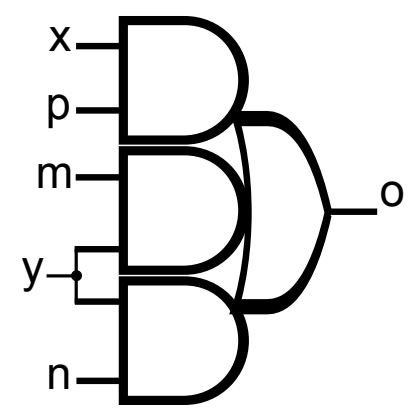

(a)

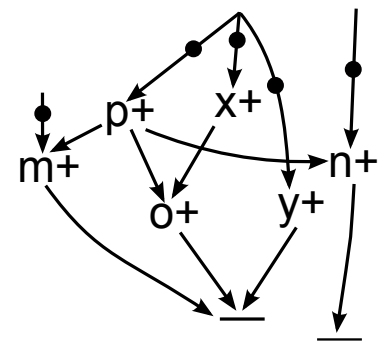

(b)

Figure 6.8: An OR-causality relation in case 3. (a) the gate and (b) its local STG segment -

Figure 6.8 shows a gate $o$ and its STG segment. This STG segment meets an OR-causality relation whose solution set is

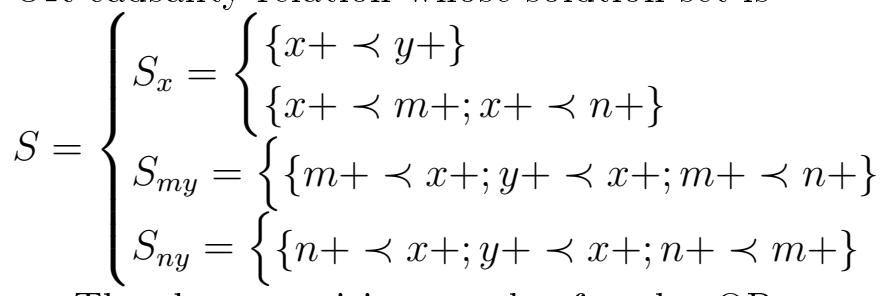

The decomposition results for the OR-causality relation in Figure 6.8 (after eliminating the redundant arcs) is presented in Figure 6.9. Diagram (a) in Figure 6.9 shows the subSTG corresponds to the restriction set $\{x+\prec y+\}$. Dia- 
gram (b) shows the subSTG corresponds to the restriction set $\{x+\prec m+; x+\prec$ $n+\}$. Diagram (c) shows the subSTG corresponds to the restriction set $\{m+\prec$ $x+; y+\prec x+; m+\prec n+\}$. Diagram (d) shows the subSTG corresponds to the restriction set $\{n+\prec x+; y+\prec x+; n+\prec m+\}$.

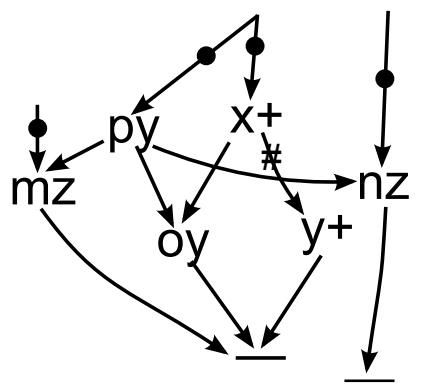

(a)

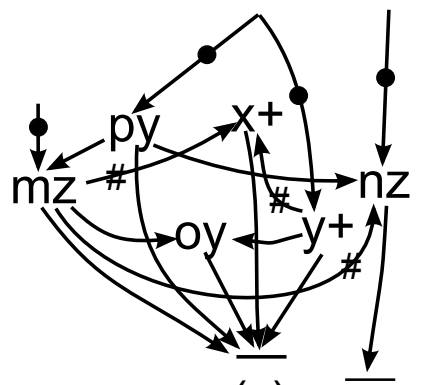

(c)

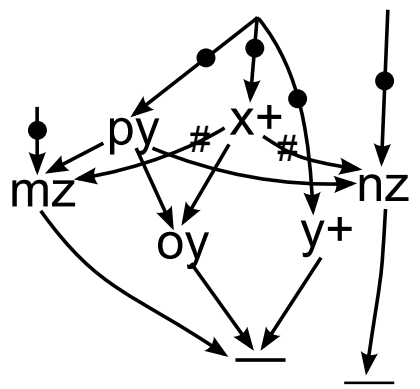

(b)

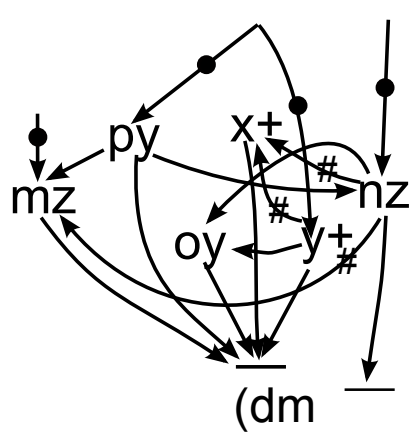

Figure 6.9: Decomposition results for the OR-causality relation in Figure 6.8. (a)-(d) resulting subSTG segments -

\subsection{Summary}

This chapter analyzes the OR-causality relation in the relaxation case 2 or case 3 in detail and introduces the technique to decompose an STG into a set of subSTGs when an OR-causality occurs. In each subSTG, one pull up/down function of a 
gate is guaranteed to be enabled by one specified clause. There are no ORcausality relations in the subSTGs. Each subSTG is then processed in the flow described in chapter 5 and a gate will work properly if it works properly in every subSTG. 


\section{Chapter 7}

\section{Results}

This chapter presents the results of the theory proposed in Chapter 5. In the first section, one example is used to demonstrate entire flow of the proposed method in detail and then the benchmark results for certain application examples are presented.

\subsection{Design example}

The inputs of the proposed technique are one SI circuit together with its implementation STG. As an example, the block diagram and the STG specification of a 2-cycle FIFO controller (chu150) is presented in Figure 7.1. The specification is synthesized and then decomposed into simple gates using the tool petrify. The resulting implementation STG and the circuit diagram are presented in Figure 7.2. This implementation STG is an MG, so, it does not need to be decomposed anymore. The local STG for each internal and output signal is then derived and 


\subsection{Design example}

processed independently. Here, the gate_Ai is chosen as an example.

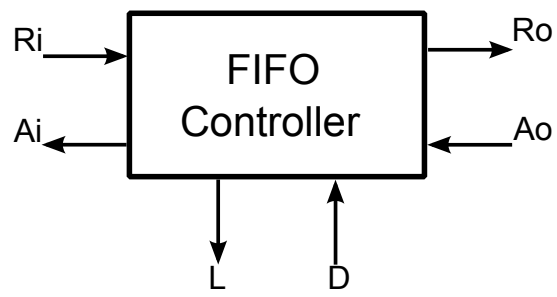

(a)

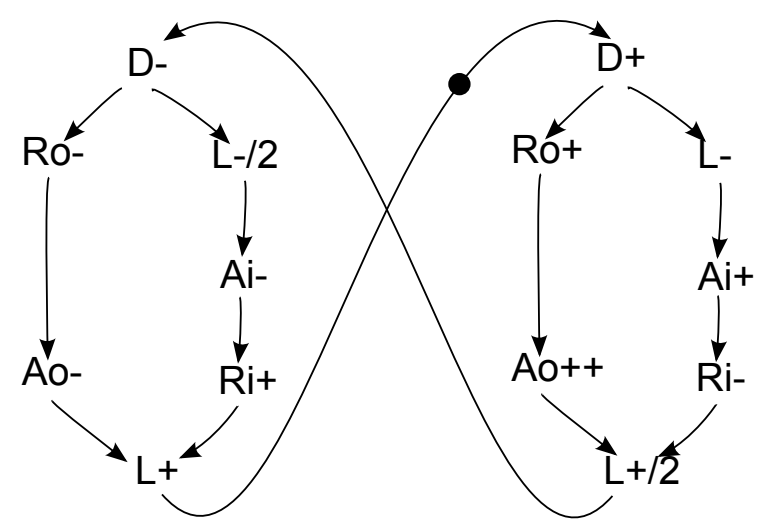

(b)

Figure 7.1: The block diagram and STG specification of FIFO -

The diagrams (a) and (b) in Figure 7.3 present the circuit diagram of gate_0 and its local STG. There are four timing ordering arcs $(L+\Rightarrow D+, D+\Rightarrow L-$, $L+/ 2 \Rightarrow D-$ and $D-\Rightarrow L-/ 2)$ in the local STG that rely on the isochronic fork timing assumption. The $\operatorname{arc} L+\Rightarrow D+$ is chosen to be relaxed first. The resulting STG is shown in diagram (c). The relaxation case 4 happens in the resulting STG, which suggests that glitches will appear if the transition $D+$ reaches gate_0 before $L+$. One timing constraint $L+\prec D+($ the arc marked with a \& symbol in diagram (d)) is added to the constraint set. Then in diagram (d), the arc $L+/ 2 \Rightarrow D-$ is chosen to be relaxed. In the resulting STG in diagram (e), the relaxation case 3 happens. The STG in diagram (e) is then decomposed into two subSTGs in diagrams (f) and (j) to solve the OR-causality (order-restriction arcs are marked with a \# symbol). The subSTGs in diagrams (f) and (j) are then analyzed individually. The relaxation process in each subSTG iterates until all ordering relations are guaranteed. The final subSTG are presented in diagrams 

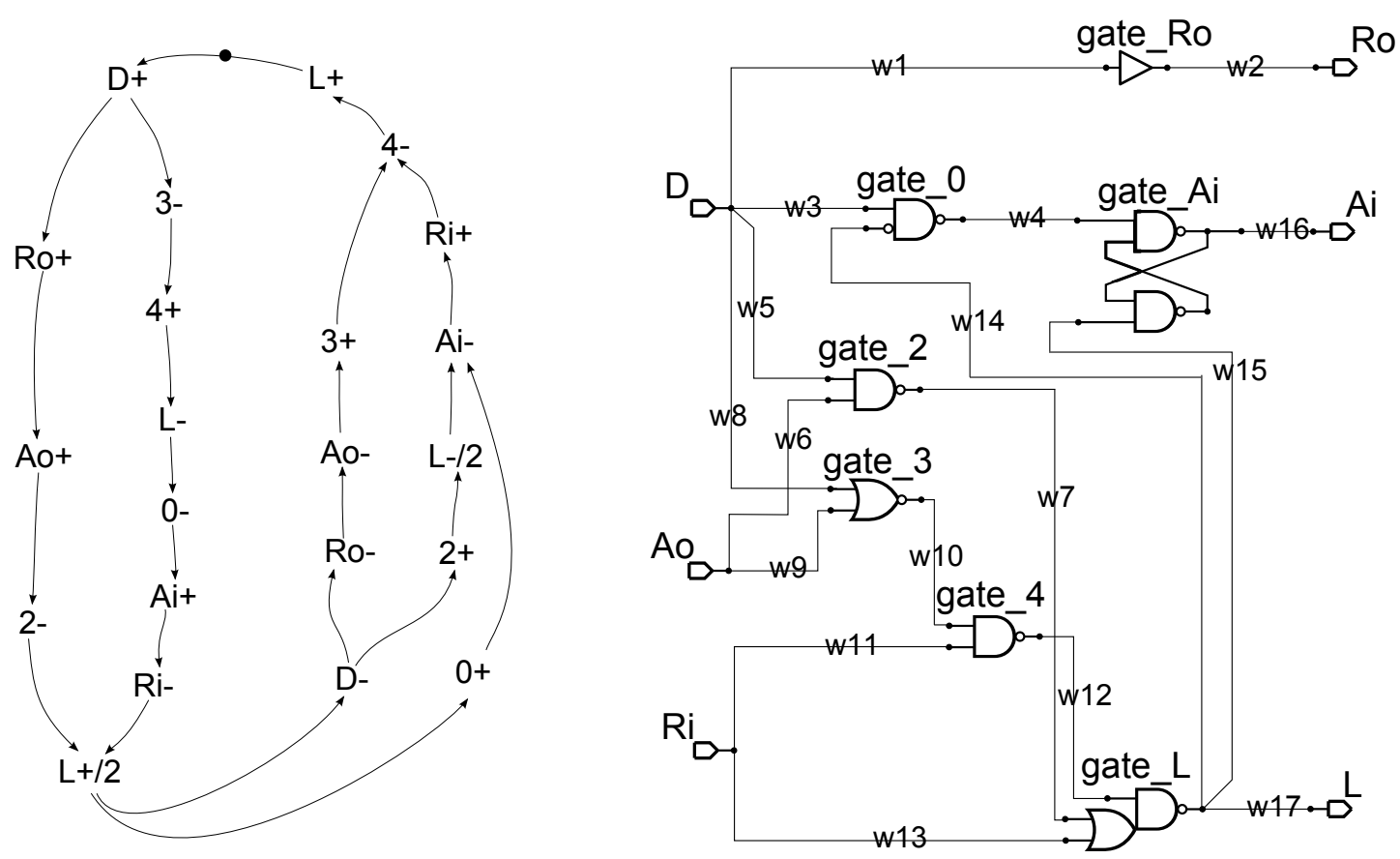

Figure 7.2: The implementation STG and circuit diagram of FIFO -

(i) and (k). One could see that there are two timing constraints $(L+\prec D+$ and $D-\prec L-/ 2$ ) in the final subSTGs compared with four in the original local STG, which indicates that the unnecessary timing orderings $D+\Rightarrow L-$ and $L+/ 2 \Rightarrow D-$ are excluded during the relaxation process.

A timing constraint could then be changed into a delay constraint between a wire and its adversary path by looking up the circuit and the entire STG shown in Figure 7.2. The timing constraint $D-\prec L-/ 2$ for gate_0 implies that the transition $D$ - from the environment propagating to gate_0 along the wire $w 3$ should be faster than propagating along the adversary path wire $w 5$, gate_2, $w 7$, gate_L and $w 14$.

Each internal and output signal in this circuit are processed individually, and 


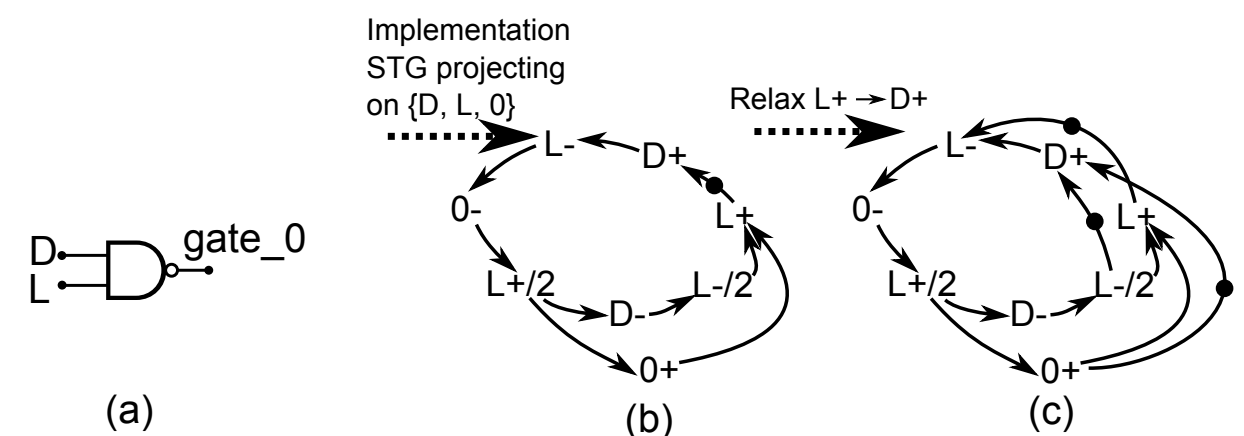

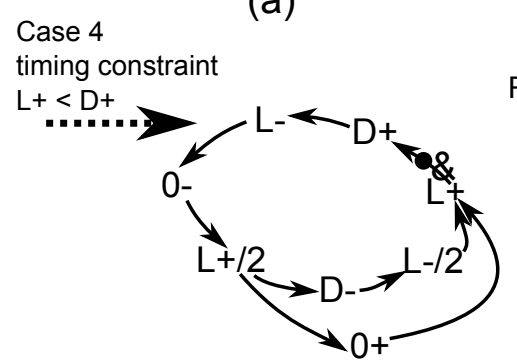

(d)

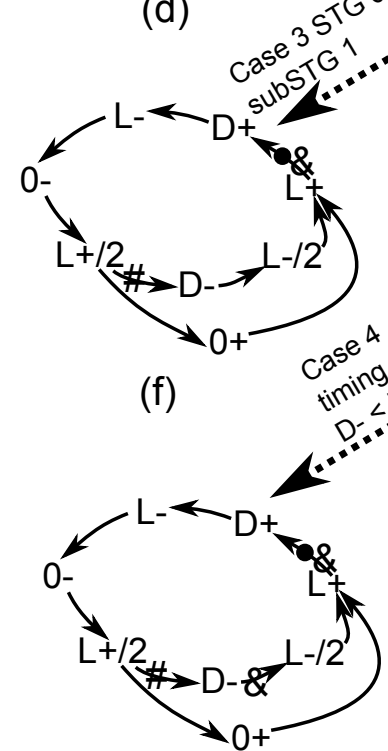

(h) (b)

(c)

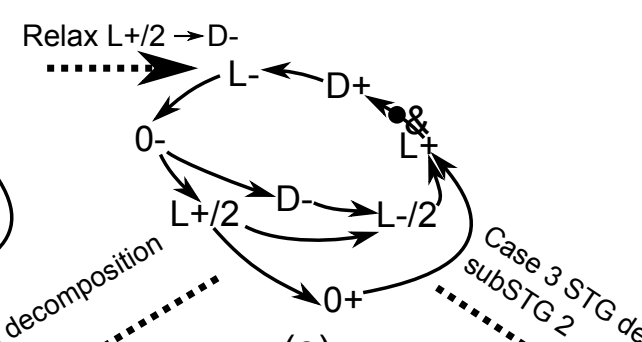

(e)

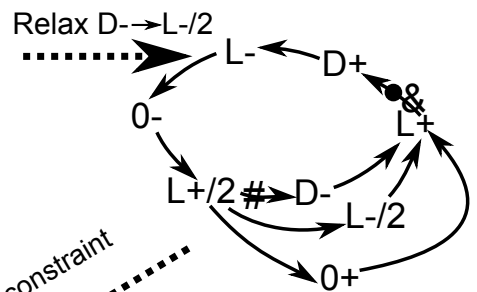

(g)

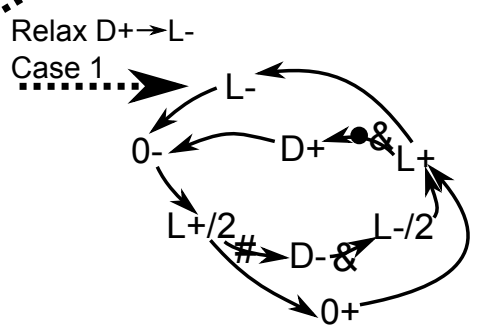

(i)

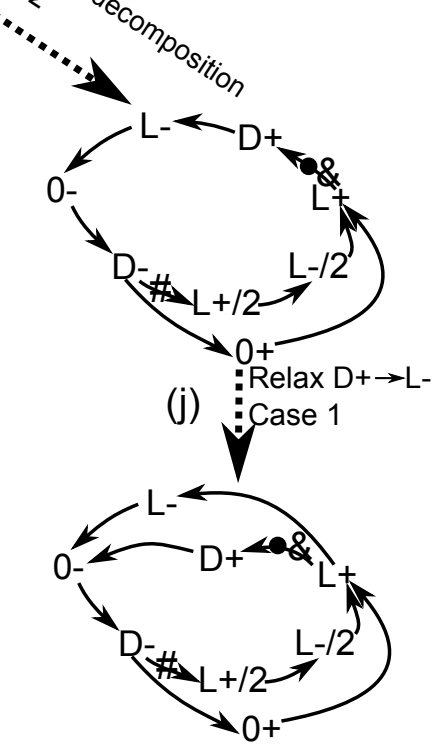

(k)

Figure 7.3: The STG relaxation procedure of the gate 0 - 


\subsection{Design example}

the delay constraints for this circuit to work correctly are shown in Table 7.1. Most constraints in Table 7.1 are quite loose, which could be considered to have been fulfilled already. When some constraints are considered strong, delays need to be padded to fulfill these constraints.

In this example, the adversary paths, whose levels are deeper than five (adversary path involves more than two gates), or which pass through the environment (the delay for the response from the environment is usually larger than a wire delay in the circuit), are considered to be fulfilled already. There are two constraints left, $w 15+<w 14+$,gate_ $0+, w 4+$ and $w 3-<w 5-$,gate_ $2+, w 7+$, gate_L-, $w 14-$. These two constraints could be guaranteed by padding delays on wire $w 4$ and $w 14$.

A closer look at Table 7.1 reveals that each constraint only involves unidirectional transitions on the adversary paths. This suggests that less performance penalty would be introduced if the delays padded on adversary paths only delay the required unidirectional transition. This could be achieved by using the current-starved delay[65][66]. Two examples of the current-starved delay are presented in Figure 7.4. The current-starved delay uses a control voltage $V$ to control the charge/discharge current to control the delay magnitude of falling/rising transitions. The delay presented in diagram (a) in Figure 7.4 could delay the rising transition for a given time but has little effect on the falling transition; while the current-starved delay in (b) could delay the falling transition for a given time but has little effects on the rising transition. 
Table 7.1: List of timing constraints

\begin{tabular}{|c|c|c|}
\hline wire & $\leq$ & adversary path \\
\hline $\mathrm{w} 15+$ & & w14+, gate_0+, w4+ \\
\hline $\mathrm{w} 14+$ & & $\mathrm{w} 3+, \mathrm{ENV}, \mathrm{w} 17+$ \\
\hline w3- & & w5-, gate_2+, w7+, gate_L-, w14- \\
\hline w6- & & w9-, gate_3+, w10+, gate_4-, w12-,gate_L+, w17+,ENV, w5+ \\
\hline w5- & & $\mathrm{w} 1+$, gate_Ro $+, \mathrm{w} 2+, \mathrm{ENV}, \mathrm{w} 6+$ \\
\hline $\mathrm{w} 8+$ & & 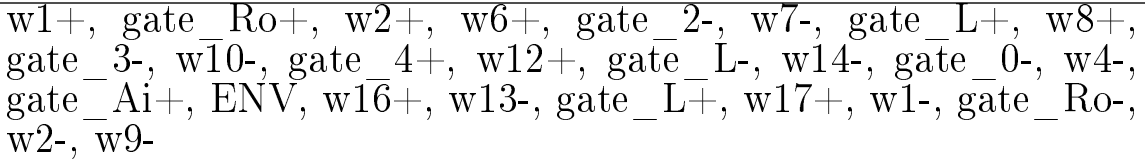 \\
\hline $\mathrm{w} 9+$ & & w6+, gate_2-, w7-, gate_L+, w17+, ENV, w8- \\
\hline $\mathrm{w} 13+$ & & $\begin{array}{l}\text { w11+, gate_4-, w12-, gate_L }+ \text {, w17+, ENV, w1+, Ro }+, \text { w2 }+ \text {, } \\
\text { ENV, w6 }+ \text {, gate_2-, w7- }\end{array}$ \\
\hline w11- & & $\begin{array}{l}\text { w13-, gate_L+, w17+, ENV, w1-, Ro-, w2-, w9-, gate_3+, } \\
\text { w10+ }\end{array}$ \\
\hline
\end{tabular}

\subsection{Simulation and Analysis}

The FIFO circuit is put through SPICE simulation using ASU Predictive Technology Model bulk CMOS model library from 90nm to 32nm [67]. The theoretical error rates due to the failure of the isochronic fork as the process shrinks and the performance penalty due to the padding are tested. When calculating the error rate, only the glitches caused by the wire delays are considered. Failures caused by the variation of the threshold are not considered. The error rate of the circuit is pessimistically calculated when any gate in the FIFO circuit glitches. As the length of local interconnection decreased as process shrinks, the relative wire length is used to compare the error rates between different processes. The wire length is changed into units of gate pitches and the interconnection distribution function of the wire length in a circuit is calculated using the formula in [68]: 


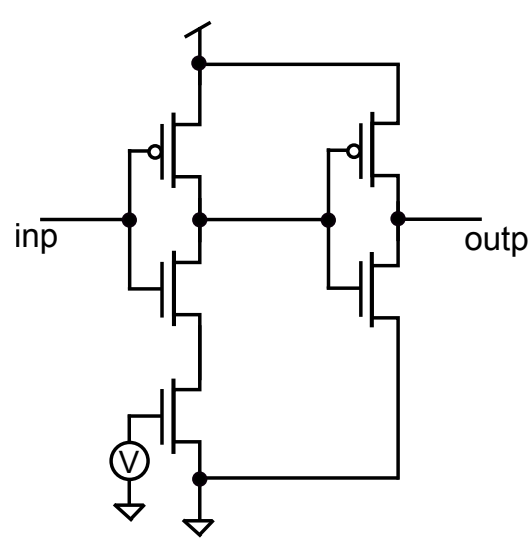

(a)

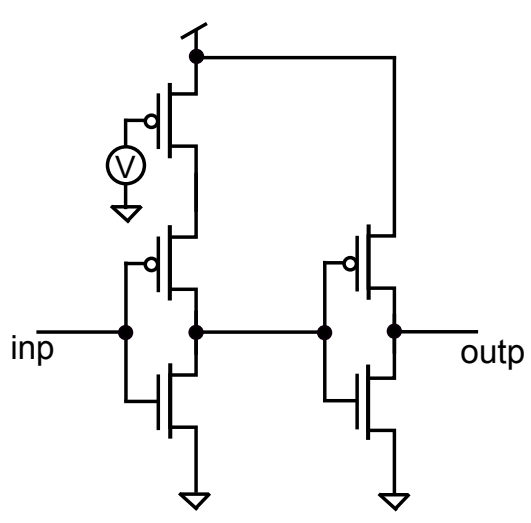

(b)

Figure 7.4: The current starved delays. (a) controlled delay for rising transition and (b) controlled delay for falling transition -

for $1 \leq l \leq \sqrt{N}$ :

$$
i(l)=\frac{\alpha k}{2} \Gamma\left(\frac{l^{3}}{3}-2 \sqrt{N} l^{2}+2 N l\right) l^{(2 p-4)}
$$

for $\sqrt{N} \leq l<\sqrt{2 N}$ :

$$
i(l)=\frac{\alpha k}{6} \Gamma(2 \sqrt{N}-l)^{3} l^{(2 p-4)}
$$

where

$$
\Gamma=\frac{2 N\left(1-N^{p-1}\right)}{-N^{p} \frac{1+2 p-2^{2 p-1}}{p(2 p-1)(p-1)(2 p-3)}-\frac{1}{6 p}+\frac{2 \sqrt{N}}{2 p-1}-\frac{N}{p-1}}
$$

Where $\mathrm{N}$ is the number of gates and the experience constant is set to $k=3, p=0.85$ and $\alpha=\frac{2}{3}$ respectively. The error rate for each gate is conservatively calculated as:

$$
E R=\int_{\text {error_length }}^{2 \sqrt{N}} i(l) d l \cdot\left(\int_{0}^{\text {short_wire_length }} i(l) d l\right)^{m}
$$

Where error_length is the units of gate pitch from which this gate starts to glitch, short_wire_length is the length that we assume the wires in the adver- 
sary path will not exceed (about 20 gate pitches) and $m$ is the number of wires segments in the adversary path.

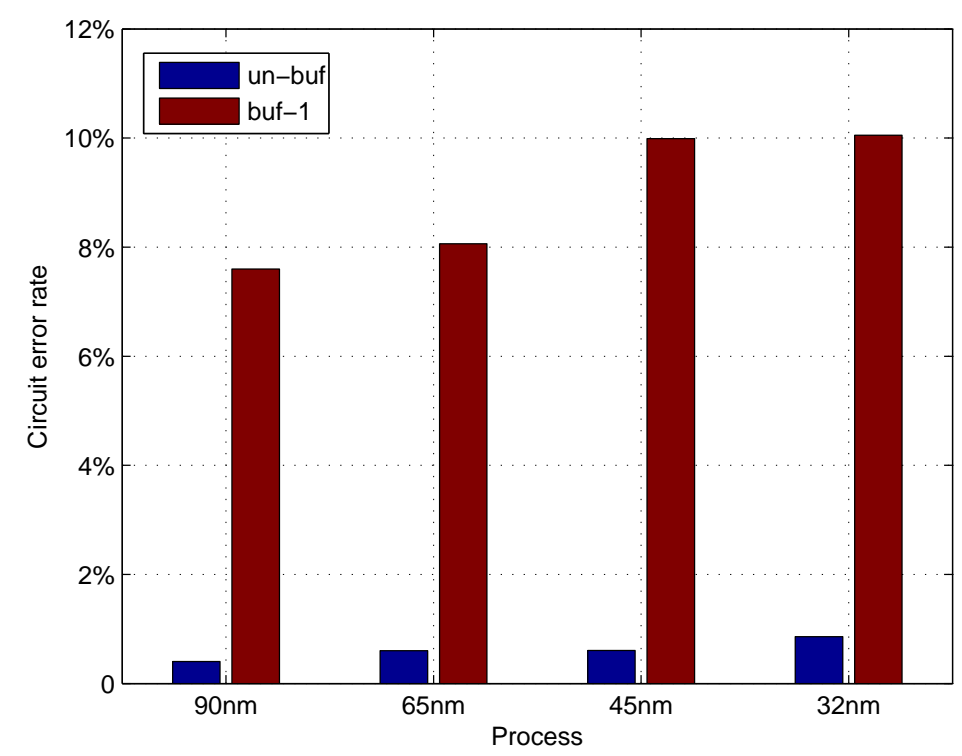

Figure 7.5: The trend of error rate as the technology shrinks -

Figure 7.5 shows the trends of error rate as the process shrinks in one milliongate scale circuit, un-buf indicates the case where buffering is not used on long wires and buf- 1 depicts the error rate when one buffer is inserted into the "direct wire" and no buffers inserted into its "adversary path" (The error rate will increase significantly when a buffer is inserted into the "direct wire". Please refer to the section 4.2.3 for the detailed analysis).

The Figure 7.6 shows the trends of the error rate as the scale increases from 0.5 million to 4 million under the $90 \mathrm{~nm}$ process.

As can be seen from these figures, the error rate increases as the technology shrinks and the buffer insertion technique will increase the error rate significantly. 


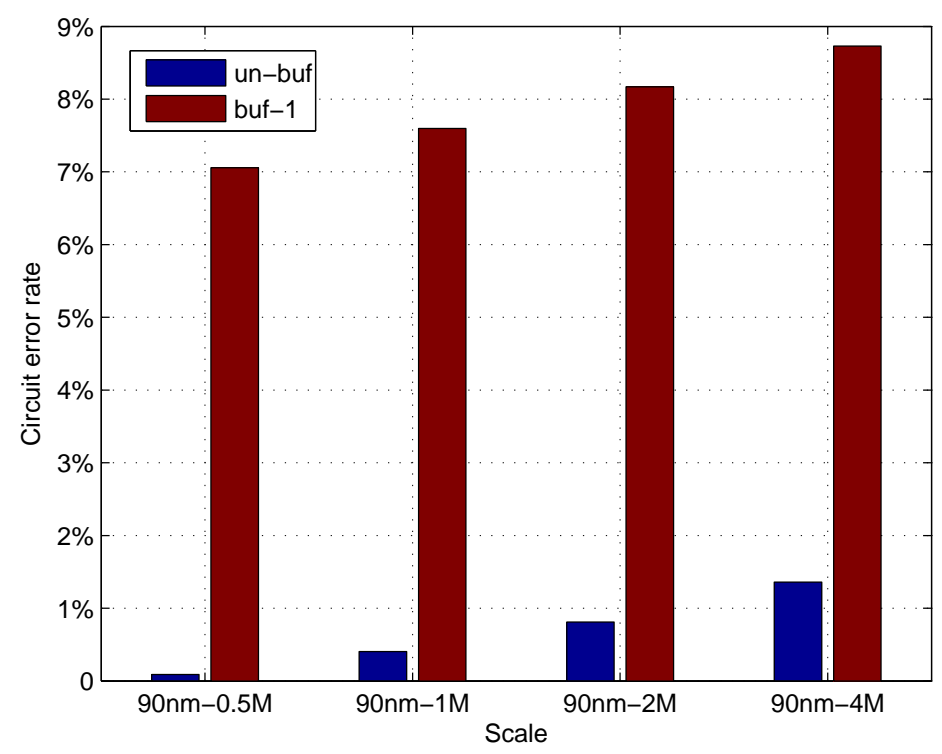

Figure 7.6: The trend of error rate as the scale increases -

Also, the error rate increases remarkably with the scale of circuit. The results suggest that SI circuits will become less safe in the future.

The Figure 7.7 shows the delay penalty to eliminate all the glitches in one million gates scale using different padding methods (buffer and one-direction currentstarved delay). The delays are inserted on wire $w 4$ and $w 14$ to just counter the maximum wire length delay, the environment is assumed to be zero delay and the delay penalty is calculated as the maximum latency increase in the slowest STG cycle. 


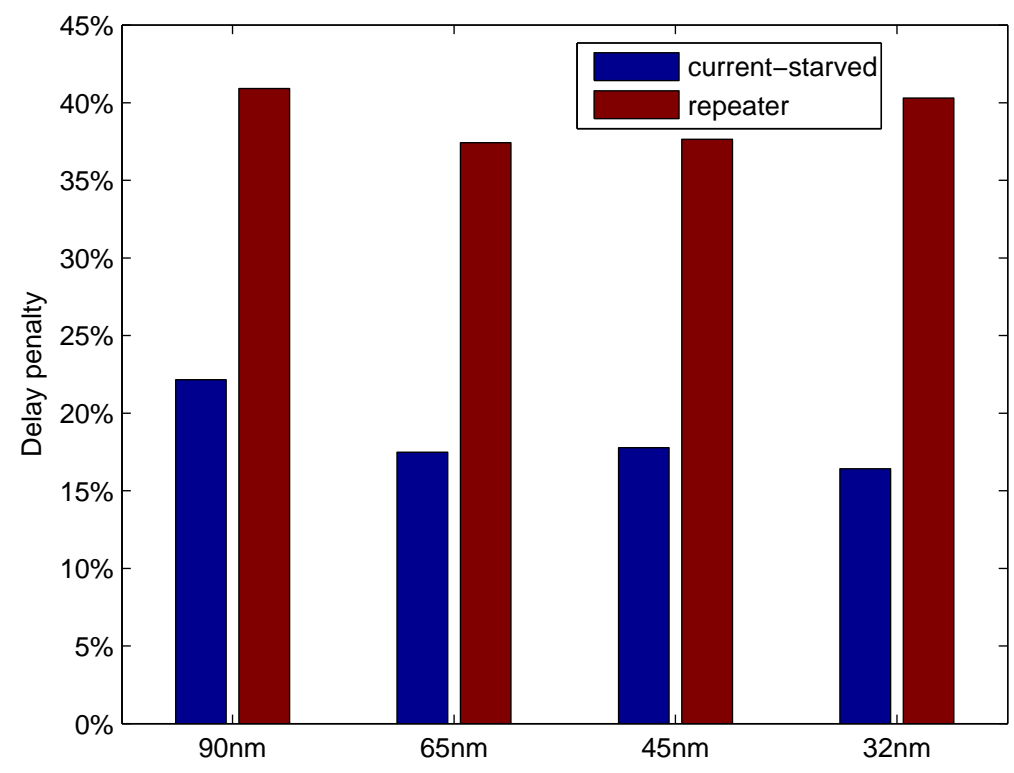

Figure 7.7: The delay penalty -

\subsection{Benchmarks}

This section describes automated tool and presents the benchmark results on a set of popular SI circuits.

\subsubsection{Description of the Tool}

The technique presented in Chapter 5 has been implemented in the tool Check_hazard, which reads an STG as the circuit specification and a restricted EQN file as the circuit description.

The STG to the tool is in the astg format as was used in the tools petrify [60] and sis [69], since the astg format is widely used in the tools related to the synthesis and verification of SI circuits. This format will not be introduced in 
detail here. Currently, the circuit specification format used in the tool is in a restricted $E Q N$ format. In this format, each line contains an equation in the sum of product form to specify the pull-up function of one gate in the circuit. No brackets are allowed. The literals in each product term are connected by the symbol $*$ and different product terms are connected by the symbol + . The negation of a signal is suffixed by "'". An equation is terminated with a ";". The synthesized netlists from some popular tools such as petrify and sis might need to be preprocessed before being input to the tool. For example, for a C-element with the input signal A and the negation of signal B, the equation should be like:

$$
C=A^{*} B^{\prime}+A^{*} C+B^{*} C ;
$$

The equation $C=A^{*} B^{\prime}+C^{*}\left(A+B^{\prime}\right)$; is not correct because it contains a pair of brackets. The example of an STG in the astg format is shown below: 
.model imec-ram-read-sbuf

inputs req precharged prnotin wenin wsldin

.outputs ack wsen prnot wen wsld

.internal $\csc 0$ map0 i0 i2 i4 i8

.graph

req $+\mathrm{i} 4+$

$\mathrm{i} 4+$ prnot +

prnot + prnotin +

precharged + prnot +

prnotin + wen +

wen + precharged- wenin +

precharged- i0-

i0- ack +

wenin $+\mathrm{i} 0-$

ack + req-

req- i8 + wen-

$\mathrm{i} 8+\csc 0-$

wen- wenin-

wsen- wenin-

wenin- wsld $+\mathrm{i} 4-\mathrm{i} 0+$

$\mathrm{i} 0+$ ack-

i4- prnot-

wsld + wsldin + precharged +

wsldin $+\csc 0+$

prnot- prnotin- precharged +

prnotin- i8-

i8- $\csc 0+$

wsld- wsldin-

wsldin- wsen + map0 +

ack- req +

$\mathrm{wsen}+\mathrm{req}+$

$\csc 0+$ wsld- $\mathrm{i} 2$

i2- wsen +

$\csc 0-$ map0-

map0 + ack-

map0- i2+

$\mathrm{i} 2+$ wsen-

.marking $\{<\mathrm{i} 4+$,prnot $+><$ precharged + ,prnot $+>\}$

.end

A circuit specification in the restricted $E Q N$ format is shown below:

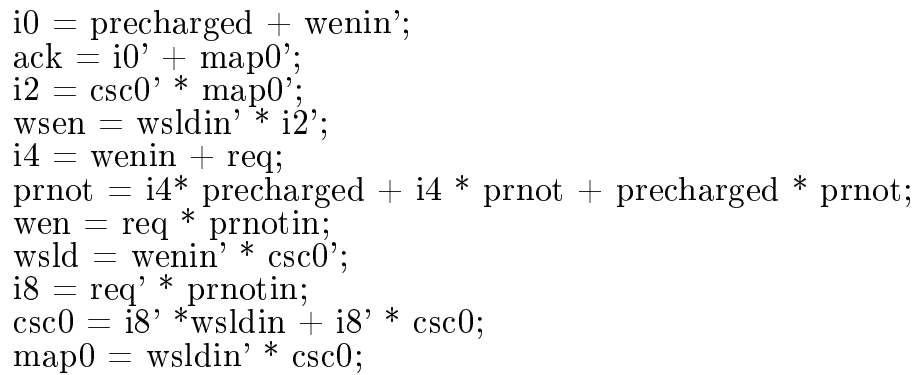

A command line to run the tool is "Check hazard STG.g EQN.eqn". The tool 
will automatically perform the entire process and will report two sets of timing constraints like those in the following block. The first set of timing constraints is the timing constraints to exclude all adversary paths proposed in [55] and the second set is the timing constraints after our relaxation operation.

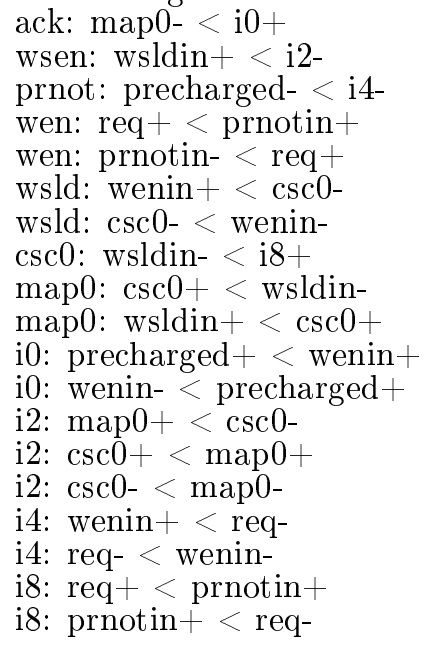

The timing constraints for this circuit to work correctly are: ack: map0- $<\mathrm{i} 0+$

wsen: wsldin $+<\mathrm{i} 2-$

wen: prnotin- $<$ req +

wsld: wenin $+<\operatorname{csc0}-$

csc0: wsldin- $<$ i8-

map0: wsldin $+<\csc 0+$

i0: precharged $+<$ wenin +

i0: wenin- $<$ precharged-

i2: $\operatorname{map} 0+<\csc 0-$

$\mathrm{i} 2: \csc 0+<\operatorname{map0}-$

i4: wenin $+<$ req-

i8: $r e q+<$ prnotin +

The running time for this program is 0.400000 seconds

\subsubsection{Results}

A set of SI circuits is used to test the effects of the proposed method. These specifications are synthesized and then decomposed into multi-level SI circuits 
with each gate containing no more than 4 input signals using the tool petrify [60]. The benchmark results are obtained on a $2.4 \mathrm{Ghz}$ personal computer. The Table 6.2 shows the comparison of generated timing constraints between the proposed method and the timing assumptions proposed in [55], which is currently the weakest formally proved set of conditions.

The column "NO. of adv. before" presents the number of timing constraints before our relaxation process. The timing constraints before our process are equal to the timing constraints to exclude all adversary paths in an SI circuit as is proposed in [55]. The column "NO. of adv. after" presents the number of timing constraints after our relaxation process. The column "NO. of $\leq 5$ level adv. before" presents the number of timing constraints to exclude the adversary paths whose level is less than or equal to 5 (two gates appear in the adversary path) and the column "NO. of $\leq 5$ level adv. after" presents the number of timing constraints in three and five level in our technique. The column "NO. of $\leq 3$ level adv. before" presents the number of timing constraints to exclude the adversary paths whose level is three (one gate appears in the adversary path). The column "NO. of $\leq 3$ level adv. after" presents the number of timing constraints in three level in our technique. As can be seen from this table, the relaxation reduces by around $40 \%$ of unnecessary constraints in all these three aspects. Also, from the table, one could find that the computational time does not increase significantly as the number of states ${ }^{1}$ increases.

\footnotetext{
${ }^{1}$ The number of reachable states shown in this table is the number of states reached by the circuit when the isochronic fork timing assumption is not relaxed. The reachable states would boost significantly when the isochronic fork timing assumption is relaxed.
} 


\begin{tabular}{|c|c|c|c|c|c|c|c|c|c|c|c|}
\hline Name & $\begin{array}{l}\text { NO. of } \\
\text { in }\end{array}$ & $\begin{array}{l}\text { NO. of } \\
\text { out }\end{array}$ & $\begin{array}{l}\text { NO. of } \\
\text { gate }\end{array}$ & $\begin{array}{l}\text { NO. of } \\
\text { states }\end{array}$ & $\begin{array}{l}\text { NO. of adv. } \\
\text { before }\end{array}$ & $\begin{array}{l}\text { NO. of adv. } \\
\text { after }\end{array}$ & $\begin{array}{l}\text { NO. of } \leq \\
5 \text { level adv. } \\
\text { before }\end{array}$ & $\begin{array}{l}\text { NO. of } \leq \\
5 \text { level adv. } \\
\text { after }\end{array}$ & $\begin{array}{l}\text { NO. of } \leq \\
3 \text { level adv. } \\
\text { before }\end{array}$ & $\begin{array}{l}\text { NO. of } \leq \\
3 \text { level adv. } \\
\text { after }\end{array}$ & $\begin{array}{l}\text { CPU } \\
\text { time (s) }\end{array}$ \\
\hline adfast & 3 & 3 & 7 & 94 & 10 & 6 & 4 & 2 & 0 & 0 & 0.04 \\
\hline atod & 3 & 3 & 5 & 28 & 14 & 9 & 10 & 4 & 3 & 1 & 0.01 \\
\hline chu 133 & 3 & 4 & 7 & 41 & 10 & 6 & 2 & 1 & 1 & 1 & 0.04 \\
\hline converta & 2 & 3 & 7 & 36 & 20 & 10 & 10 & 6 & 8 & 4 & 0.1 \\
\hline ebergen & 2 & 3 & 5 & 24 & 10 & 6 & 5 & 5 & 2 & 2 & 0.01 \\
\hline fifo (chu 150) & 3 & 3 & 7 & 64 & 14 & 9 & 5 & 3 & 4 & 2 & 0.05 \\
\hline imec-nak-pa & 4 & 5 & 10 & 110 & 16 & 11 & 8 & 5 & 1 & 1 & 0.24 \\
\hline imec-ram-read-sbuf & 5 & 5 & 11 & 112 & 19 & 12 & 4 & 2 & 2 & 1 & 0.40 \\
\hline imec-sbuf-read-ctl & 2 & 4 & 8 & 32 & 12 & 6 & 3 & 3 & 2 & 2 & 0.06 \\
\hline $\mathrm{mp}$-forward-pkt & 3 & 5 & 8 & 44 & 13 & 8 & 1 & 1 & 0 & 0 & 0.1 \\
\hline nowich & 3 & 3 & 8 & 43 & 10 & 5 & 2 & 1 & 1 & 0 & 0.12 \\
\hline trimos-send & 3 & 6 & 15 & 2023 & 35 & 25 & 15 & 11 & 12 & 8 & 0.12 \\
\hline vbe $5 \mathrm{c}$ & 3 & 6 & 5 & 37 & 8 & 4 & 6 & 1 & 4 & 1 & 0.12 \\
\hline Total ratio & & & & & \multicolumn{2}{|c|}{ after $/$ before $=63.9 \%$} & \multicolumn{2}{|c|}{ after $/$ before $=60.0 \%$} & \multicolumn{2}{|c|}{ after $/$ before $=57.5 \%$} & \\
\hline
\end{tabular}

Table 7.2: Comparison of the timing constraints 


\subsection{Summary}

This chapter first demonstrates the relaxation process of the proposed method using an FIFO controller example. The example is then simulated to study the error rate when the technology develops. Even though the threshold voltage variations are not considered, error rate increases as the semiconductor feature size shrinks. Also, a set of popular SI circuits is used to test the proposed method. The benchmark results suggest that the proposed method excludes around $40 \%$ unnecessary timing constraints compared with the previous research on this issue. 


\section{Chapter 8}

\section{Conclusion and Future Work}

This chapter concludes our work and discusses the possible promotions for the proposed method.

\subsection{Conclusion}

As the semiconductor technology shrinks, process variations become a big obstacle to the circuit design. The synchronous design, which needs to distribute the clock signal throughout the whole circuit, faces severe challenges. The asynchronous design suggests a promising design method for the IC design industry in the coming few decades. The SI design paradigm is more interesting in the variation tolerance aspect compared with other asynchronous design paradigms. Not only because it has the strongest variation tolerance ability for the most specifications, but also because it has comparatively better EDA tool support. However, any asynchronous circuit which is not DI will malfunction if certain 
timing requirements are not met. Any SI circuit should be verified first before implementation.

Current verification methods for SI circuits are mainly focused on checking whether an SI circuit works properly under the isochronous fork timing assumption; that is whether the circuit is functionally conformant to the its specification and is SI. However, as the process shrinks, the isochronic fork timing assumption is no longer reliable. It is required that the timing verification technique should also consider the situation where the isochronic fork assumption is no longer guaranteed. The timing verification is a computationally expensive task. It is especially true for asynchronous circuits which do not have latches to isolate the entire circuit into smaller blocks. This work proposes a technique to derive a set of timing constraints that are sufficient for an SI circuit to work correctly when the isochronic fork timing assumption is relaxed into the intra-operator fork timing assumption. The whole verification task is divided into a set of smaller tasks to avoid exploring the entire reachability space. The high level model (STG) is used to describe the behavior and manipulate the transition causalities in an SI circuit; while, the low level model (SG) is used to check whether hazards would appear in the circuit under a given environment. Each turn, the relaxation process modifies the causality of two ordered transitions into concurrent to include the states that will be reached when the arriving order of two events in the circuit is reversed. Then the SG of the resulting STG will be checked to see whether it contains hazards. If it does, one timing constraint will be generated to guarantee the original ordering of the two events; if it does not, the resulting STG will be accepted which allows the occurrence of the two events in any order. The timing 
assumptions in the resulting STG will have one less adversary path compared with the original one. The generated timing constraints are significantly weaker than the existing proved conditions. Around $40 \%$ unnecessary timing constraints are excluded after local STG relaxation.

The main contributions of the proposed method are:

1) It corrects some wrong conclusions given by previous researchers about the weakest timing assumption in SI circuits.

2) It introduces a hazard checking criterion for SI circuits when the isochronic fork timing assumption is relaxed into the intra-operator fork timing assumption.

3) It proposes a polynomial complexity method for generating a set of timing constraints for an SI circuit to work properly under the intra-operator fork timing assumption. The proposed method checks the hazards of each gate at its local STG. This means that the complexity of hazard checking task is linear to the number of gates in an SI circuit. Also, the complexity of deriving the local STGs for all gates in an SI circuit is polynomial to the number of gate in the circuit if the number of free-choice places in its STG is considered to be a constant.

This work could be improved if the following questions are answered.

\subsection{Future work}

\subsubsection{Non-free-choice place}

The relaxation process in the proposed technique requires that the local STG of the gate must be an MG, where transitions have explicit ordering relations. 
The technique proposed in [8], which could decompose a live and safe free-choice STG into a set of MGs, requires the original STG to be a free-choice one and a guaranteed method of decomposing any STG into a set of equivalent MG's cannot be found in the literature. One solution to this problem is to change a non-free-choice STG into a free-choice STG by writing its SG and then deriving a free-choice STG from this SG using the technique proposed in [49], which has been implemented in the petrify tool. One example is shown in Figure 8.1.

However, the derived free-choice STG might contain too many choice places. The technique proposed in [8] uses brute force to enumerate all possible MG components; so, the number of MGs grows exponentially with respect to the number of free-choice places in the STG. If the STG contains too many choice places, decomposing this STG becomes impractical.

Future research could be carried out to find a technique that could structurally decompose a less restricted class of PN into a set of MGs, thus avoiding introducing too many choice places during transforming the original STG into a free-choice STG.

\subsubsection{Not pure SI circuits}

Many synthesis techniques [70] [71] [72] involve timing assumptions during the logic synthesis to improve the performance. The synthesized circuits are then no longer pure SI circuits. Some timing assumptions involved in these synthesis technique could be expressed by relative timing arcs, for example, the concurrency reduction arcs in [50]. Some timing assumptions could be expressed by decom- 

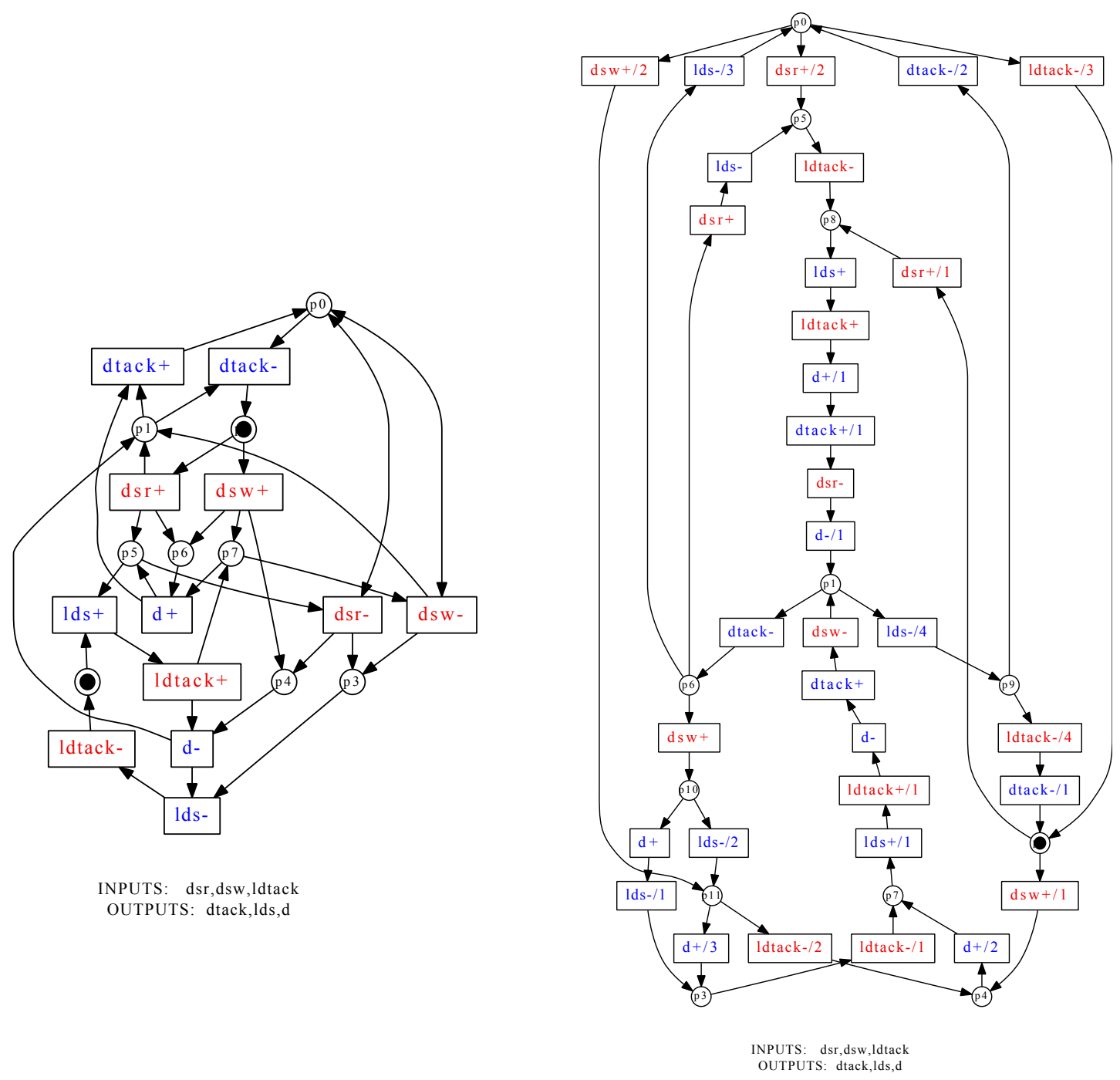

Figure 8.1: A non-free choice STG and its equivalent free-choice STG - 
posing the original STG into a set of component STGs and then using timing arcs to restrict the firing sequences (similar the OR-causality decomposition), like the indistinguishable firing timing assumption in [50]. For those timing assumptions that are not easy to express with timing arcs, one possible approach is to specify a set of states such that these states are not hazardous states even if they are not timing conformant to the function of the gate. 


\section{References}

[1] G. E. MoORE. Cramming more components onto integrated circuits. Electronics Magazine, 1965. 3

[2] M. Donno, E. Macis, And L. Mazzoni. Power-aware clock tree planning. Proceedings of ISPD'04, 2004. 3

[3] V. Tiwari, D. Singh, S. Rajgopal, G. Mehta, R. Patel, And F. BAez. Reducing Power in High-Performance Microprocessors. Proceedings of DAC'98, 2004. 3

[4] M. Favalli and L. Benini. Analysis of glitch power dissipation in CMOS ICs. Proceedings of the 1995 international symposium on Low power design, 2004. 4

[5] ITRS2007. http://www.itrs.net/. 2007. 5

[6] J. Sparso and S. Furber. Principles of Asynchronous Circuit Design: A Systems Perspective. Kluwer Academic Publishers, 2001. 5, 13, 15 
[7] A. J. Martin. The Limitations to Delay-Insensitivity in Asynchronous Circuits. Sixth MIT Conference on Advanced Research in VLSI, 1990. 7

[8] M. HACK. Analysis of production schemata by Petri nets. Technical Report, Massachusetts Institute of Technology Cambridge, 1972. 9, 53, 54, 84,138

[9] L. Lavagno. Synthesis and Testing of Bounded Wire Delay Asynchronous Circuits from Signal Transition Graphs. PhD thesis, U.C. Berkeley, 1992. 11, 30, 89

[10] W. B. Toms And D. A. Edwards. Efficient Synthesis of SpeedIndependent Combinational Logic Circuits. Proceedings of the Asia and South Pacific Design Automation Conference, 2005. 16

[11] Y. Zhou, D. Sokolov, And A. Yakovlev. Cost-aware synthesis of asynchronous circuits based on partial acknowledgement. Proceedings of International Conference on Computer-Aided Design, 2006. 16

[12] A. J. Martin. Asynchronous datapaths and the design of an asynchronous adder. Formal Methods in System Design, 1992. 16

[13] D.A. Huffman. The synthesis of sequential switching circuits. J. Franklin Inst., 1954. 16, 17 
[14] S. H. UnGer. Asynchronous sequential switching circuits. Wiley-interscience, John Wiley \& Sons, Inc., 1969. 17

[15] S. M. NOWICK. Automatic synthesis of burst-mode asynchronous controllers. Technical Report, CSL-TR-95-686, Stanford University, 1995. 17

[16] Robert M. Fuhrer, Niraj K. Jha, Bill Lin, Luis Plana, And et AL. MINIMALIST: An Environment for the Synthesis, Verification and Testability of Burst-Mode Asynchronous Machines, 1999. 18

[17] K. Y. Yun AND D. L. Dill. Unifying Synchronous/Asynchronous State Machine Synthesis. Proceedings of the IEEE/ACM International Conference on Computer-Aided Design, 1993. 18

[18] K. Y. Yun And D. L. Dill. Automatic synthesis of extended burstmode circuits. I. (Specification and hazard-free implementations). $\underline{\text { IEEE Transactions on Computer-Aided Design of Integrated Circuits and }}$ Systems, 1999. 18

[19] K. Y. Yun and D. L. Dill. Automatic synthesis of extended burstmode circuits: part II (automatic synthesis). IEEE Transactions on Computer-Aided Design of Integrated Circuits and Systems, 1999. 18

[20] D. E. Muller And W. S. BartKy. A theory of asynchronous circuits. Proceedings of an International Symposium on the Theory of Switching, 1959. 19 
[21] D. E. Muller. Asynchronous logics and application to information processing. Proceedings of the Symposium on the Application of Switching Theory in Space Technology, 1963. 19

[22] A. J. MARTIN. The design of a delay-insensitive microprocessor: an example of circuit synthesis by program transformation. Hardware Specification, Verification and Synthesis: Mathematical Aspects, 1989. 19, 38

[23] J. A. Brzozowski and J. Ebergen. On the Delay-Sensitivity of Gate Networks. IEEE Transactions on Computers, 1992. 19

[24] R. Manohar and A. J. Martin. Quasi-delay-insensitive circuits are Turing-complete. Second International Symposium on Advanced Research in Asynchronous Circuits and Systems, 1995. 20

[25] A. J. Martin. Programming in VLSI: From Communicating Processes to Delay-Insensitive Circuits. Developments in Concurrency and Communication, 1990. 20

[26] S. H. Unger. Hazards, Critical Races, and Metastability. IEEE Transactions on Computers, 1995. 20

[27] K. S. Stevens, S. Rotem, R. Ginosar, P. Beerel, C. J. Myers, K. Y. Yun, R. Kol, C. Dike, and M. Roncken. An Asynchronous Instruction Length Decoder. IEEE Journal of solid-state circuits, 2001. 24 
[28] A. Davis, B. Coates, and K. Stevens. The Post Office experience: Designing a large asynchronous chip. In Proceeding of the Twenty-Sixth Hawaii International Conference on System Sciences, 1993. 24

[29] P. A. Beerel and T.H.-Y. Meng. Automatic Gate-Level Synthesis of Speed-Independent Circuits. In Proceeding of IEEE/ACM International Conference on Computer-Aided Design, 1992. 24

[30] E. Pastor, J. Cortadella, A. Kondratyev, and O. Roig. Structural methods for the synthesis of speed-independent circuits. IEEE Transactions on Computer-Aided Design, 1998. 24

[31] D. Edwards and A. Bardsley. Balsa: An Asynchronous Hardware Synthesis Language. The Computer Journal, 2002. 24

[32] D. Sokolov, A. Bystrov, And A. Yakovlev. Direct Mapping of Low-Latency Asynchronous Controllers From STGs. Computer-Aided Design of Integrated Circuits and Systems, IEEE Transactions on, 2007. 24

[33] D. L. Shang, F. Burns, A. Koelmans, A. Yakovlev, and F. Xia. Asynchronous system synthesis based on direct mapping using VHDL and Petri nets. IEE Proceedings - Computers and Digital Techniques, 2004. 24

[34] F. P. Burns, D. L. Shang, A. Koelmans, and A. Yakovlev. An Asynchronous Synthesis Toolset Using Verilog. Design, Automation and Test in Europe Conference and Exposition, 2004. 24 
[35] S. M. Burns. General Conditions for the Decomposition of State Holding Elements. Asynchronous Circuits and Systems, IEEE International Symposium on, 1996. 24

[36] A. Kondratyev, M. Kishinevsky, J. Cortadella, L. Lavagno, And Alex Yakovlev. Technology mapping for speed-independent circuits: decomposition and resynthesis. In Proceeding of International $\underline{\text { Symposium on Advanced Research in Asynchronous Circuits and Systems, }}$ 1997. 24

[37] A. Kondratyev, J. Cortadella, M. Kishinevsky, L. Lavagno, and Alexander YAKOVlev. Logic decomposition of speed-independent circuits. In Proceedings of the IEEE, 1999. 24

[38] V. Khomenko. Model Checking Based on Prefixes of Petri Net Unfoldings. PhD thesis, University of Newcastle upon Tyne, 2003. 24, 49

[39] O. Roig, J. Cortadella, and E. Pastor. Hierarchical gate-level verification of speed-independent circuits. Proceeding of Second Working Conference on Asynchronous Design Methodologies, 1995. 24, 49, 51

[40] I. Poliakov, A. Mokhov, A. Rafiev, D. Sokolov, and A. Yakovlev. Automated verification of asynchronous circuits using circuit Petri nets. Proceedings of the 14th IEEE International Symposium on Asynchronous Circuits and Systems, 2008. 24, 49, 51 
[41] D. L. Dill. Trace Theory for Automatic Hierarchical Verification of Speed-independent Circuits. MIT Press, 1989. 24

[42] P. A. Beerel, J. Burch, and T. H. Y. Meng. Efficient verification of determinate speed-independent circuits. Proceedings of the 1993

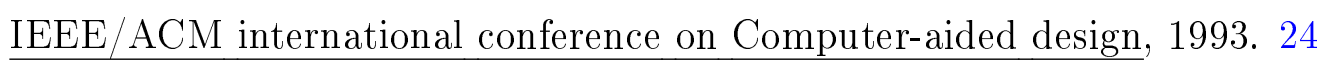

[43] O. Roig, J. Cortadella, M. A. Pena, and E. Pastor. Automatic generation of synchronous test patterns for asynchronous circuits. In Proceeding of ACM/IEEE Design Automation Conference, 1997. 24

[44] C. A. Petri. Kommunikation mit automaten. PhD thesis, University of Bonn, 1962. 25

[45] T. Murata. Petri nets: Properties, analysis and applications. PROCEEDINGS OF THE IEEE, 1989. 27

[46] J. Desel and J. Esparza. Free choice Petri nets. Cambridge University Press, 1995. 27

[47] L. Rosenblum And A. Yakovlev. Signal graphs: From self-timed to timed ones. Proceedings of International Workshop on Timed Petri Nets, 1985. 28,30

[48] T. A. CHU. Synthesis of self-timed VLSI circuits from graphtheoretic specifications. $\mathrm{PhD}$ thesis, MIT Laboratory for Computer Science, 1987. 28, 31, 84 
[49] J. Cortadella, M. Kishinevsky, L. Lavagno, and A. Yakovlev. Deriving Petri nets from finite transition systems. Computers, IEEE Transactions on, 1998. 29, 138

[50] J. Cortadella, M. Kishinevsky, A. Kondratyev, L. Lavagno, And A. Yakovlev. Logic Synthesis of Asynchronous Controllers and Interfaces. Springer-Verlag, 2002. 31, 35, 36, 138, 140

[51] K. Van Berkel, F. Huberts, and A. Peeters. Stretching quasi delay insensitivity by means of extended isochronic forks. Proceedings of ASYNC'95, 1995. 35

[52] C. Piguet. Supplementary condition for STG-designed speedindependent circuits. Electronics Letters, April 1998. 35, 36

[53] KeES VAn Berkel. Beware the Isochronic Fork. INTEGRATION, The VLSI Journal, 1992. 35, 37

[54] N. Sretasereekul and T. NANya. Eliminating isochronic-fork constraints in quasi-delay-insensitive circuits. Proceedings of the 2001 Asia and South Pacific Design Automation Conference, 2001. 35, 41, 49

[55] S. Keller, M. Katelman, And A. J. Martin. A Necessary and Sufficient Timing Assumption for Speed-Independent Circuits. Proceedings of Asynchronous Circuits and Systems, International Symposium on, 2009, 2009. 35, 42, 45, 46, 50, 59, 131, 132 
[56] C. Piguet. Logic synthesis of race-free asynchronous CMOS circuits. IEEE J. Solid-State Circuits, March 1991. 37

[57] N. Starodoubtsev and S. Bystrov. Behavior and Synthesis of Two-Input Gate Asynchronous Circuits. Proceedings of the 11th IEEE international Symposium on Asynchronous Circuits and Systems, March 2005. 37

[58] L. Lin And W. Burleson. Analysis and mitigation of process variation impacts on Power-Attack Tolerance. Proceedings of Proceedings of the 46th Annual Design Automation Conference, 2009. 38

[59] H. Saito, A. Kondratyev, J. Cortadella, L. Lavagno, And A. YAKOVLEV. What is the cost of Delay Insensitivity. Proceedings of the 1999 IEEE/ACM international conference on Computer-aided design, 1999. 49

[60] J. Cortadella, M. Kishinevsky, A. Kondratyev, L. Lavagno, And A. Yakovlev. Petrify: A Tool for Manipulating Concurrent Specifications and Synthesis of Asynchronous Controllers. IEICE TRANSACTIONS on Information and Systems, 1996. 52, 128, 132

[61] M. Schafer, W. Vogler, and P. Jancar. Determinate STG Decomposition of Marked Graphs. Applications and Theory of Petri Nets 2005, 26th International Conference, 2005. 68, 69, 71

[62] M. Diaz. Petri Nets: Fundamental Models, Verification and Applications. Wiley-ISTE, 2009. 69 
[63] A. Yakovlev, M. Kishinevsky, A. Kondratyev, and L. Lavagno. OR causality: Modelling and hardware implementation. Applications and Theory of Petri Nets 1994, International Conference, 1994. 75,77

[64] A. Yakovlev, M. Kishinevsky, A. Kondratyev, L. Lavagno, And M. Pietkiewicz-Koutny. On the models for asynchronous circuit behaviour with OR causality. Formal Methods in System Design, 1996. 75,77

[65] M. G. Johnson And E. L. Hudson. A variable delay line PLL for CPU-coprocessor synchronization. IEEE Journal of Solid-State Circuits, 1988. 123

[66] M. Maymandi-Nejad and M. Sachdev. A Digitally Programmable Delay Element: Design and Analysis. IEEE TRANSACTIONS ON VERY LARGE SCALE INTEGRATION SYSTEMS, 2003. 123

[67] http://ptm.asu.edu/. 124

[68] J. A. Davis, V. K. De, And J. D. Meindl. A stochastic wire-length distribution for gigascale integration (GSI). IEEE Transactions on Electron Devices, 1998. 124

[69] E. M. Sentovich, K. J. Singh, L. Lavagno, C. Moon, R. Murgai, A. Saldanha, H. Savoj, P. R. Stephan, R. K. Brayton, and A. Sangiovanni-vincentelli. SIS: A System for Sequential Circuit Synthesis. technique report, 1992. 128 
[70] J. Cortadella, M. Kishinevsky, S. M. Burns, A. Kondratyev, L. Lavagno, K. S. Stevens, A. Taubin, and A. Yakovlev. Lazy Transition Systems and Asynchronous Circuit Synthesis with Relative Timing Assumptions. In IEEE Transactions on CAD, 2002. 138

[71] K. S. Stevens, S. Rotem, S. M. Burns, J. Cortadella, R. Ginosar, M. Kishinevsky, and M. Roncken. CAD Directions for High Performance Asynchronous Circuits. In Proceedings of the Digital Automation Conference, 1999. 138

[72] P. Vanbekbergen, G. Goossens, and B. Lin. Modeling and Synthesis of Timed Asynchronous Circuits. In Proceedings of the European Design Automation Conference, 1994. 138 


\section{Index}

adversary path, 43

adversary path timing assumption, 43

input state, 11

AND-causality, 90

autonomous circuits, 14

burst mode, 15

circuit, 14

clause, 12

cover, 12

irredundant prime cover, 12

off-set cover, 12

on-set cover, 12

prime cover, 12

redundant cover, 12

cube, 12

delay, 13

bounded delay, 13

inertial delay, 13

pure delay, 13

unbounded delay, 13

delay-insensitive, 19

environment, 14

fundamental mode, 15

gate, 11

glitch, 20

Huffman style, 16

implicant, 12

prime implicant, 12

input-output mode, 15

inter-operator fork, 41

intra-operator fork, 41

isochronic fork, 23

literal, 12

logic function, 11

marking

marking set, 26

Muller style, 18

off-set, 11

on-set, 11

OR-causality, 80, 90

Petri net, 25

live, 27

marked graph, 27

marking, 25

MG component, 52

place, 25

choice place, 27

implicit, 67

loop-only place, 68

merge place, 27

shortcut place, 68

safe, 27

transition, 25

concurrent, 27

conflict, 27

enabled transition, 26 
predecessor transition, 28

successor transition, 28

race hazard, 5

SG, 30

excitation region, 31

excited, 31

quiescent region, 32

stable, 31

SI

acknowledgement, 34

signal, 14

speed-independent, 20, 23

STG, 28

arc, 67

redundant, 67

consistency, 29

implementation STG, 29, 51

specification STG, 29, 51

timing conformance, 72 